\title{
Transition-metal Controlled Diastereodivergent Radical Cyclization/Azidation Cascade of 1,7-Enynes
}

\section{Supporting Information}

Yingying Zhao, ${ }^{\dagger}$ Yancheng $\mathrm{Hu},{ }^{\dagger}$ Haolong Wang, Xincheng $\mathrm{Li}^{*}$ and Boshun Wan*

Dalian Institute of Chemical Physics, Chinese Academy of Sciences, 457 Zhongshan Road, Dalian 116023, China

${ }^{\dagger}$ These authors contributed equally to this work.

*E-mail: xcli@dicp.ac.cn; bswan@dicp.ac.cn

\section{Table of Contents}

1. Optimization of reaction conditions.....................................S1

2. X-ray structure of trans-2a ....................................................S2

3. Mechanistic studies................................................................. 3

4. Copies of NMR spectroscopy ..............................................S8 


\section{Optimization of reaction conditions ${ }^{[a]}$}

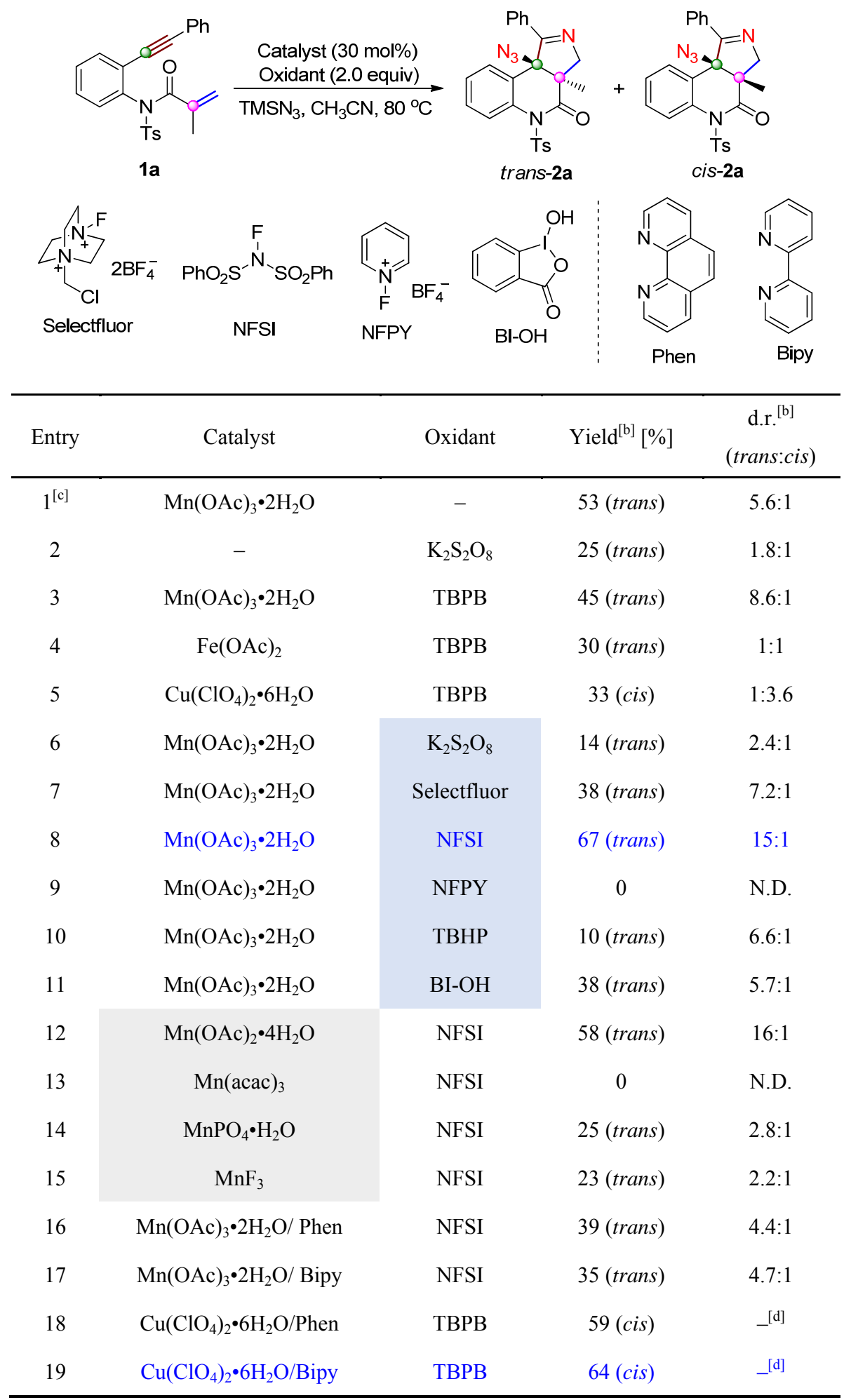

[a] Reaction conditions: $1 \mathrm{a}(0.10 \mathrm{mmol}), \mathrm{Mn}(\mathrm{OAc})_{3} \cdot 2 \mathrm{H}_{2} \mathrm{O}(30 \mathrm{~mol} \%)$, ligand (33 mol\% when added), $\mathrm{TMSN}_{3}$ (6.0 equiv), and oxidant (2.0 equiv) in $\mathrm{CH}_{3} \mathrm{CN}(2.0 \mathrm{~mL})$ at $80{ }^{\circ} \mathrm{C}$ for $22 \mathrm{~h}$. [b] Determined by HPLC analysis of the crude reaction mixture before work-up. Naphthalene was 
used as the internal standard. [c] $\mathrm{Mn}(\mathrm{OAc})_{3} \cdot 2 \mathrm{H}_{2} \mathrm{O}$ (3.0 equiv) was employed. [d] cis-2a was observed as the single isomer. N.D. $=$ No detected. TBPB $=$ tert-Butyl peroxybenzoate. TBHP $=$ tert-Butyl hydroperoxide.

\section{X-ray structure of trans-2a}

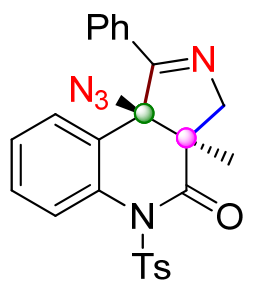

trans-2a

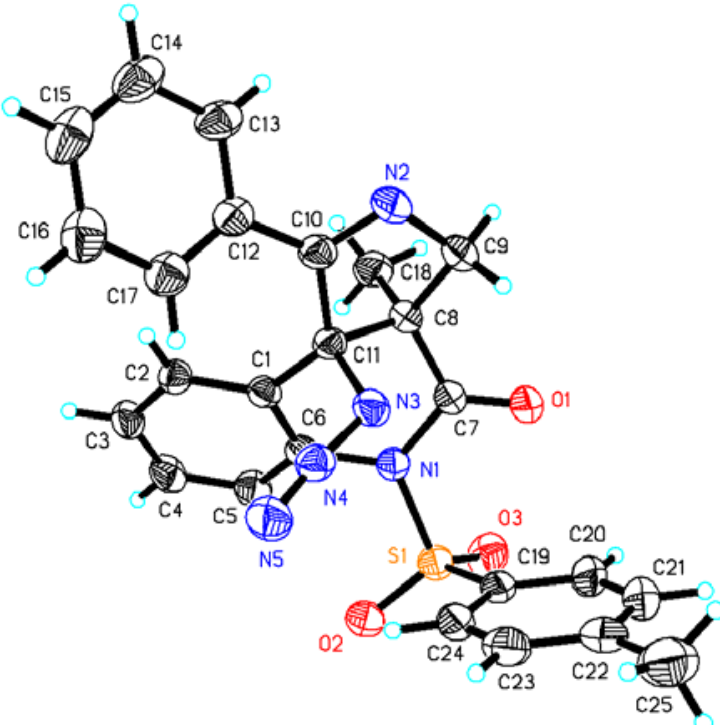

The ellipsoid contour percent probability level is 50\%. CCDC 1449869 (trans-2a) contains the supplementary crystallographic data for this paper. These data can be obtained free of charge from The Cambridge Crystallographic Data Centre via www.ccdc.cam.ac.uk/data_request/cif.

Crystal data and structure refinement for cd15107.

Identification code

Empirical formula

Formula weight

Temperature

Wavelength

Crystal system

Space group

Unit cell dimensions

Volume

Z

Density (calculated) cd15107

$\mathrm{C} 25 \mathrm{H} 21 \mathrm{~N} 5 \mathrm{O} 3 \mathrm{~S}$

471.53

293(2) K

$0.71073 \AA$

Triclinic

P -1

$\mathrm{a}=9.149(5) \AA \quad=80.924(13)^{\circ}$.

$\mathrm{b}=10.448(6) \AA \quad=71.346(11)^{\circ}$

$\mathrm{c}=12.712(8) \AA$

$=89.687(13)^{\circ}$.

\section{$1135.6(12) \AA^{3}$}

2 $1.379 \mathrm{Mg} / \mathrm{m}^{3}$ 


\begin{tabular}{ll}
\hline Absorption coefficient & $0.181 \mathrm{~mm}^{-1}$ \\
$\mathrm{~F}(000)$ & 492 \\
Crystal size & $0.230 \times 0.180 \times 0.120 \mathrm{~mm}^{3}$ \\
Theta range for data collection & 1.976 to $25.498^{\circ}$. \\
Index ranges & $-11<=\mathrm{h}<=11,-10<=\mathrm{k}<=12,-11<=1<=15$ \\
Reflections collected & 6552 \\
Independent reflections & $4212[\mathrm{R}(\mathrm{int})=0.0234]$ \\
Completeness to theta $=25.242^{\circ}$ & $99.6 \%$ \\
Absorption correction & $\mathrm{Semi}-\mathrm{empirical}$ from equivalents \\
Max. and min. transmission & 0.7456 and 0.6379 \\
Refinement method & Full-matrix least-squares on $\mathrm{F}^{2}$ \\
Data / restraints / parameters & $4212 / 0 / 309$ \\
Goodness-of-fit on $\mathrm{F}^{2}$ & 1.026 \\
Final R indices [I $>2$ sigma(I)] & $\mathrm{R} 1=0.0483, \mathrm{wR} 2=0.1241$ \\
$\mathrm{R}$ indices (all data) & $\mathrm{R} 1=0.0616, \mathrm{wR} 2=0.1346$ \\
Extinction coefficient & $\mathrm{n} / \mathrm{a}$ \\
Largest diff. peak and hole & 0.264 and $-0.257 \mathrm{e} . \AA^{-3}$ \\
\hline
\end{tabular}

\section{Mechanistic studies}

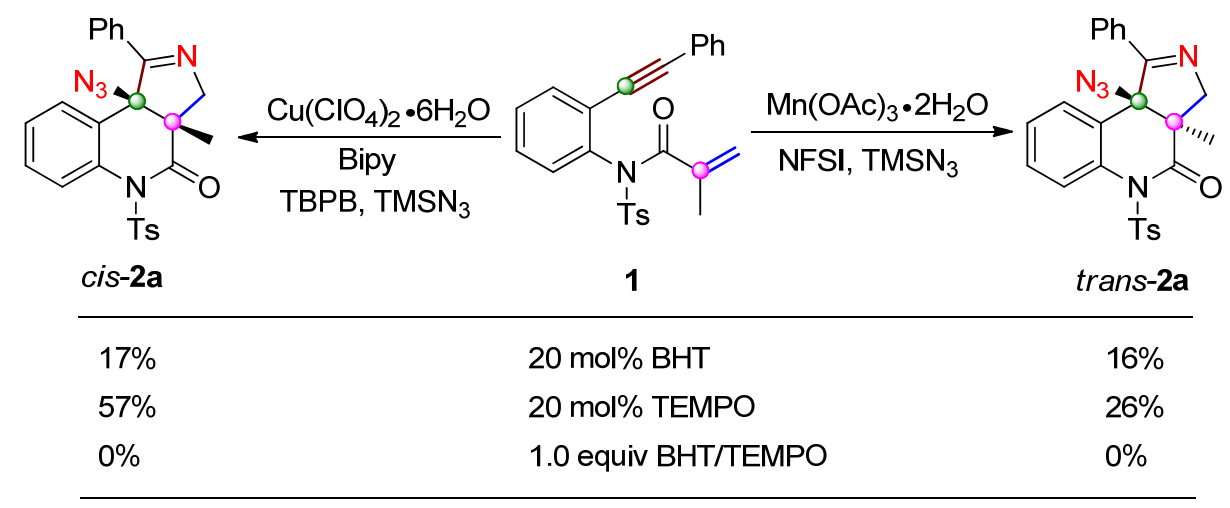

Submitting 1.0 equivalent of TEMPO or BHT to the standard conditions gave no desired products, revealing that a radical-mediated pathway is involved in both reactions. 


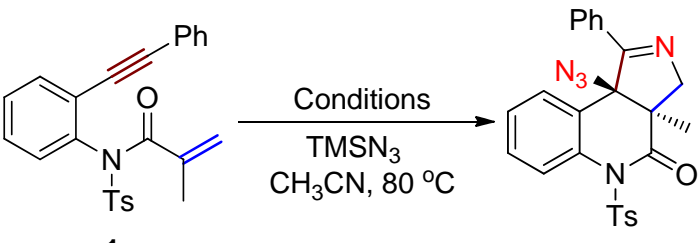

$1 a$

trans-2a

\begin{tabular}{|c|c|c|}
\hline Entry & Conditions & Comments \\
\hline 1 & $\begin{array}{l}\text { 1) } \mathrm{Mn}(\mathrm{OAc})_{3} \cdot 2 \mathrm{H}_{2} \mathrm{O}(30 \mathrm{~mol} \%), 22 \mathrm{~h} \\
\text { 2) } \mathrm{NFSI}(2 \text { equiv) }\end{array}$ & $\begin{array}{l}\text { In the absence of NFSI, } \\
\text { trans-2a }(3 \%) \\
\text { After } 22 \text { h, NFSI was added, } \\
\text { trans-2a }(44 \%) \text {, d.r. (8.7:1) }\end{array}$ \\
\hline 2 & $\begin{array}{l}\text { 1) } \mathrm{Mn}(\mathrm{OAc})_{2} \cdot 4 \mathrm{H}_{2} \mathrm{O}(30 \mathrm{~mol} \%), 22 \mathrm{~h} \\
\text { 2) } \mathrm{NFSI}(2 \text { equiv) }\end{array}$ & $\begin{array}{l}\text { In the absence of NFSI, } \\
\text { trans-2a (0\%) } \\
\text { After } 22 \text { h, NFSI was added, } \\
\text { trans-2a (50\%), d.r. (11.6:1) }\end{array}$ \\
\hline 3 & NFSI (2 equiv) & $\begin{array}{l}\text { In the absence of } \mathrm{Mn}(\mathrm{III}) \\
\text { trans-2a }(16 \%), \text { d.r. }(1.5: 1)\end{array}$ \\
\hline
\end{tabular}

As shown in entry 1, it is suggested that $\mathrm{Mn}(\mathrm{III})$ is employed as azidyl radical initiator and NFSI oxidizes in situ formed $\mathrm{Mn}$ (II) to regenerate the catalytic cycle. Mn(II) cannot promote the reaction, whereas after NFSI was added, trans-2a was readily obtained, which indicated that $\mathrm{Mn}(\mathrm{II})$ is first oxidized to $\mathrm{Mn}(\mathrm{III})$ and then $\mathrm{Mn}(\mathrm{III})$ initiate the process. In the absence of manganese, NFSI only gave poor yield and selectivity, demonstrating $\mathrm{Mn}$ is involved in the d.r.-determining step.

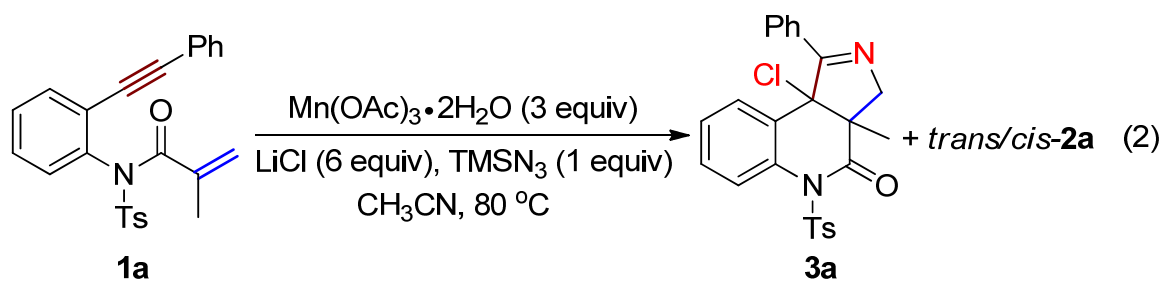

$[\mathrm{M}+\mathrm{H}]^{+}: 465.1034$

To a sealed tube were added enynes $1 \mathrm{a}(0.2 \mathrm{mmol}), \mathrm{Mn}(\mathrm{OAc})_{3} \cdot 2 \mathrm{H}_{2} \mathrm{O}$ (3.0 equiv.), $\mathrm{LiCl}$ (6.0 equiv.), and $\mathrm{MeCN}(4 \mathrm{~mL})$ in sequence. Subsequently $\mathrm{TMSN}_{3}$ (1.0 equiv.) was introduced and then stirred at $80{ }^{\circ} \mathrm{C}$ for $22 \mathrm{~h}$. Upon completion, the solvent was removed and purification by flash chromatography on silica gel (petroleum ether/EtOAc: 10/1) afforded trans-2a and a mixture of cis-2a and 3a. The structure of 3a was confirmed by NMR and HRMS. This result explicitly illustrated that $\mathrm{Mn}(\mathrm{III})-\mathrm{Cl}$ complex is formed and then undergoes chloro-ligand transfer oxidation process to deliver 3a. As a consequence, we believe that the in situ formed $L_{n} M-N_{3}$ 
species is involved in the d.r.-determining step, thereby leading to switchable diastereoselectivity.

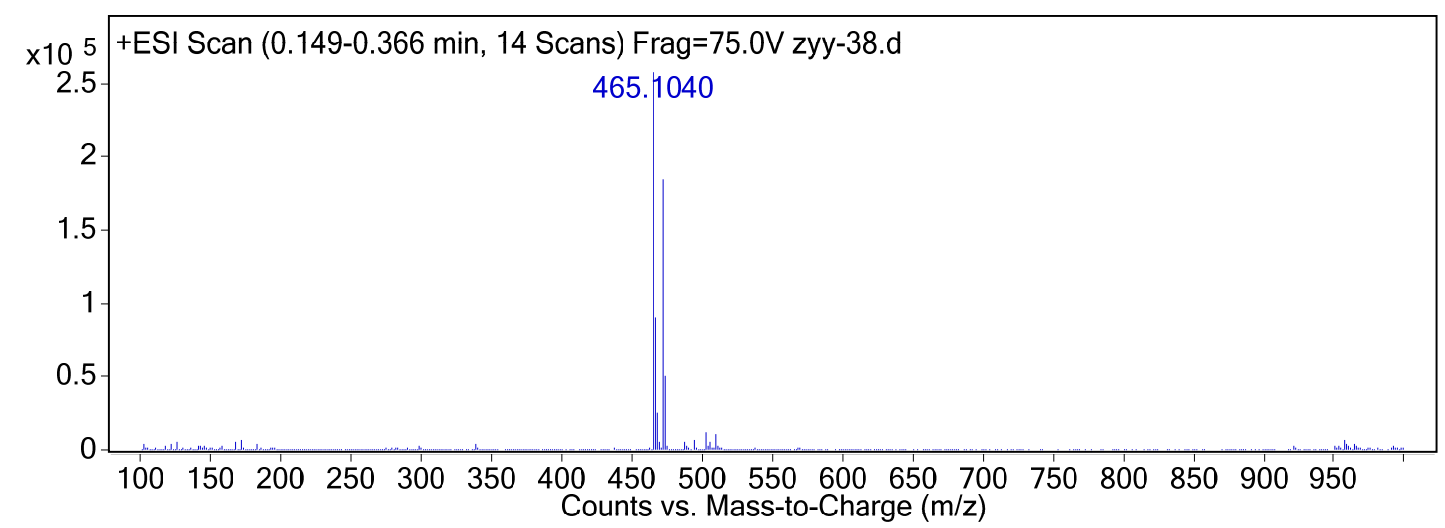

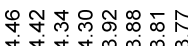

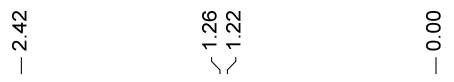

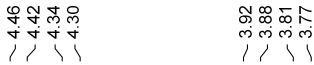
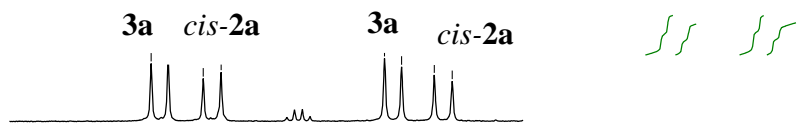

$\begin{array}{llllllllllll}4.7 & 4.6 & 4.5 & 4.4 & 4.3 & 4.2 & 4.1 & 4.0 & 3.9 & 3.8 & 3.7\end{array}$

Mixture of 3a and cis-2a

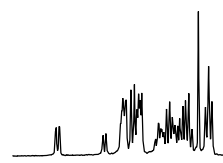

ilit. bili

$\int$
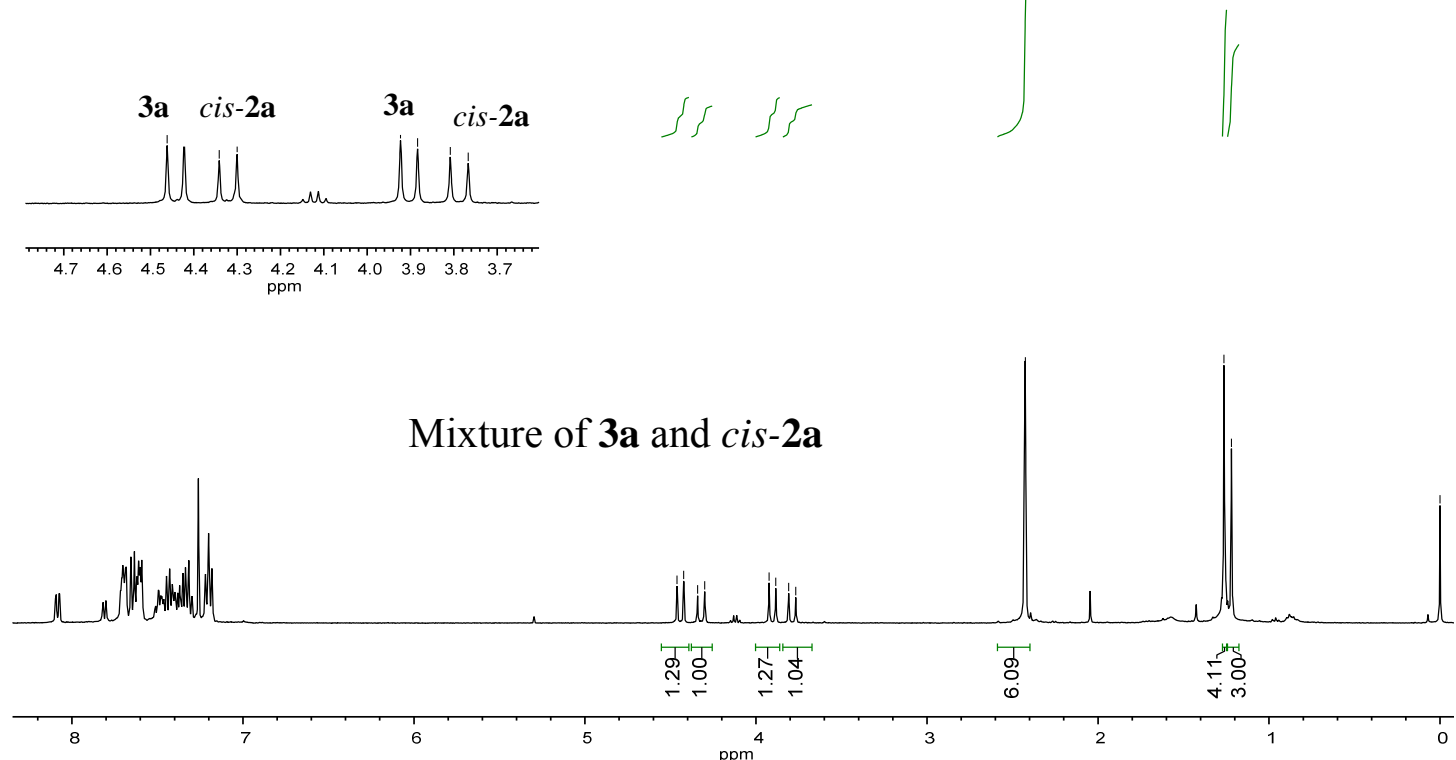


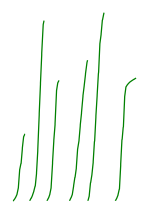

cis-2a

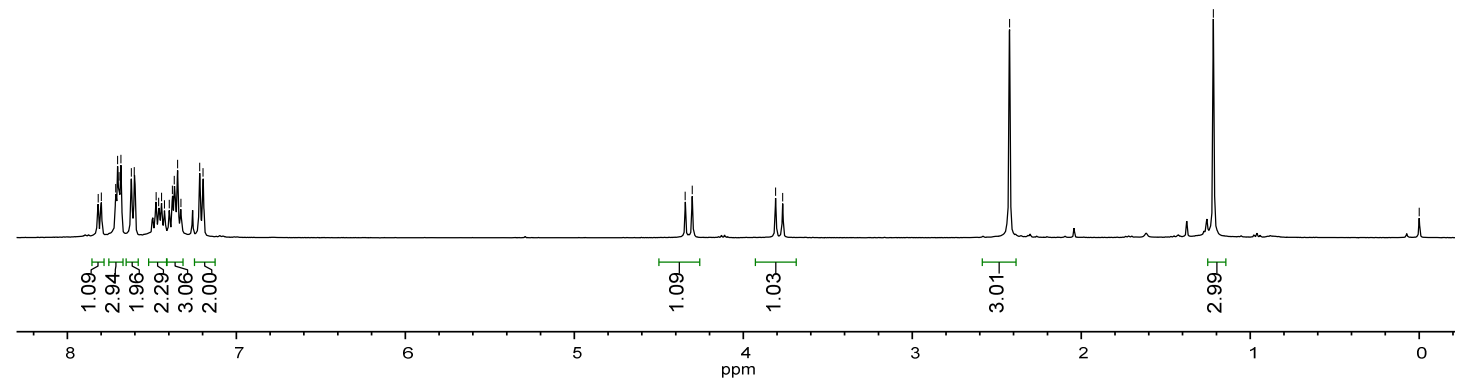

A plausible mechanism is depicted as follows:

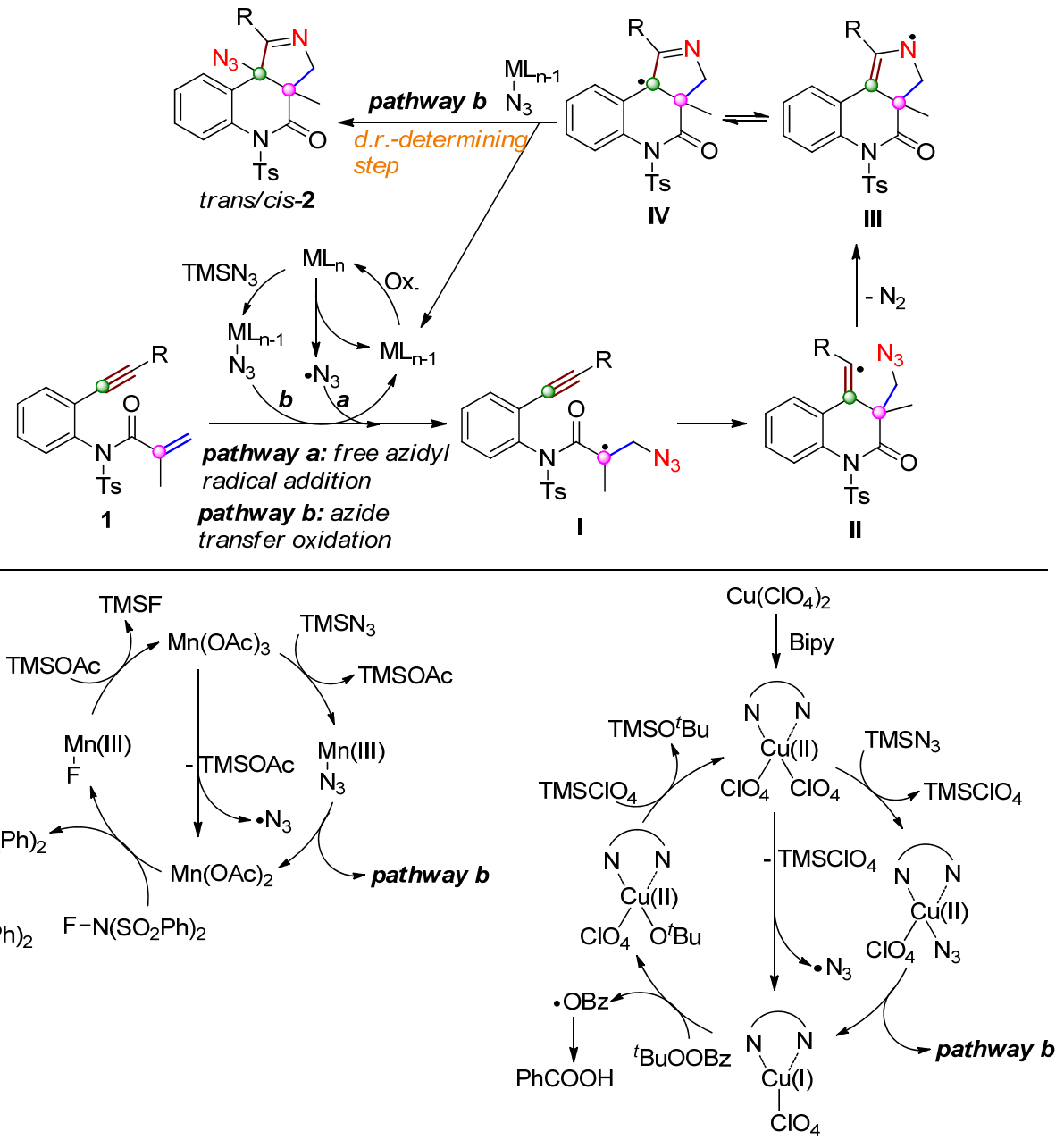


Initially, $\mathrm{TMSN}_{3}$ is oxidized by high-valent transition-metal catalyst to produce a free azidyl radical, which then attacks the alkene moiety of enyne 1, giving alkyl radical I (pathway a). It is noteworthy that direct azide transfer from metal azide complex to alkene cannot be ruled out (pathway b). Radical-triggered 6-exo-dig cyclization of I affords vinyl radical II, which can be further trapped by azidyl group, followed by releasing one molecule of nitrogen to generate aminyl radical III. The tautomer of III, alkyl radical IV, undergoes inner-sphere azide transfer oxidation process, rather than free azidyl radical pathway, to deliver the desired product 2 . The oxidation of low-valent metals $\mathrm{Mn}(\mathrm{II})$ and $\mathrm{Cu}(\mathrm{I})$ by NFSI and TBPB regenerates the catalysts.

Although we have no direct rationale on the coordination modes between transition-metal complex and the substrate, a tentative model for the diastereodivergence was depicted. In the case of $\mathrm{Mn}$-mediated reaction, $\mathrm{Mn}-\mathrm{N}_{3}$ possibly also coordinates with the nitrogen atoms of intermediate IV, following azide transfer to produce trans-2. When $\mathrm{Cu}$ was introduced to the reaction, the pronounced steric hinderance of phenyl group enables the azide attack on the opposite side, thus providing cis-2.

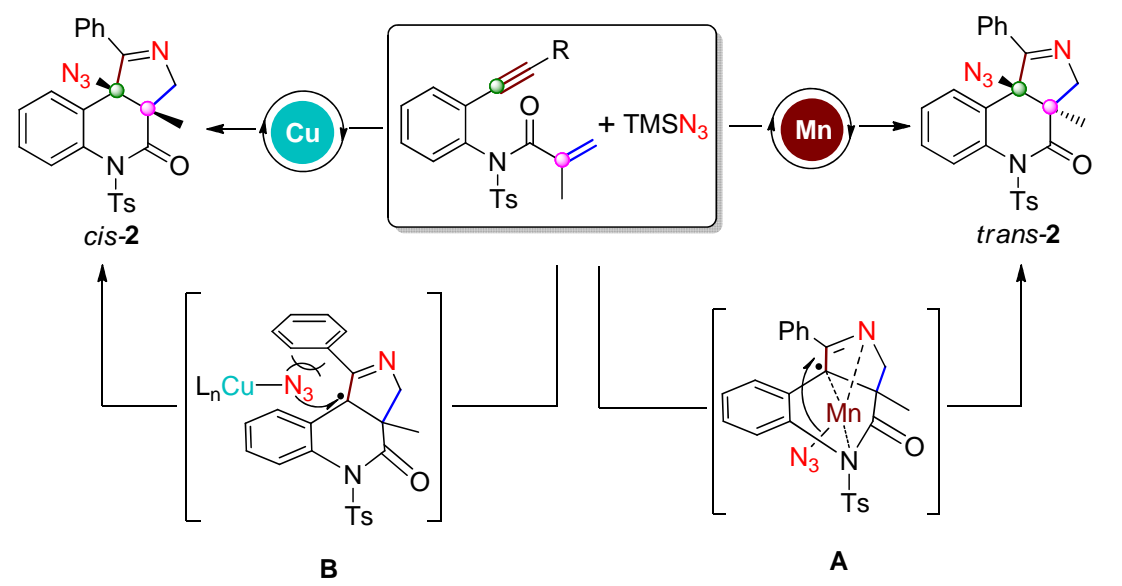




\section{Copies of NMR spectroscopy}

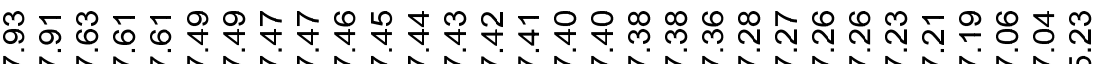
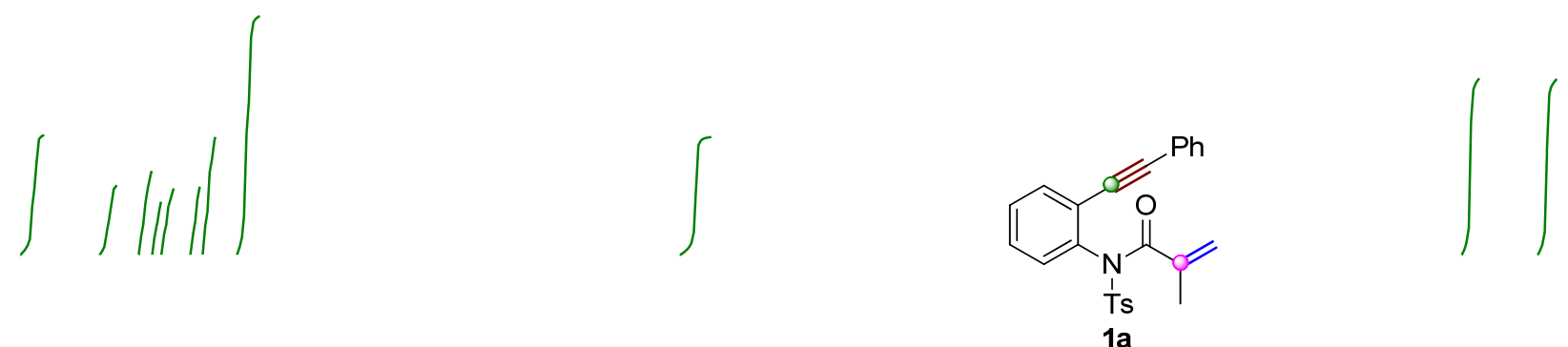

${ }^{1} \mathrm{H} \mathrm{NMR}\left(\mathrm{CDCl}_{3}, 400 \mathrm{MHz}\right)$

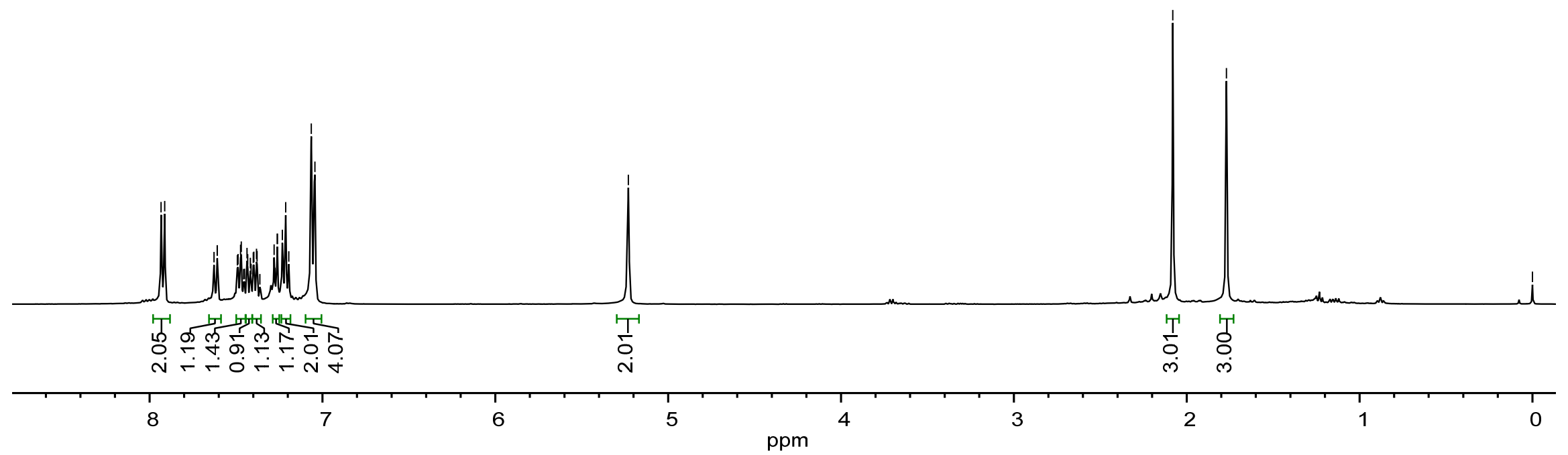




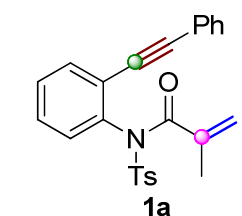

${ }^{13} \mathrm{C} \mathrm{NMR}\left(\mathrm{CDCl}_{3}, 100 \mathrm{MHz}\right)$

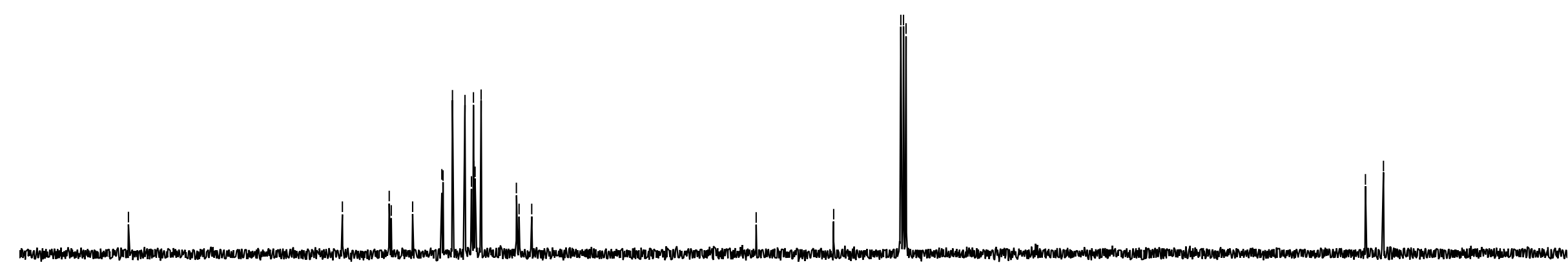

\begin{tabular}{|c|c|c|c|c|c|c|c|c|c|}
\hline 1 & 1 & 1 & 1 & 1 & $T$ & $T$ & $T$ & $T$ & $T$ \\
\hline 180 & 160 & 140 & 120 & 100 & 80 & 60 & 40 & 20 & 0 \\
\hline
\end{tabular}



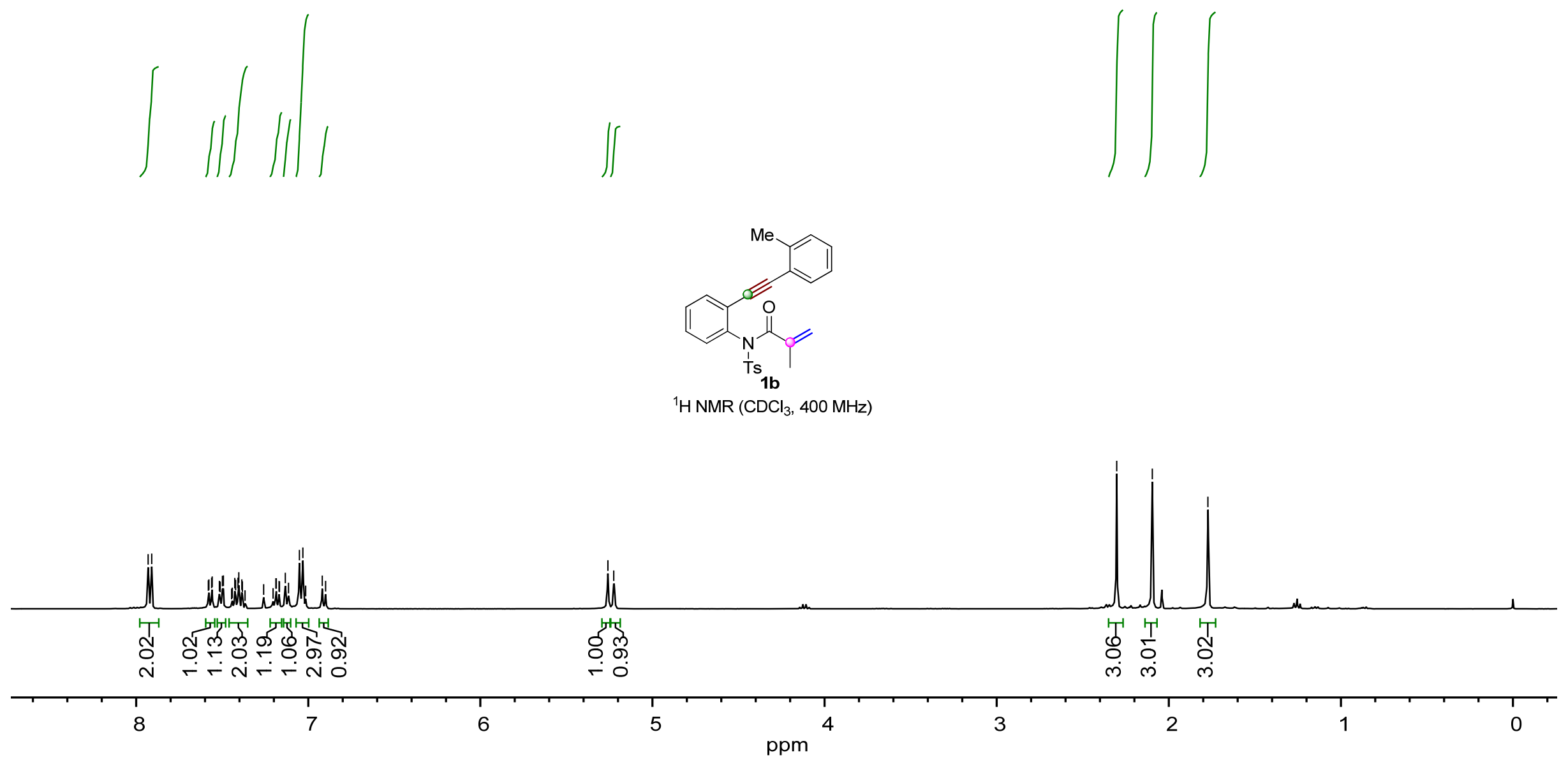


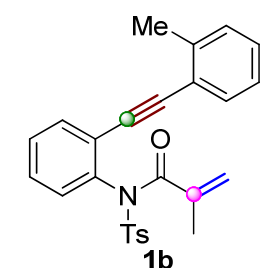

$\left.{ }^{13} \mathrm{C} \mathrm{NMR} \mathrm{(CDCl}, 100 \mathrm{MHz}\right)$

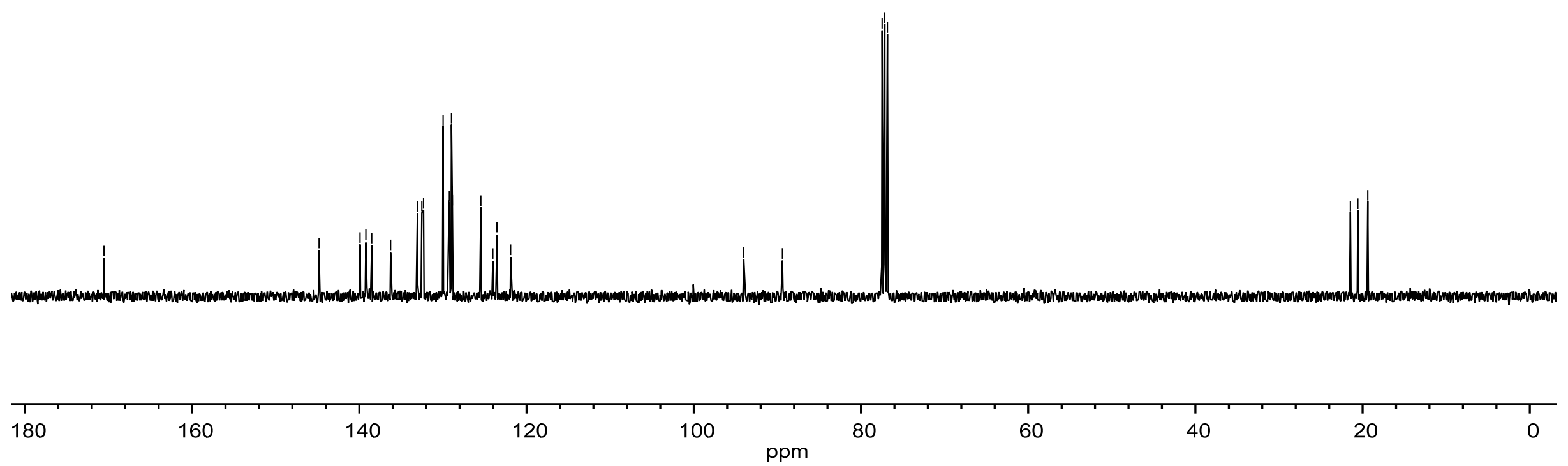



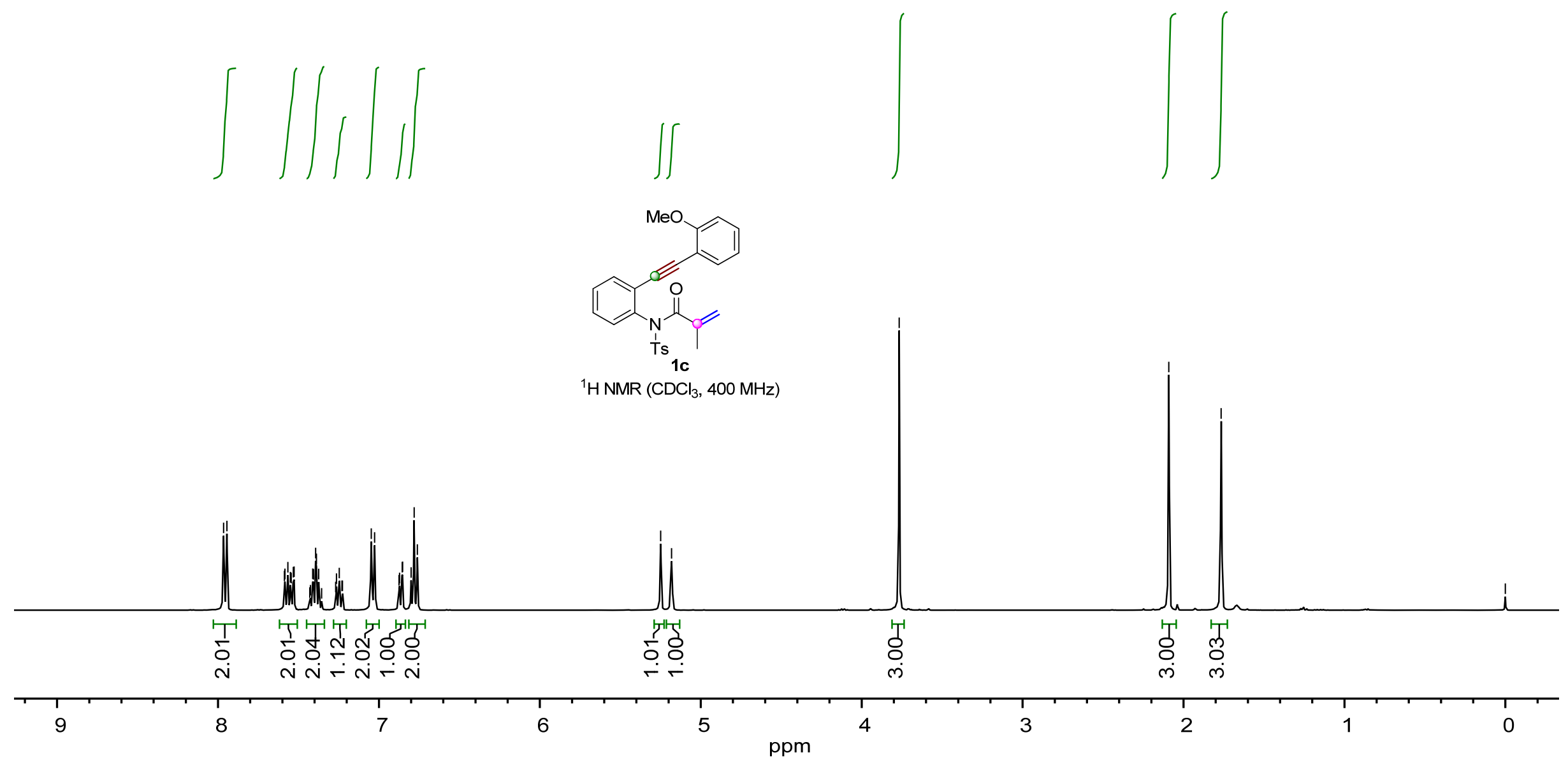


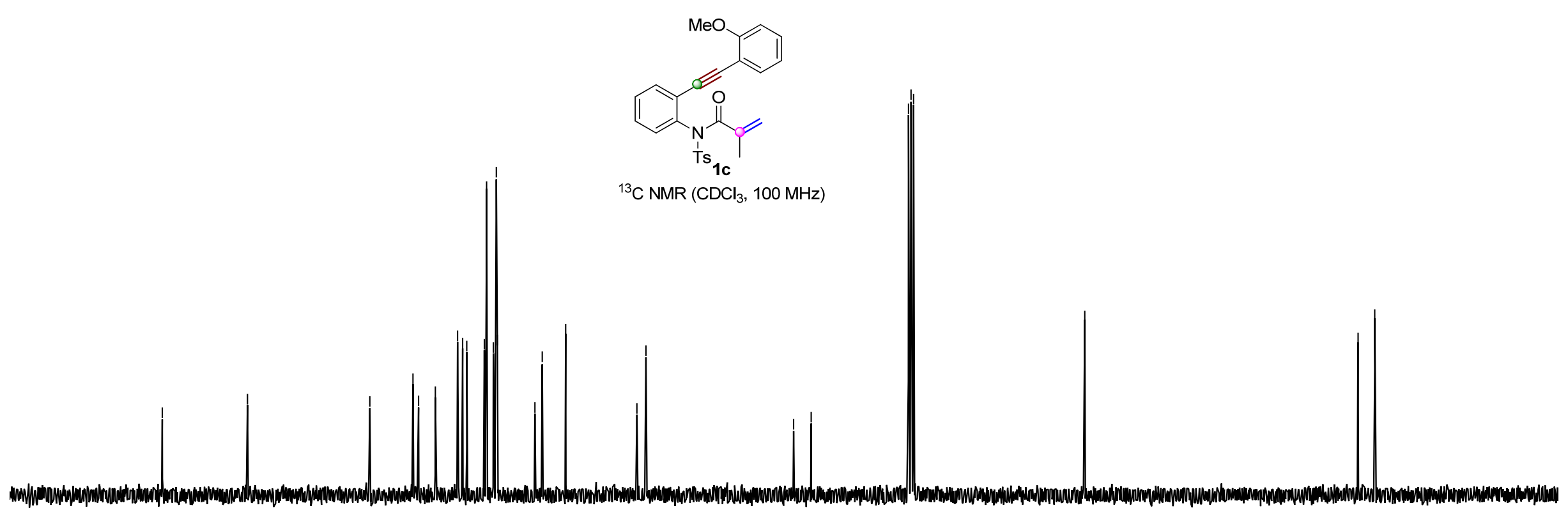

\begin{tabular}{|c|c|c|c|c|c|c|c|c|}
\hline 180 & 160 & 140 & 120 & 100 & 80 & 60 & 40 & 20 \\
\hline
\end{tabular}



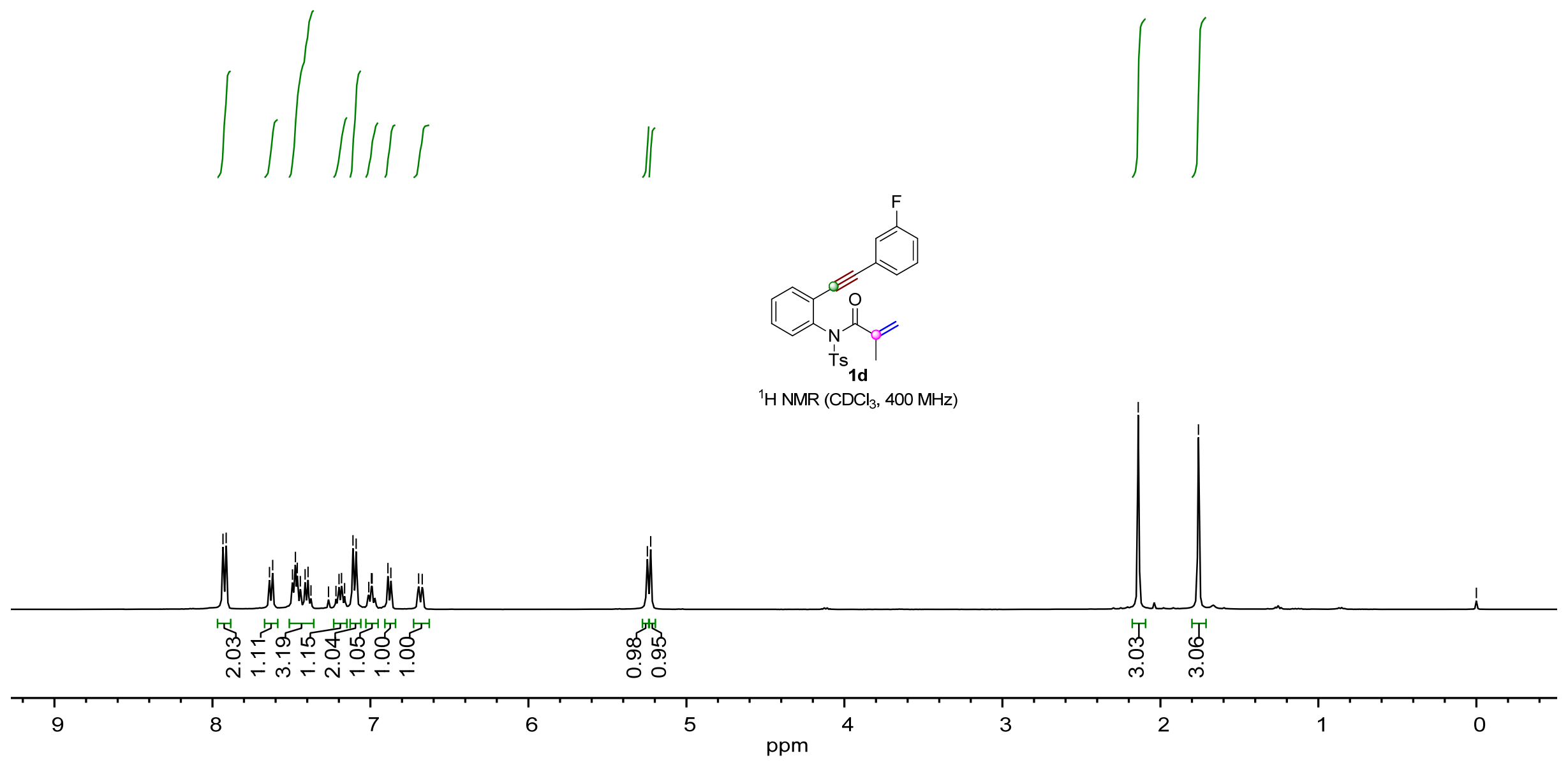
:

定

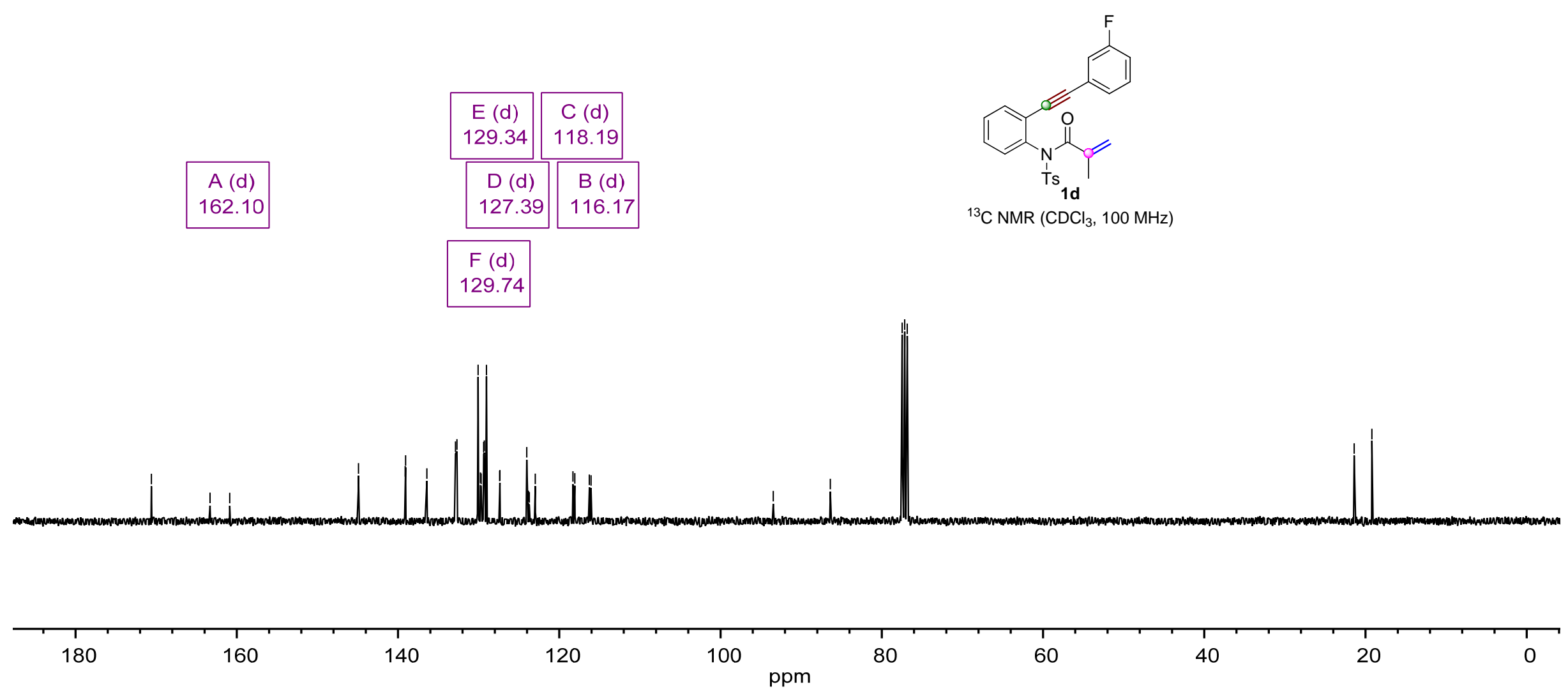


$\infty$
$\stackrel{\infty}{I}$
$\stackrel{7}{7}$

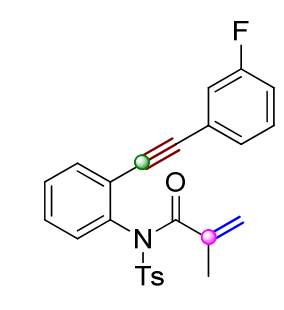

${ }^{19} \mathrm{~F} \mathrm{NMR}\left(\mathrm{CDCl}_{3}, 376 \mathrm{MHz}\right)$

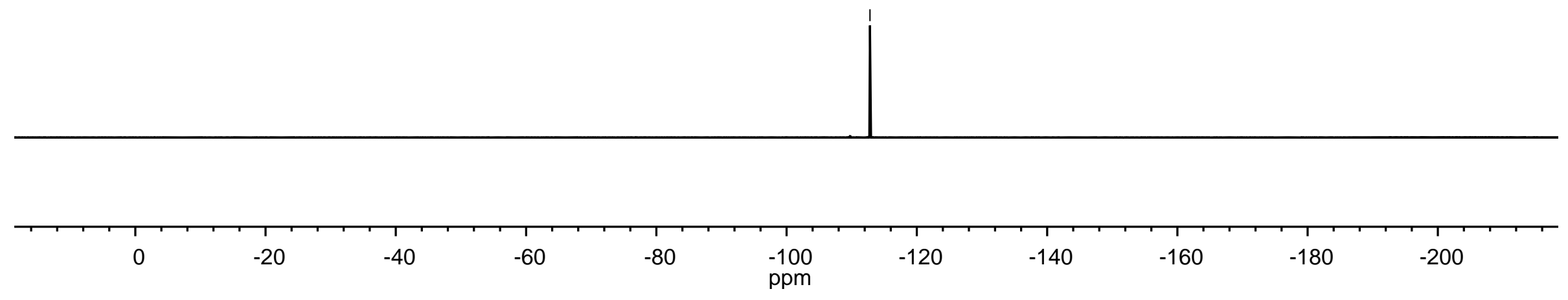




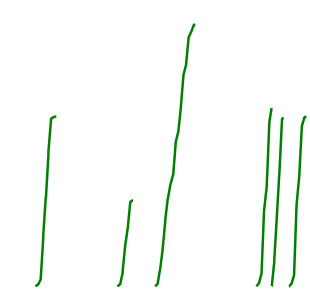

$\|$
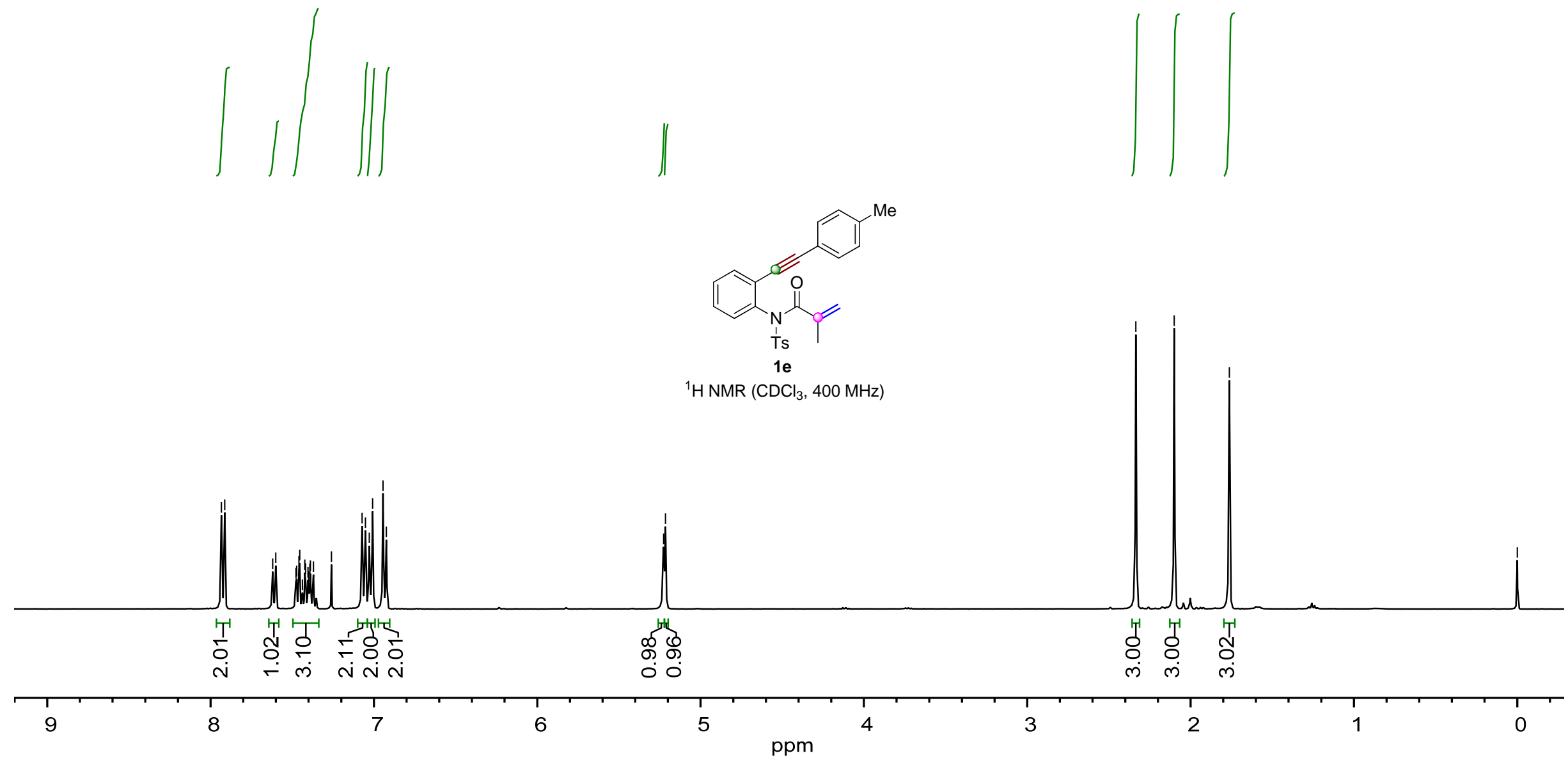


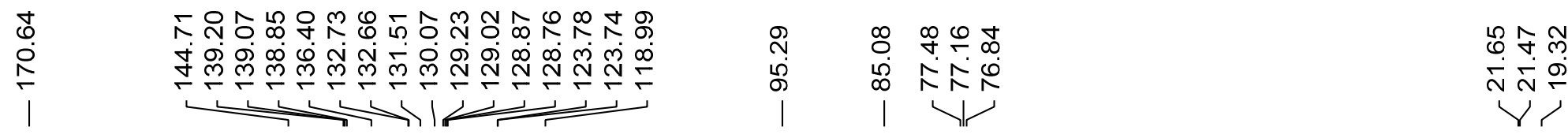

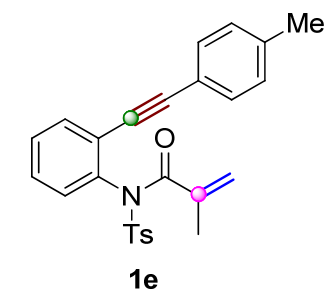

${ }^{13} \mathrm{C} \mathrm{NMR}\left(\mathrm{CDCl}_{3}, 100 \mathrm{MHz}\right)$

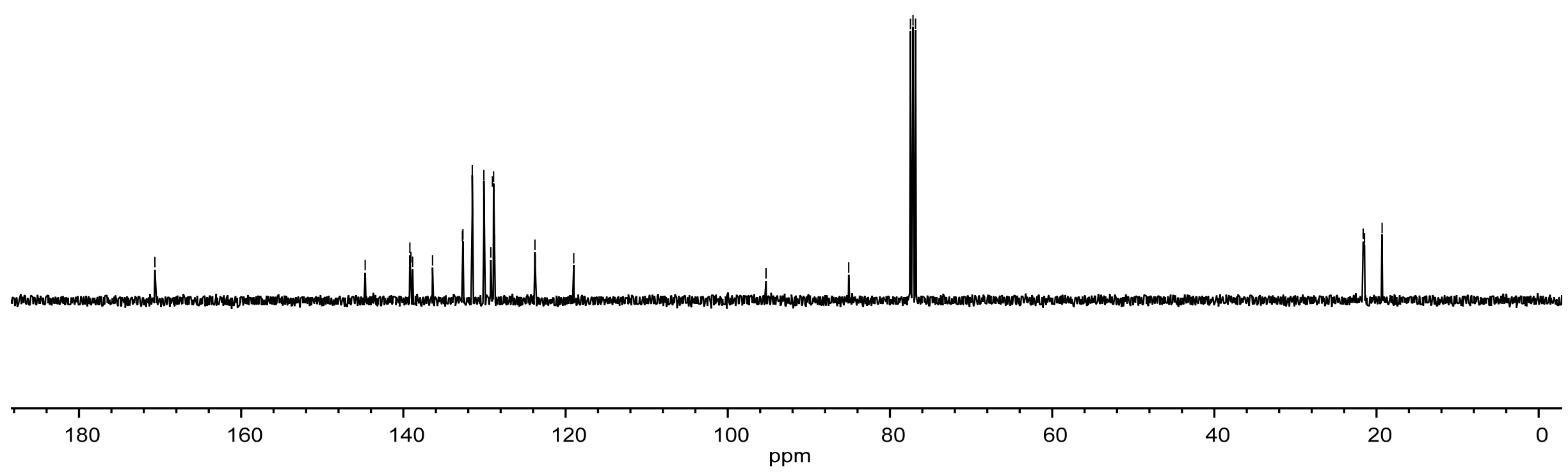




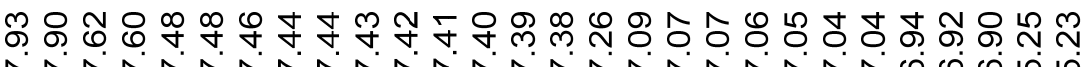

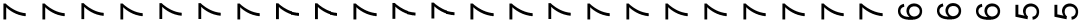
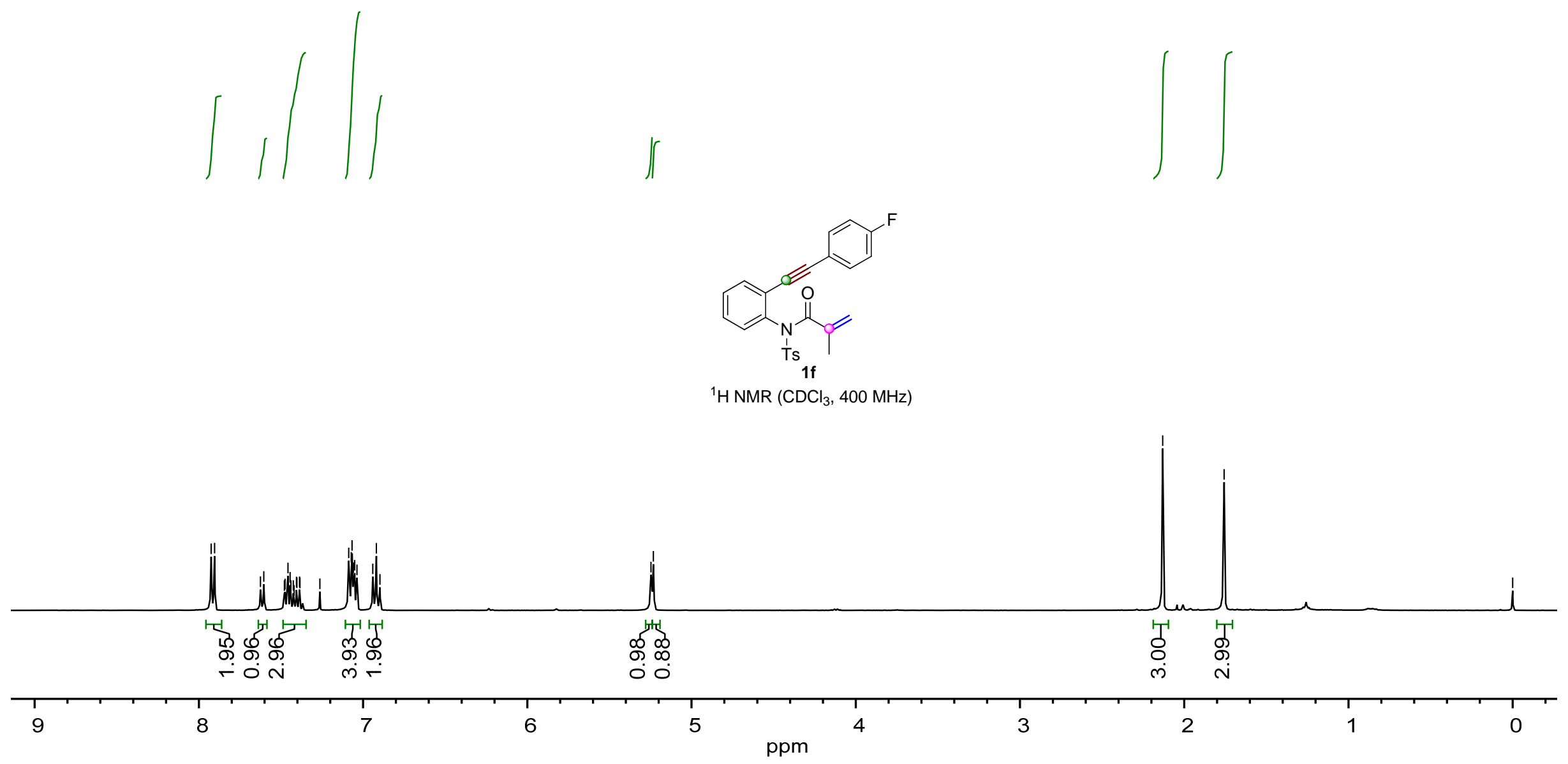


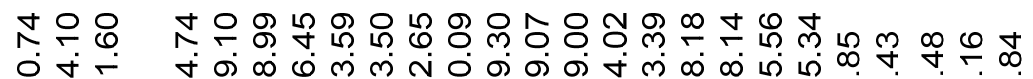

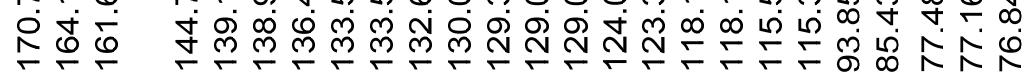

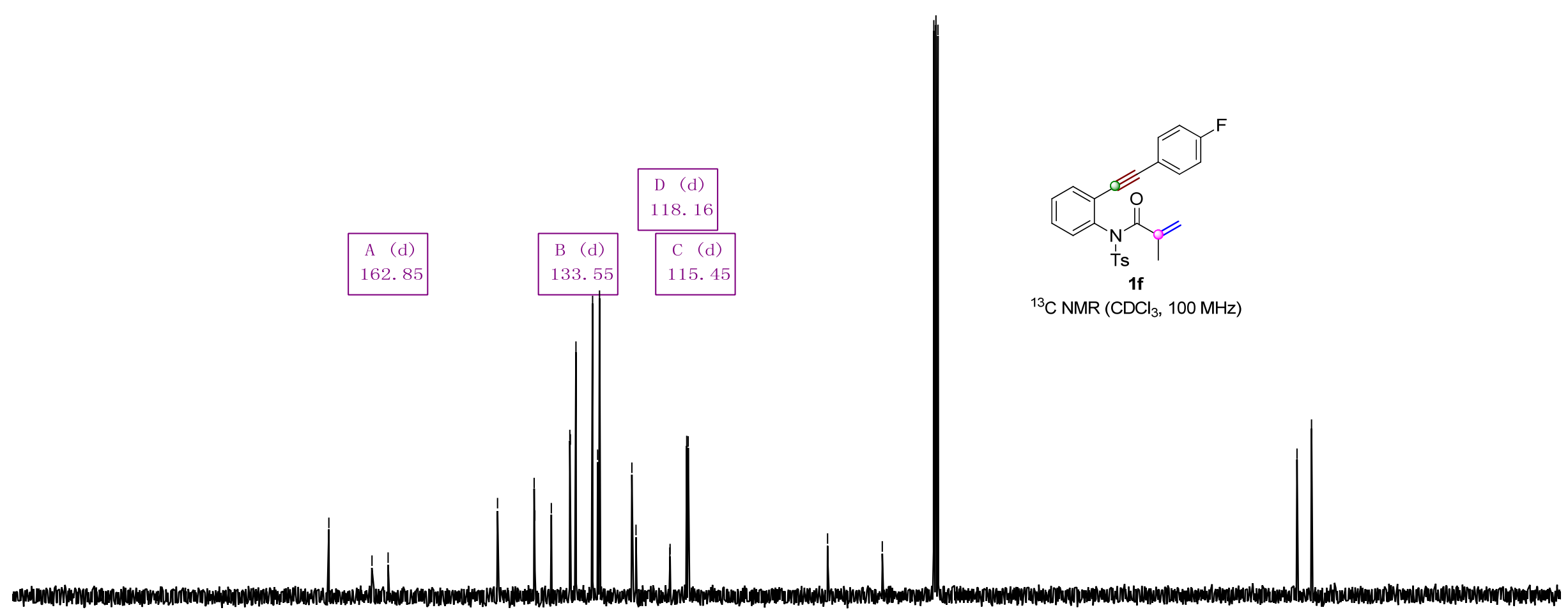

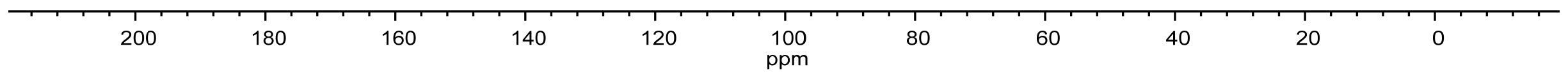



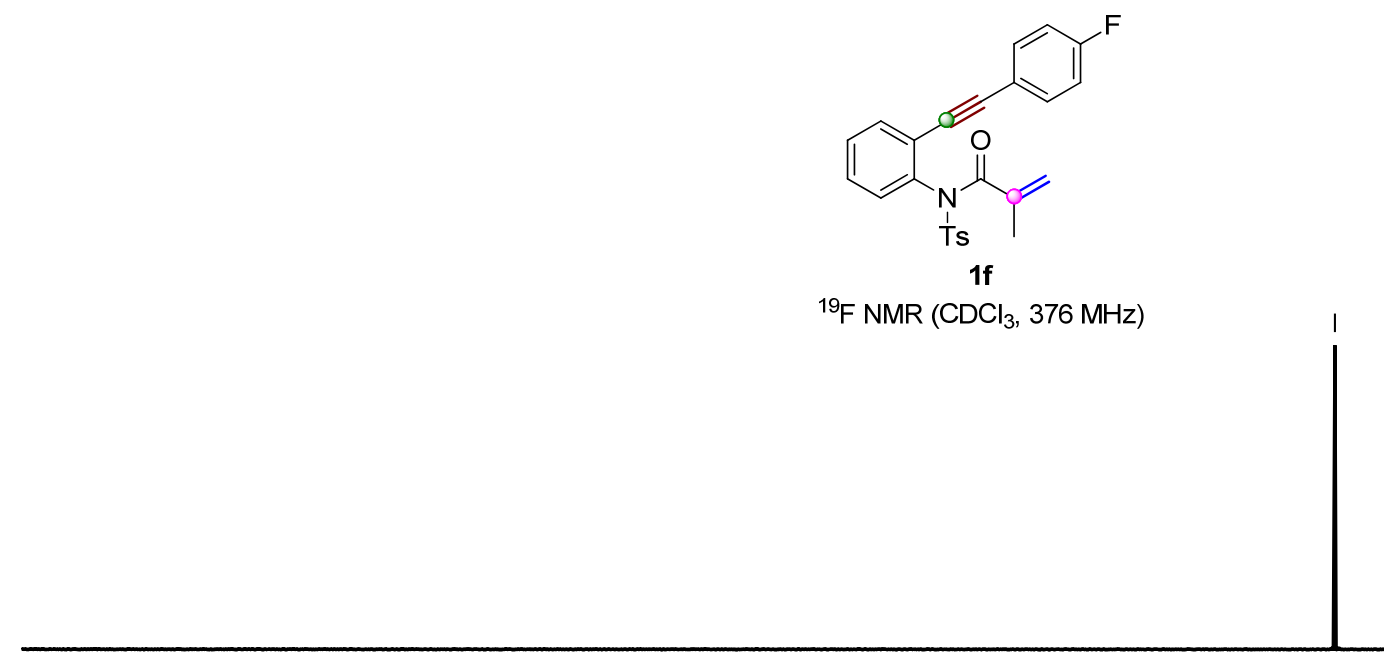

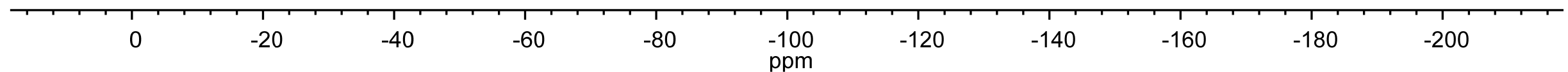




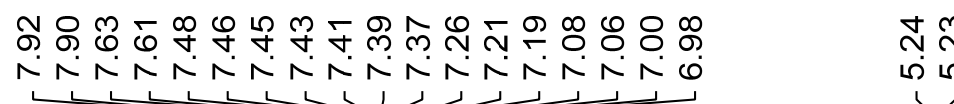

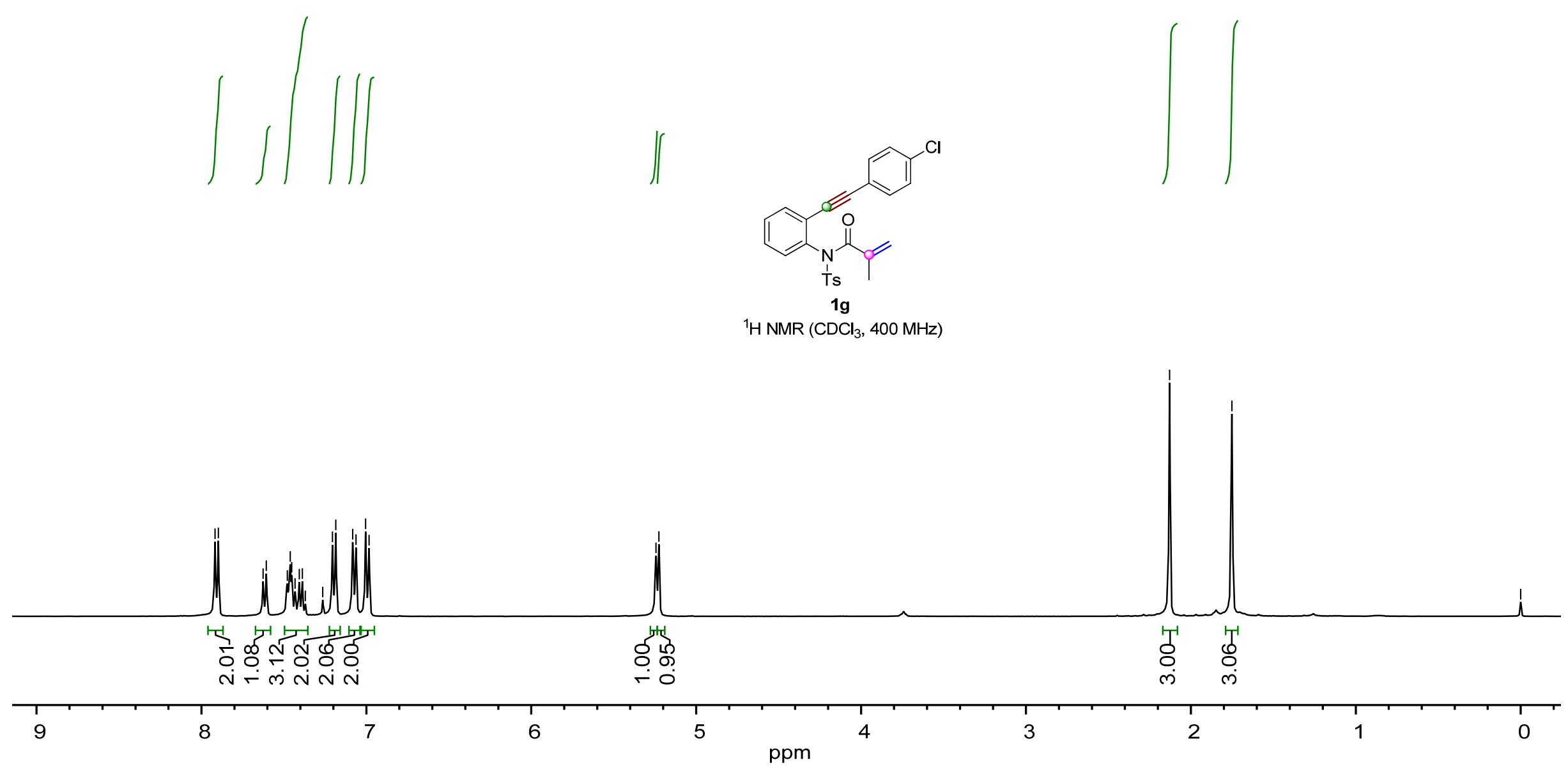

$\stackrel{m}{\stackrel{n}{r}} \stackrel{\frac{n}{r}}{1}$ 


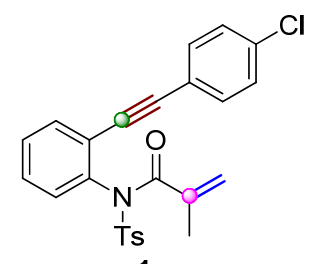

$1 \mathrm{~g}$

${ }^{13} \mathrm{C} \mathrm{NMR}\left(\mathrm{CDCl}_{3}, 100 \mathrm{MHz}\right)$

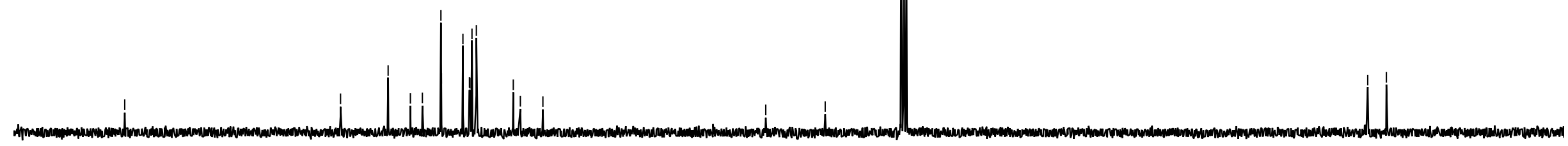




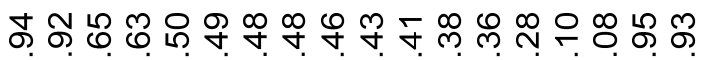

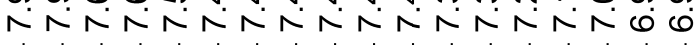
$\longrightarrow$

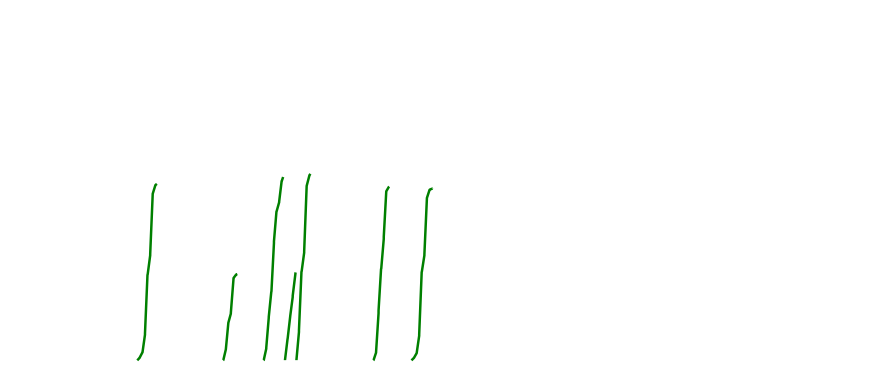

กิ

(1)

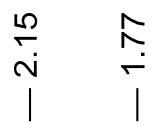

ํ.

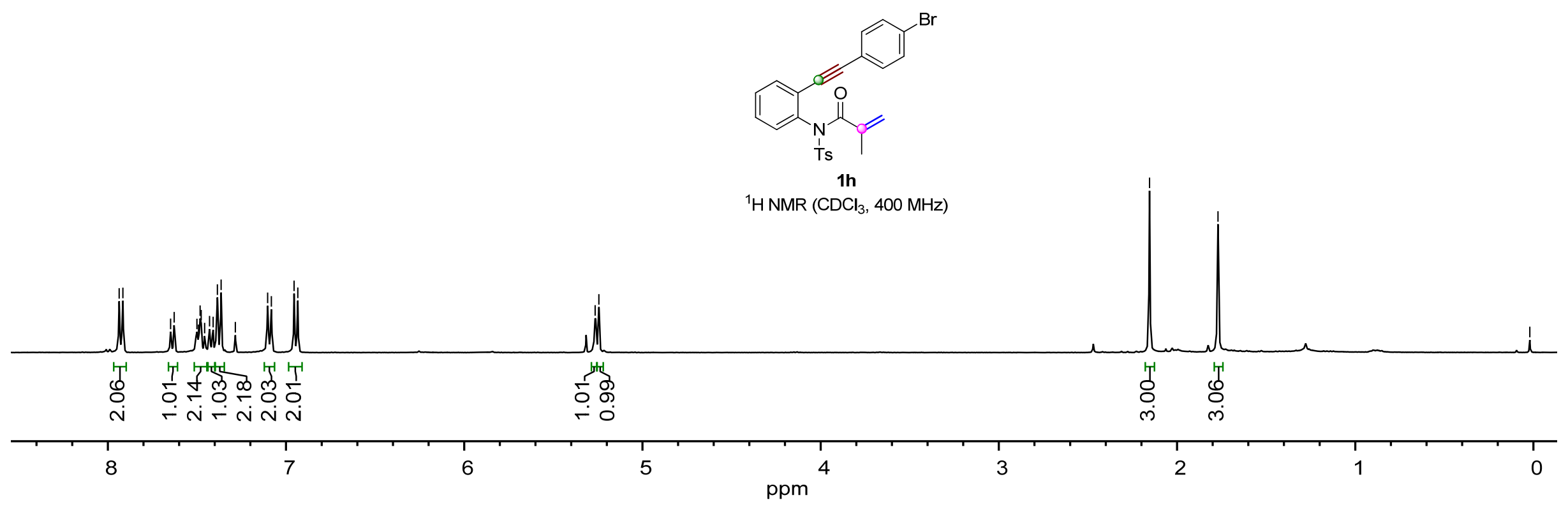




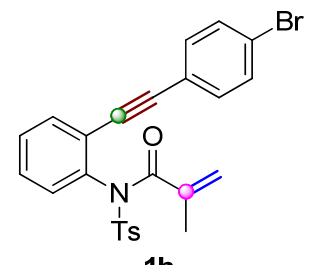

${ }^{13} \mathrm{C} \mathrm{NMR}\left(\mathrm{CDCl}_{3}, 100 \mathrm{MHz}\right)$

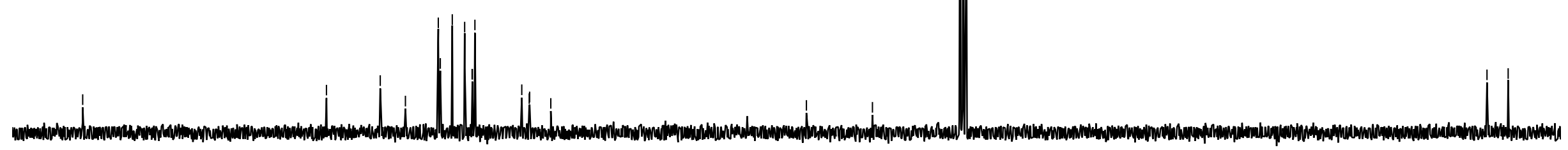

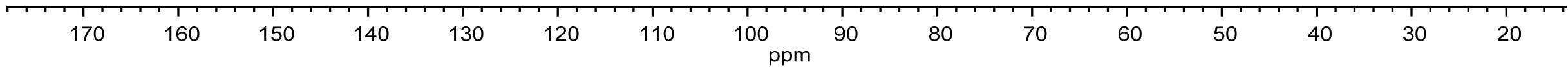


穴

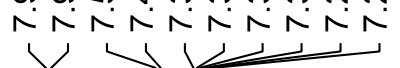

กู่

iก

$\int 1$

J

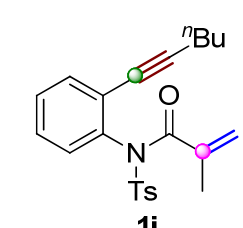

${ }^{1} \mathrm{H} \mathrm{NMR}\left(\mathrm{CDCl}_{3}, 400 \mathrm{MHz}\right)$

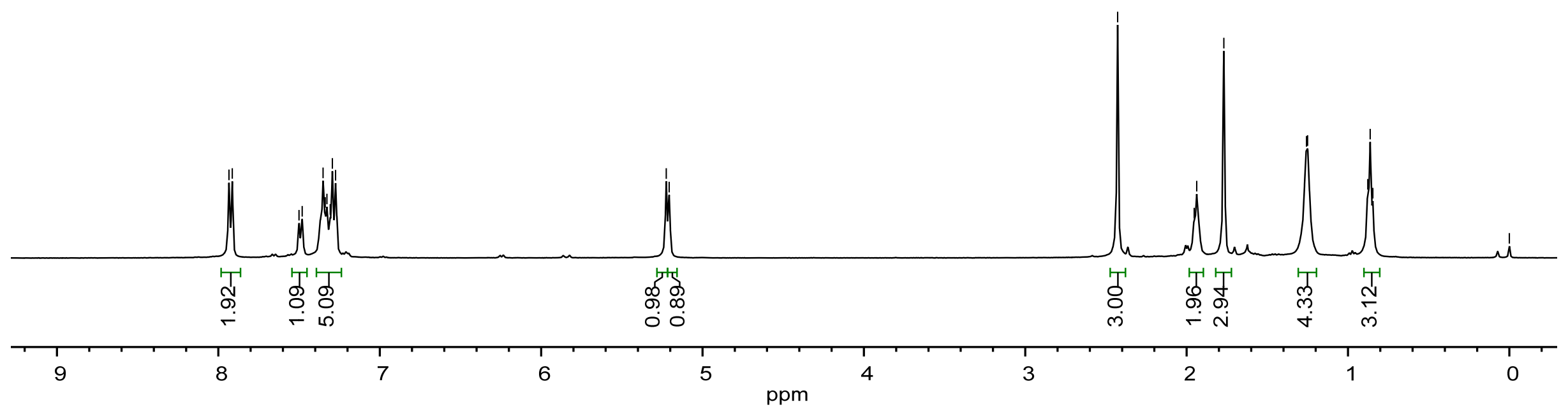

لَ

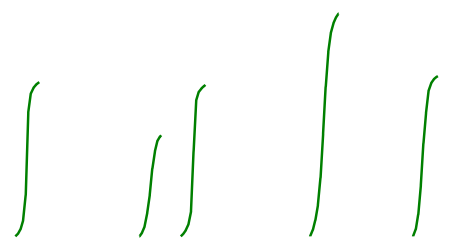




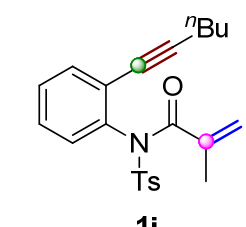

${ }^{13} \mathrm{C} \mathrm{NMR}\left(\mathrm{CDCl}_{3}, 100 \mathrm{MHz}\right)$

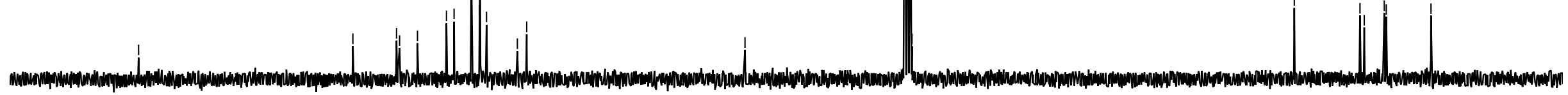

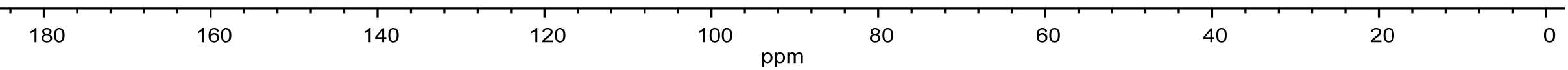




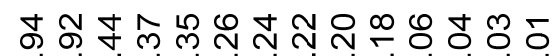

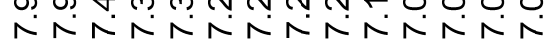

$\checkmark$ 管
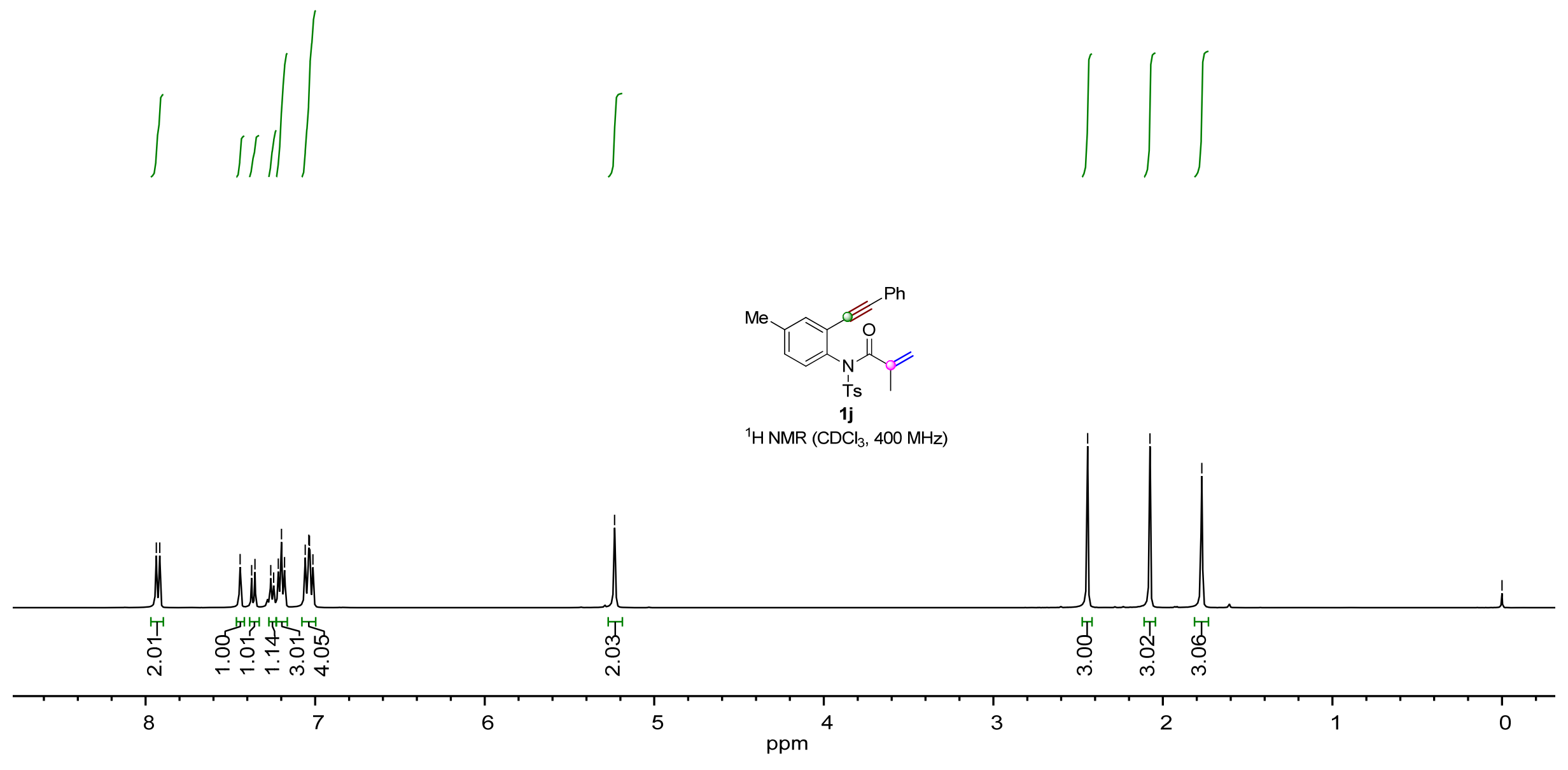

${ }^{1} \mathrm{H} \mathrm{NMR}\left(\mathrm{CDCl}_{3}, 400 \mathrm{MHz}\right)$

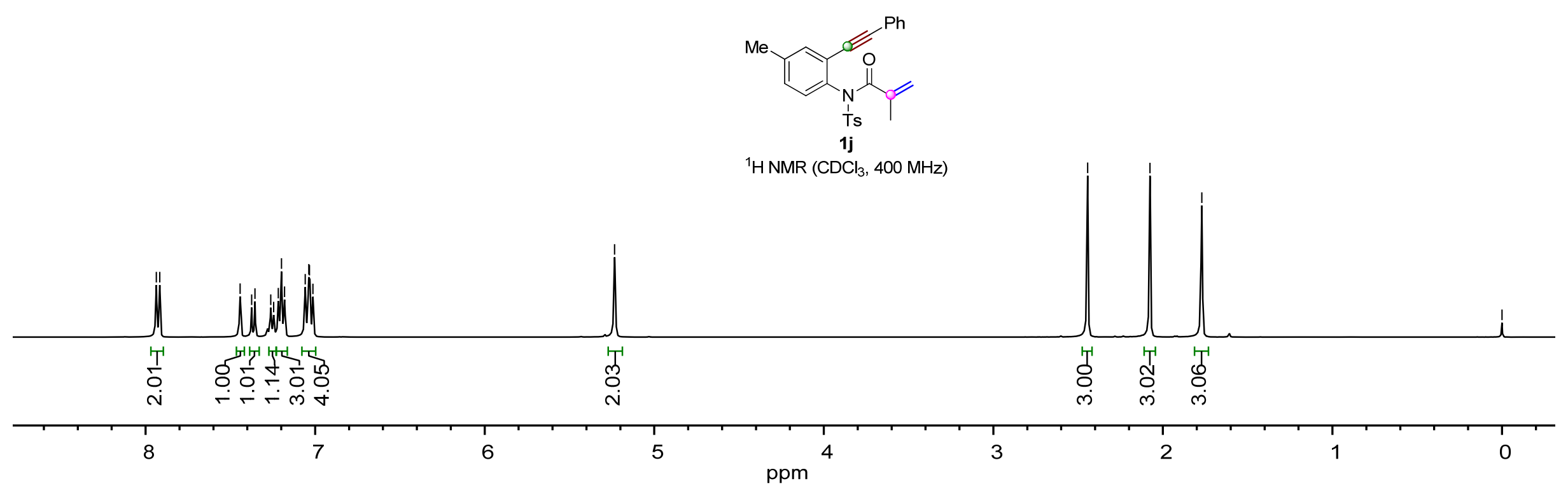

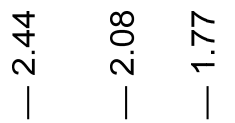

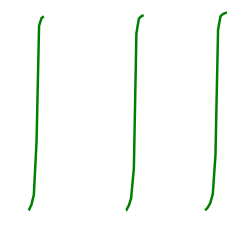




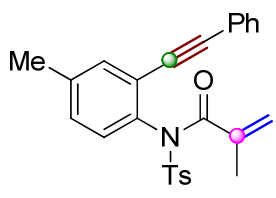

1j

${ }^{13} \mathrm{C} \mathrm{NMR}\left(\mathrm{CDCl}_{3}, 100 \mathrm{MHz}\right)$
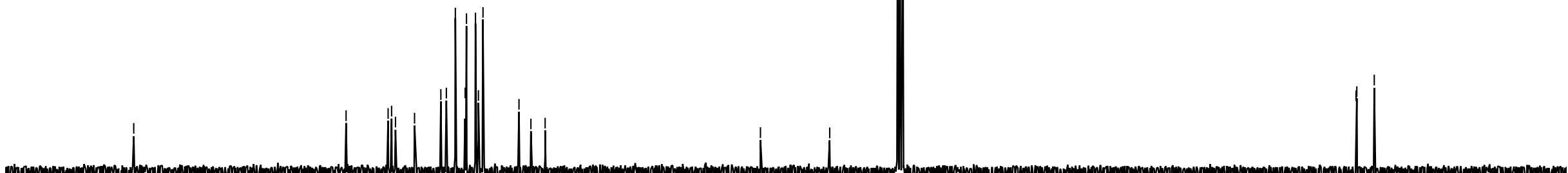

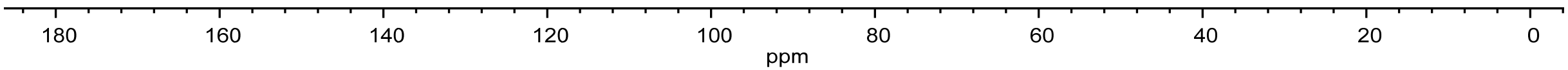


๙ু

ำ

เก

ㅇำ

i
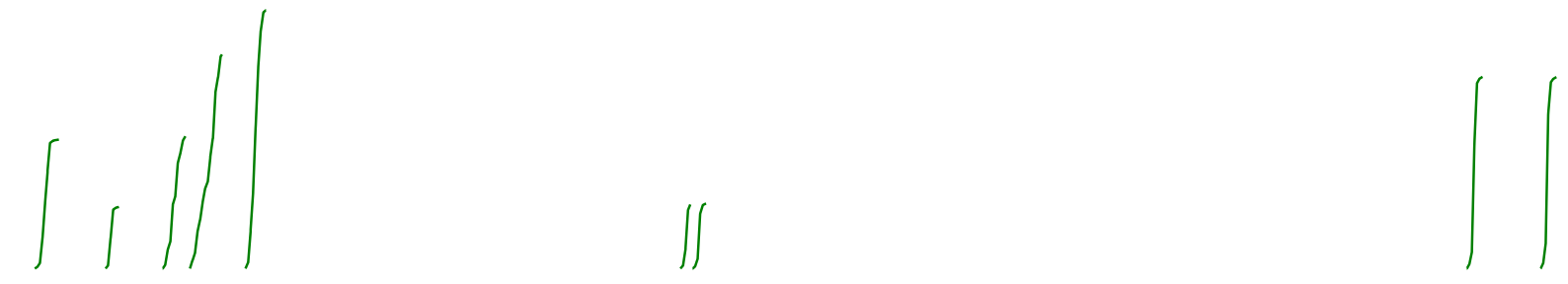

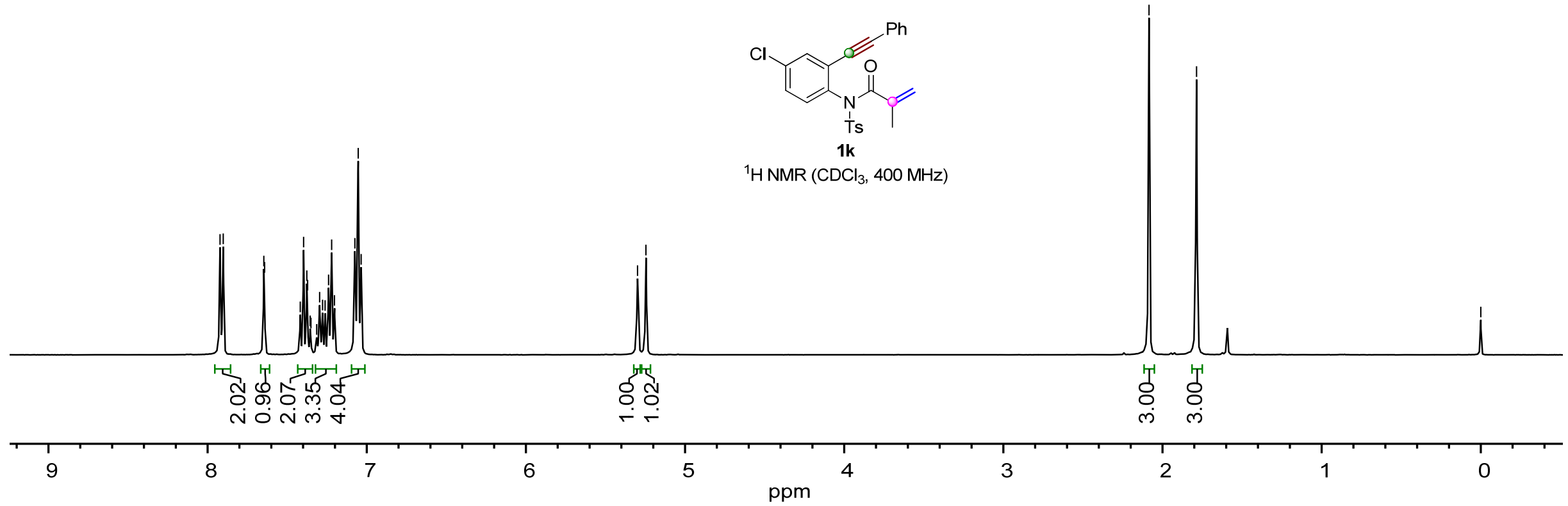




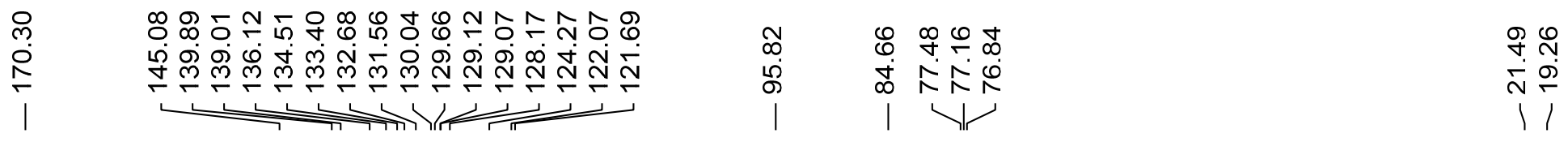

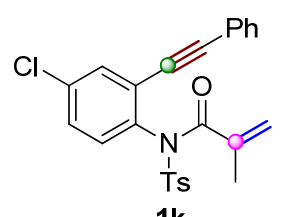

${ }^{13} \mathrm{C} \mathrm{NMR}\left(\mathrm{CDCl}_{3}, 100 \mathrm{MHz}\right)$

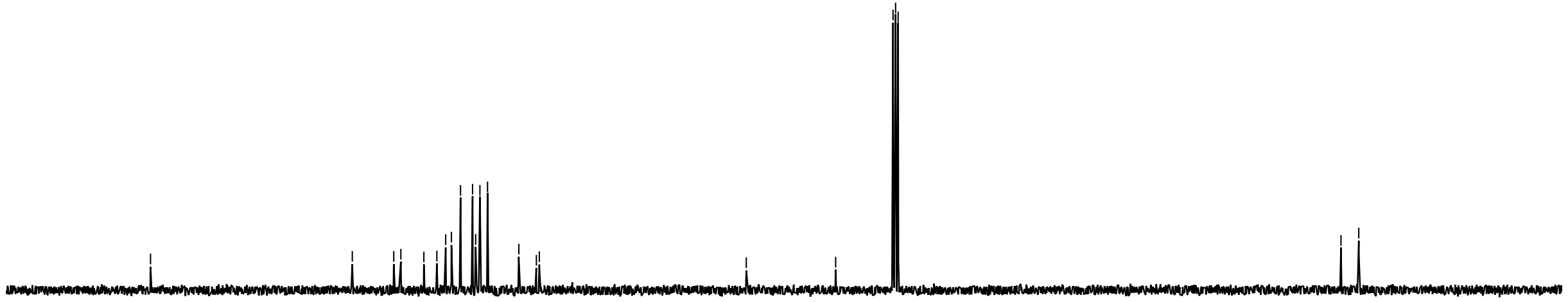

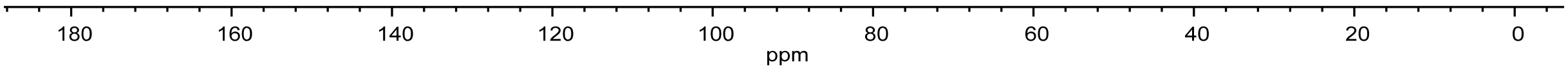



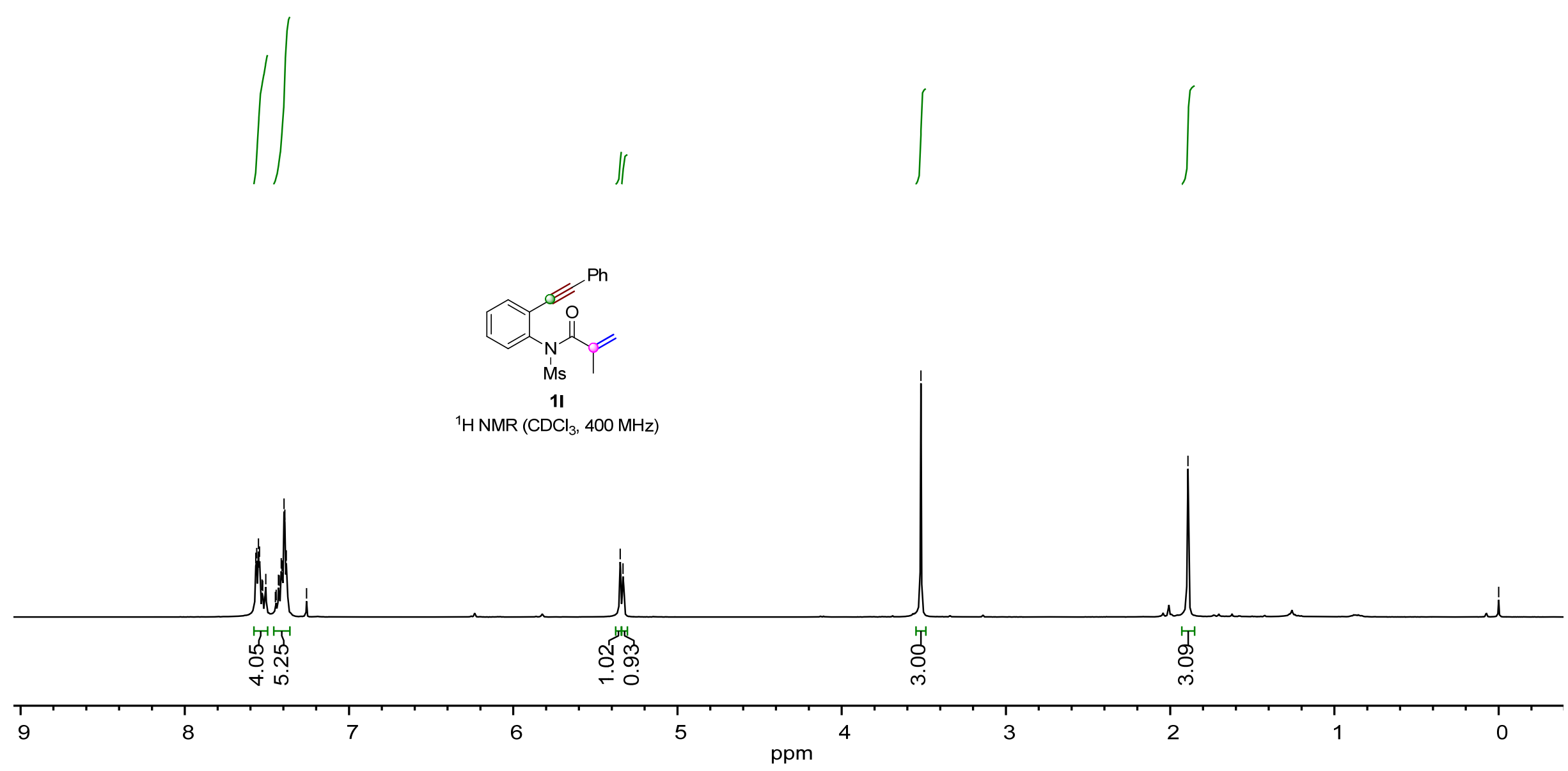


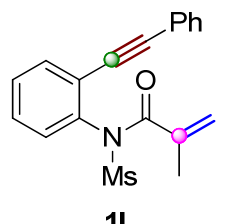

${ }^{13} \mathrm{C} \mathrm{NMR}\left(\mathrm{CDCl}_{3}, 100 \mathrm{MHz}\right)$

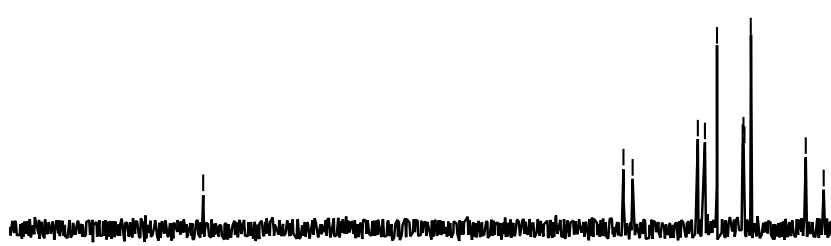
C.

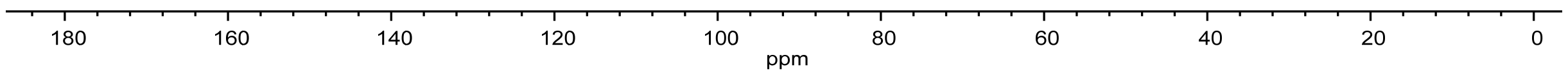



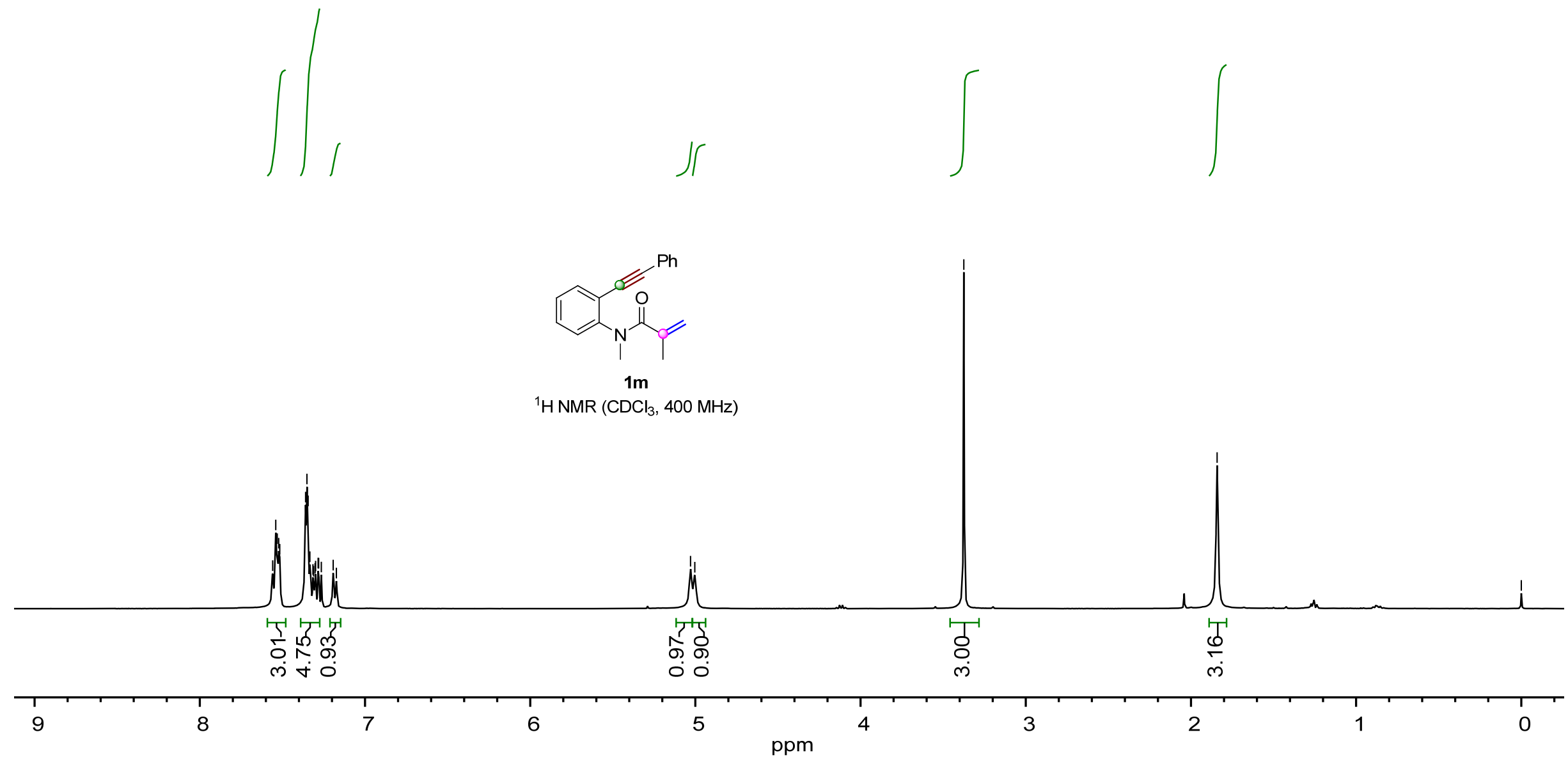


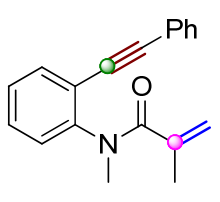

$1 \mathrm{~m}$

${ }^{13} \mathrm{C} \mathrm{NMR}\left(\mathrm{CDCl}_{3}, 100 \mathrm{MHz}\right)$
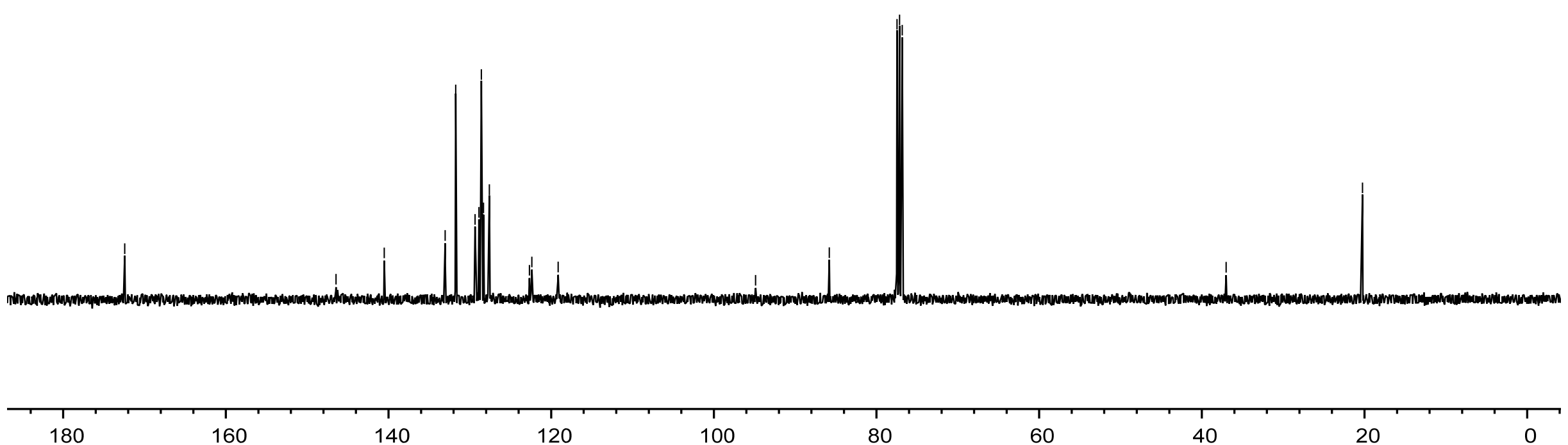
ำ

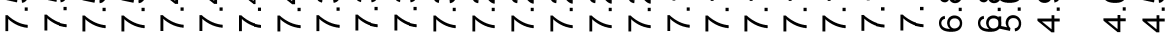

I) I

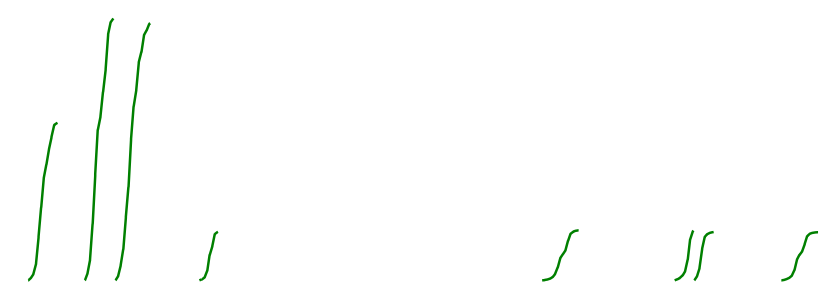

$\int$

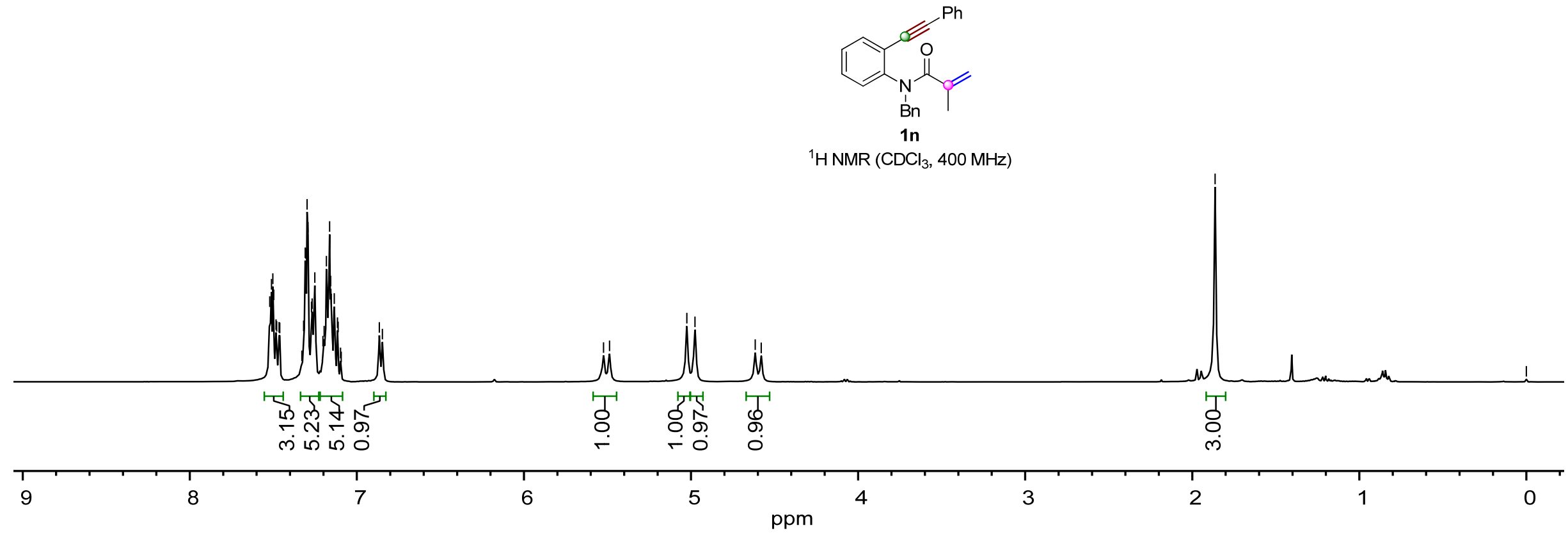



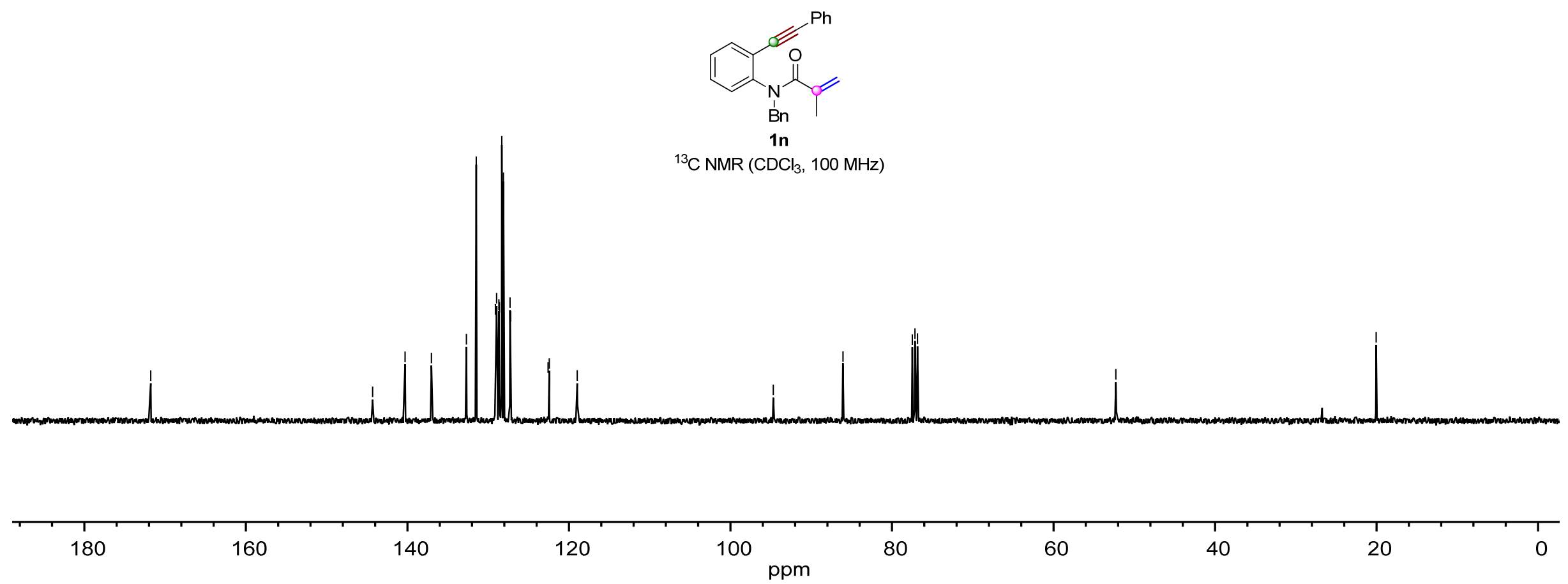
ชิ

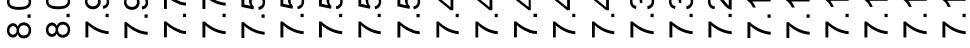

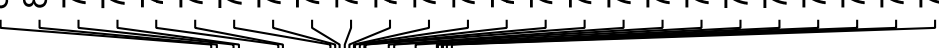

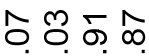

نं $\dot{\nabla}$

11

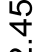

(

$\infty$
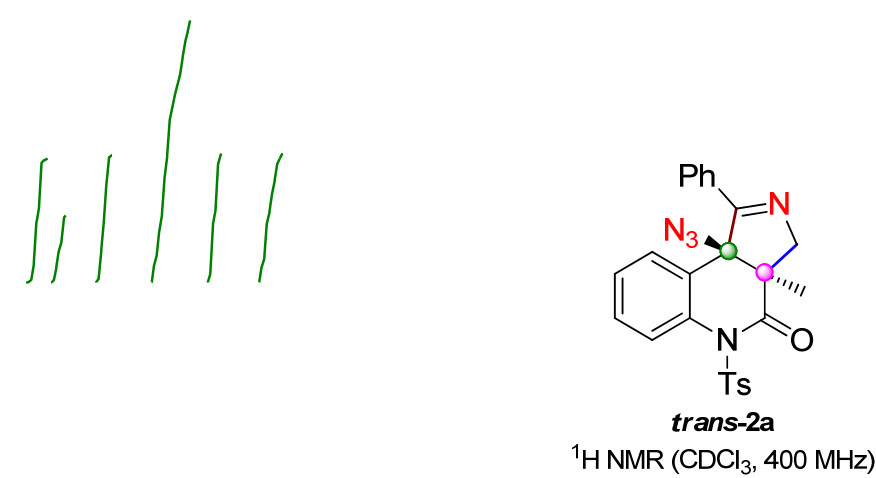

$\int\{$

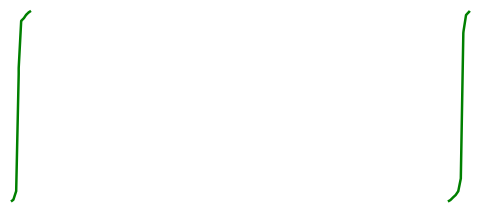

${ }^{1} \mathrm{H} \mathrm{NMR}\left(\mathrm{CDCl}_{3}, 400 \mathrm{MHz}\right)$

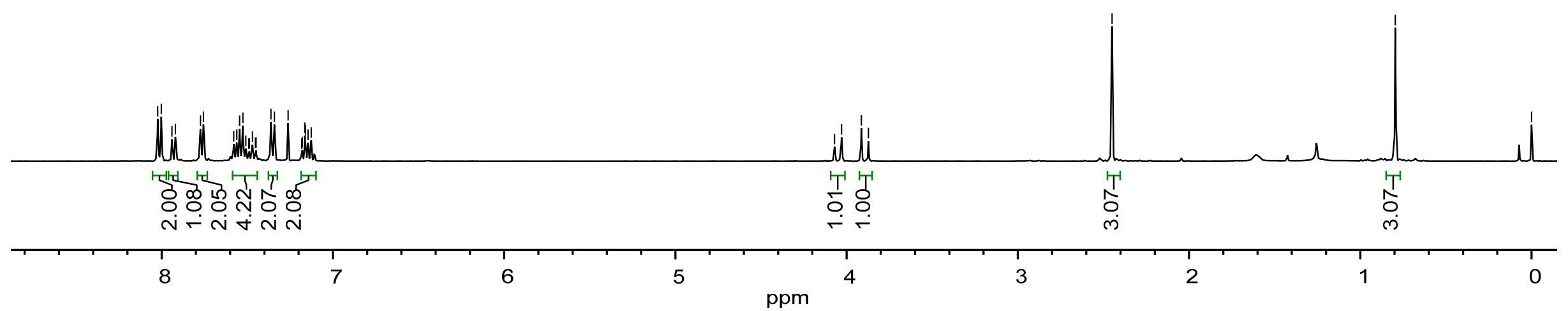




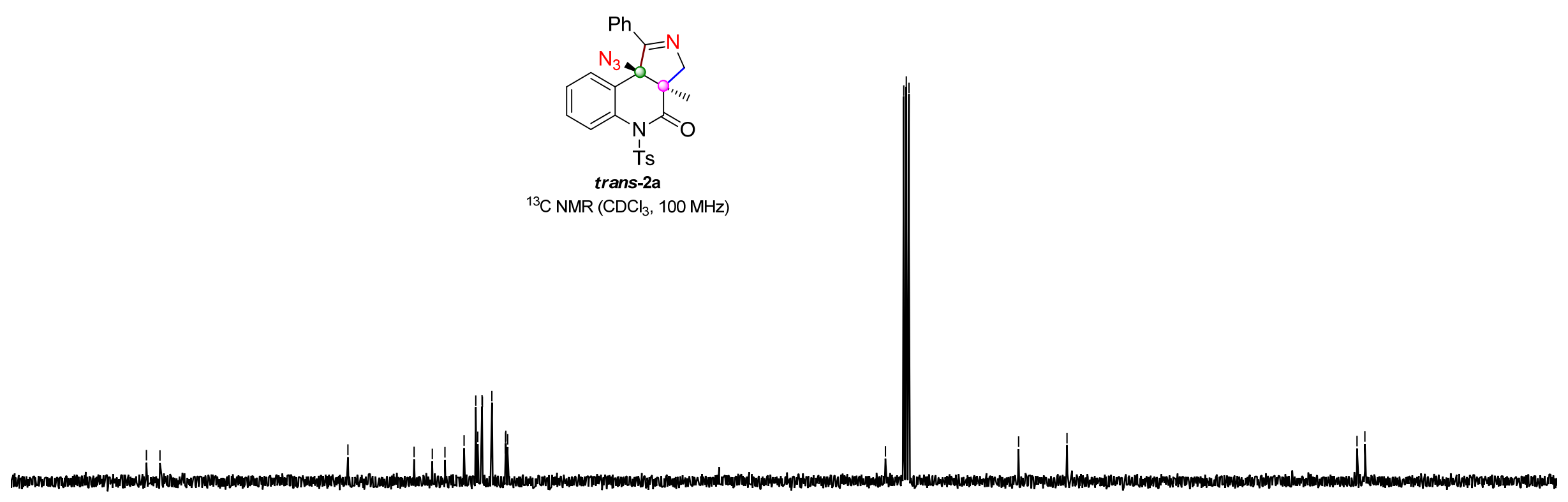




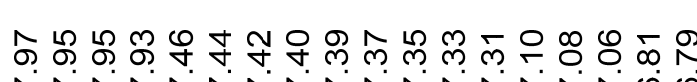

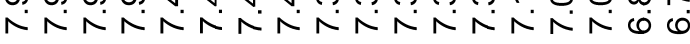

4 (1)
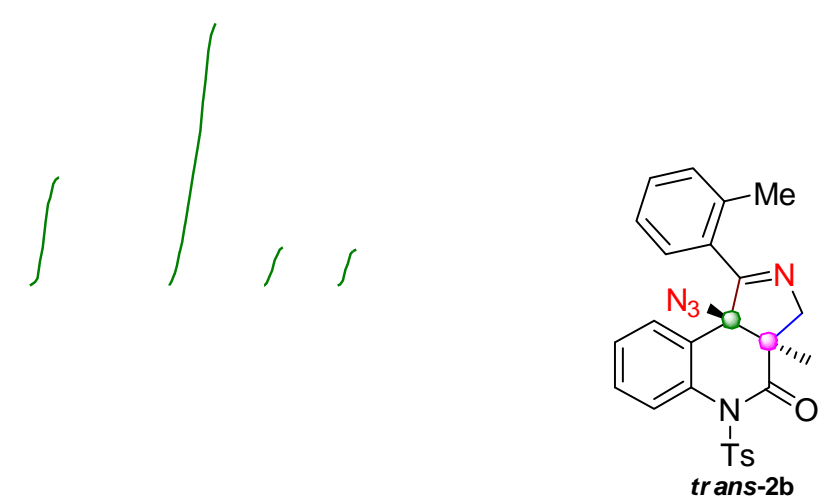

${ }^{1} \mathrm{H} \mathrm{NMR}\left(\mathrm{CDCl}_{3}, 400 \mathrm{MHz}\right)$

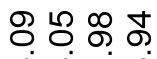

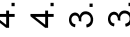

i)

䇶

ก่

๖ั

।

8

$\stackrel{8}{\circ}$

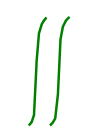

$\int$

de

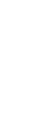

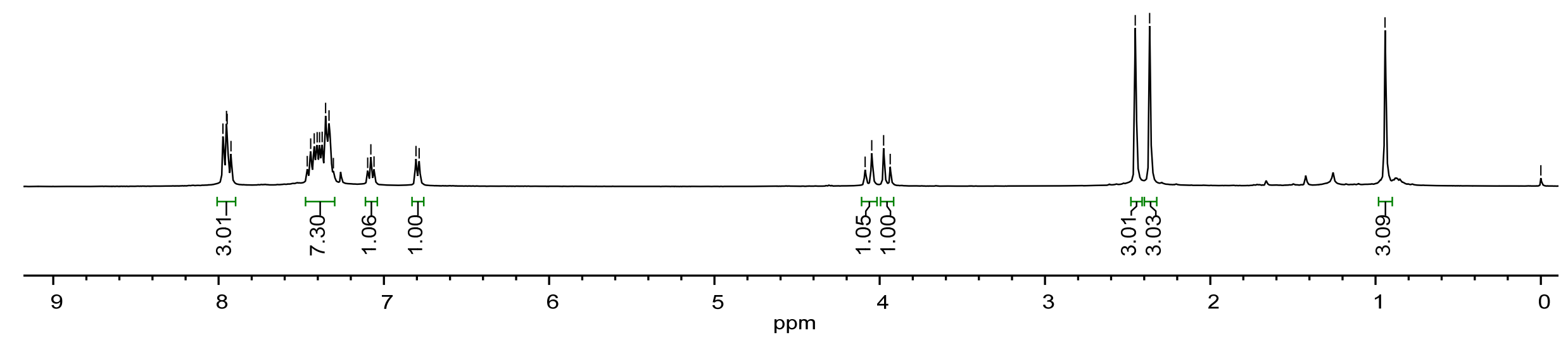

S40 
조

ㅇํㅇ

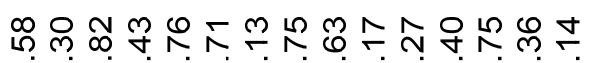

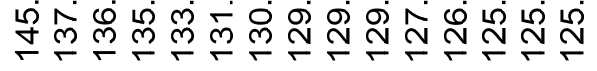

l

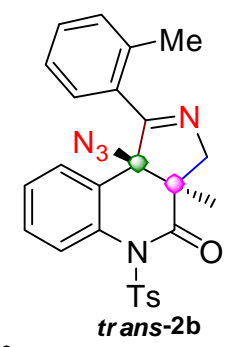

${ }^{3} \mathrm{C}$ NMR $\left(\mathrm{CDCl}_{3}, 100 \mathrm{MHz}\right)$
구요요

䟻是

YI $\infty$

ลักั

$\backslash 1$

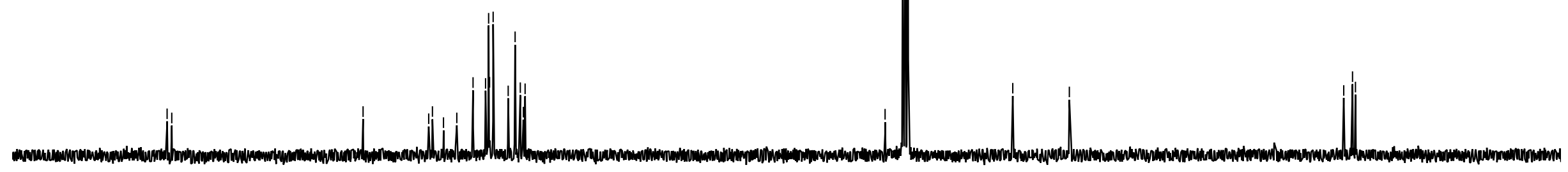

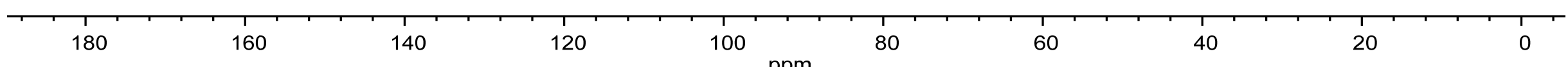




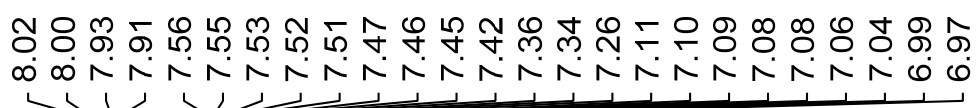

$\mp \infty \infty_{\infty}^{\infty} \infty$

ن
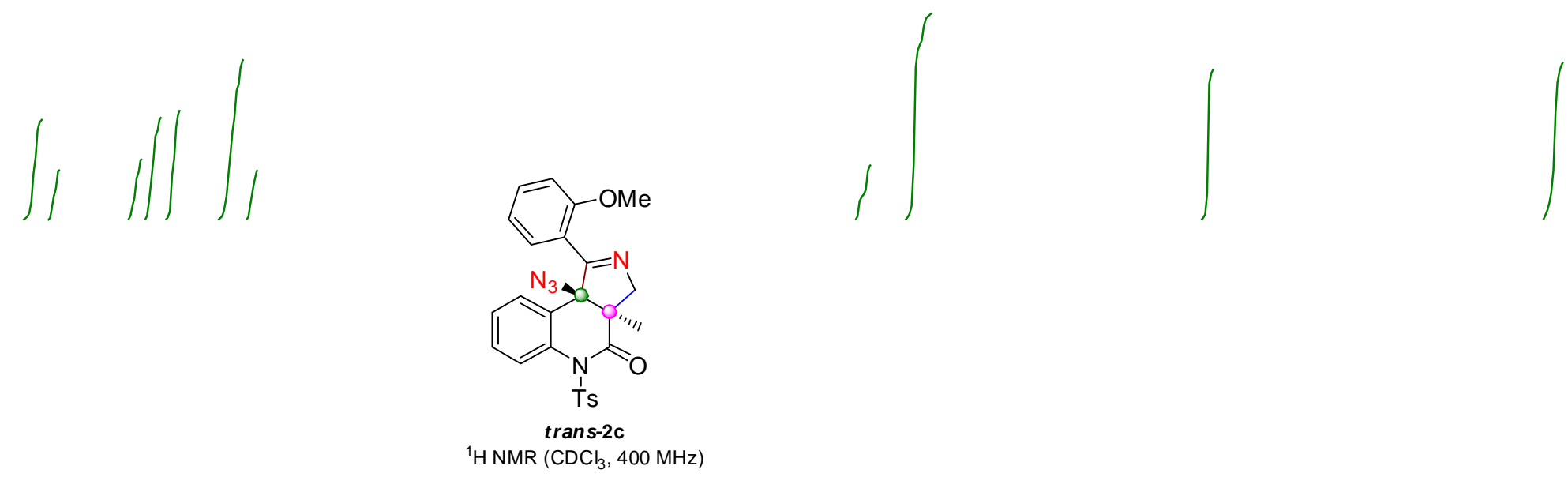

${ }^{1} \mathrm{H}$ NMR $\left(\mathrm{CDCl}_{3}, 400 \mathrm{MHz}\right)$

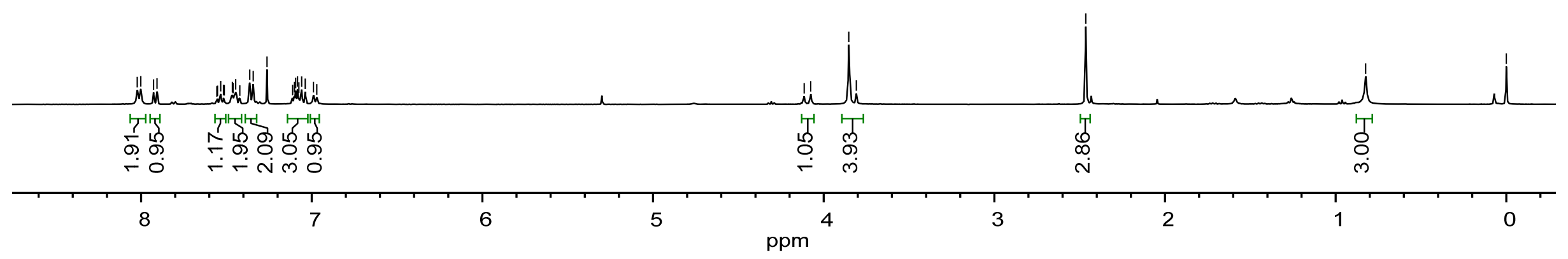




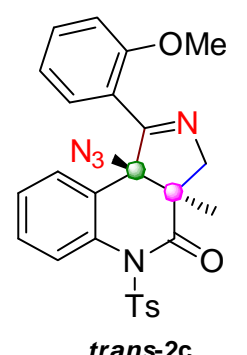

${ }^{13} \mathrm{C}$ NMR $\left(\mathrm{CDCl}_{3}, 100 \mathrm{MHz}\right)$
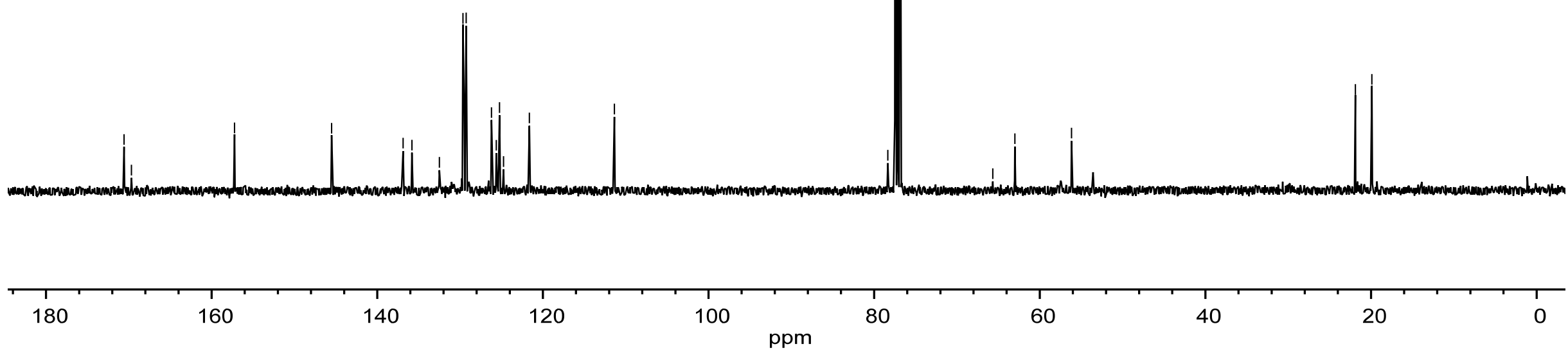


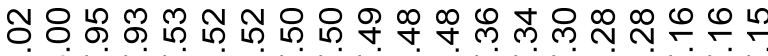

$\infty$

$\Rightarrow$
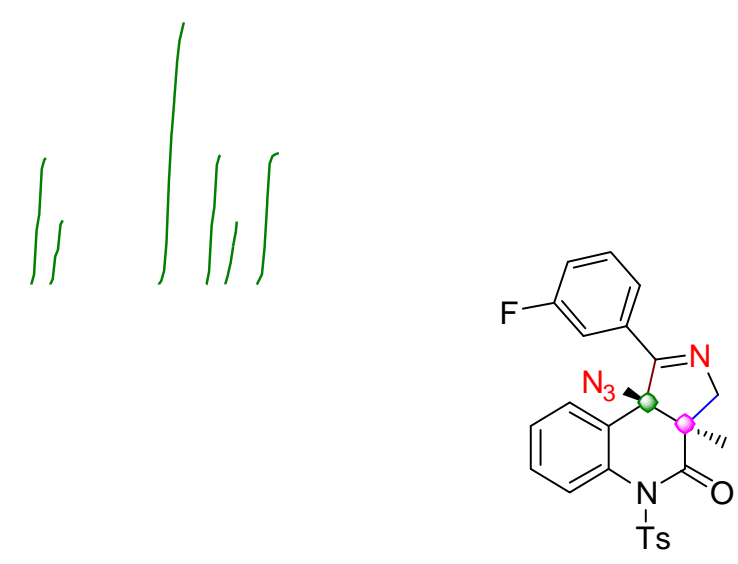

trans-2d

${ }^{1} \mathrm{H} \mathrm{NMR}\left(\mathrm{CDCl}_{3}, 400 \mathrm{MHz}\right)$

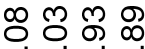

$\dot{\nabla} \dot{\sim} \boldsymbol{m}$

11

$\iint$ $\stackrel{\text { ? }}{\text { N }}$

(

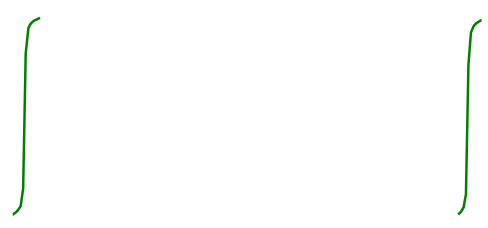

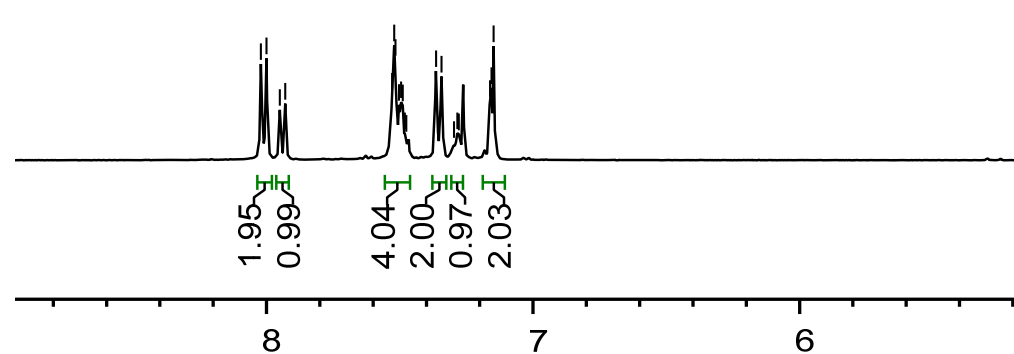

de

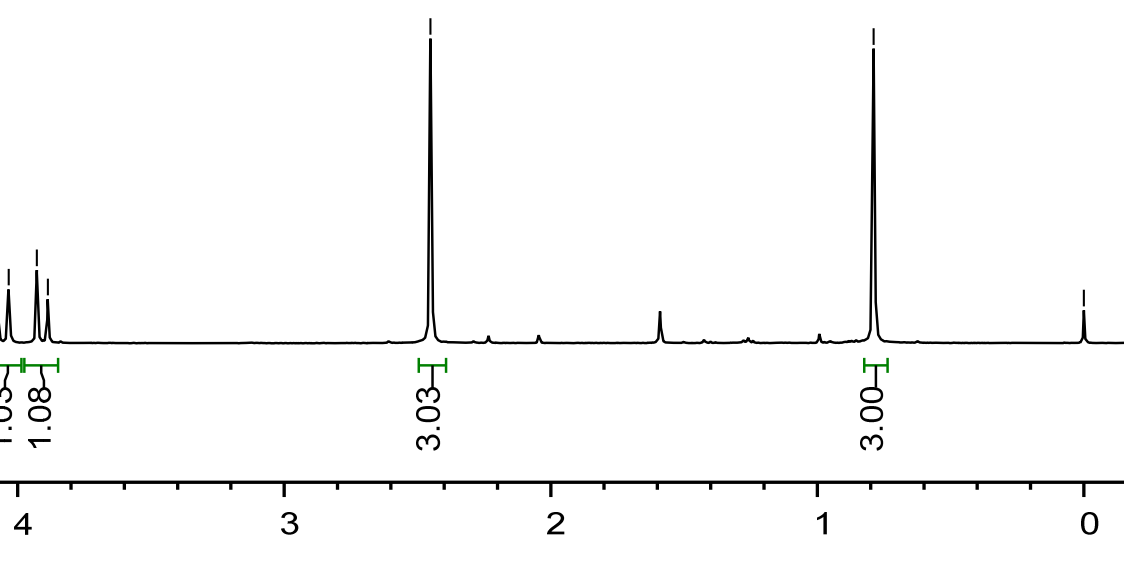


ㄷำ $\stackrel{2}{ก}$

솓워

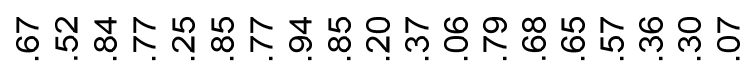

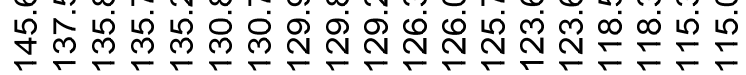

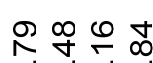

จกิ่

रोI

in

1111

l
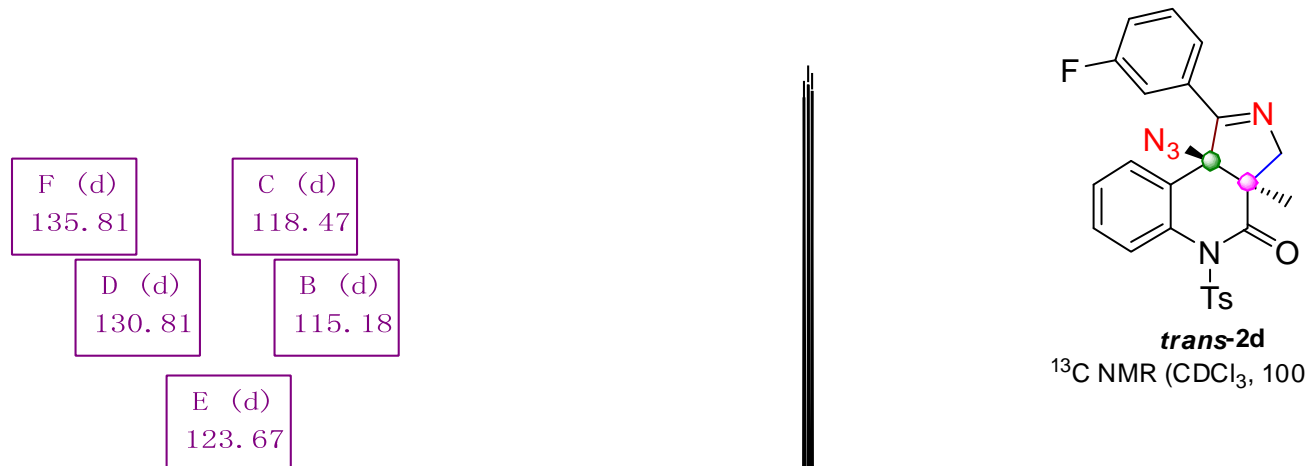

A (d)

123. 67

$\left.{ }^{13} \mathrm{C} \mathrm{NMR} \mathrm{(CDCl} 3,100 \mathrm{MHz}\right)$
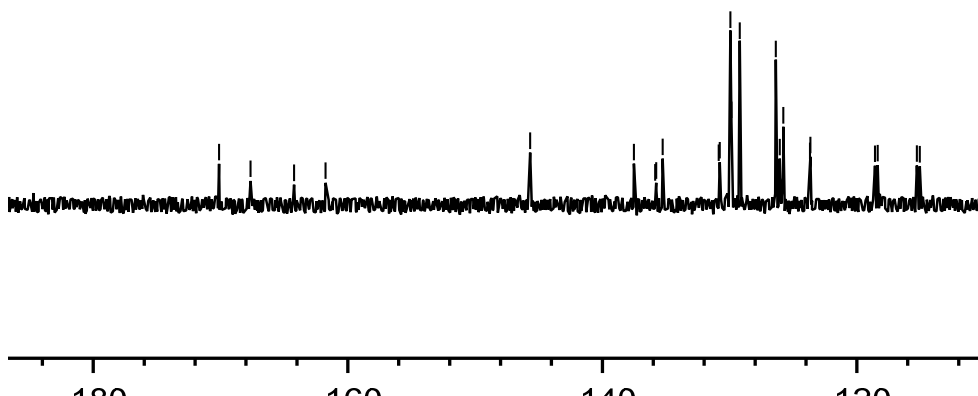

160

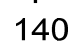

120

100

80

60

40

20 


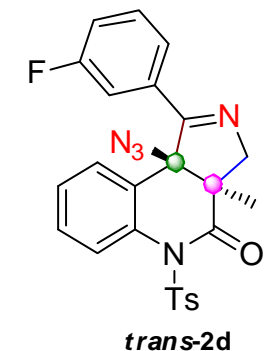

${ }^{19} \mathrm{~F} \mathrm{NMR}\left(\mathrm{CDCl}_{3}, 376 \mathrm{MHz}\right)$

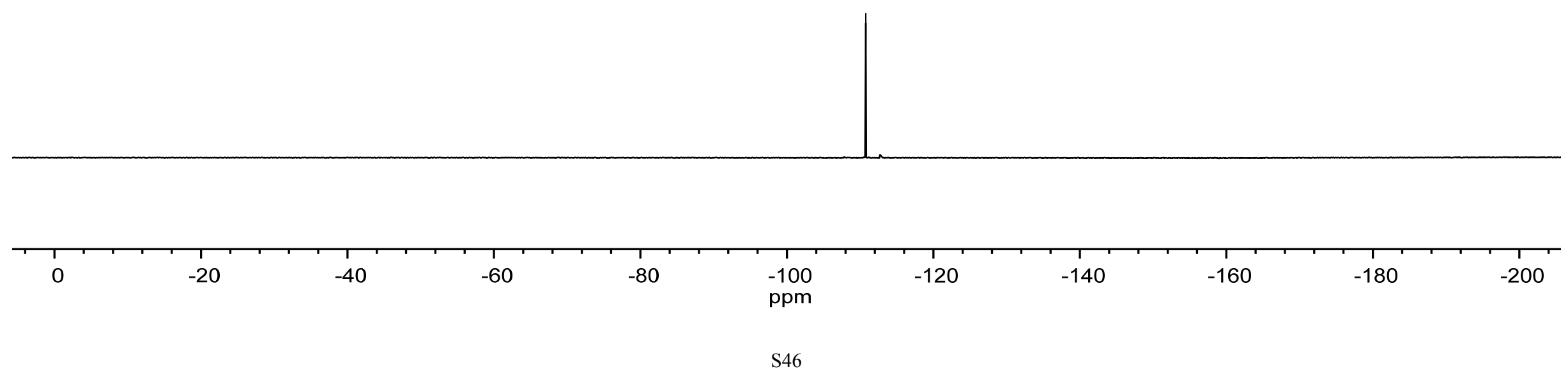




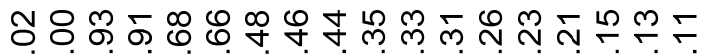

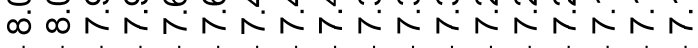

בـI
ஜ요 ○

में लंख

11
尔 者

น

$\stackrel{\infty}{\stackrel{\infty}{\circ}}$

8

i
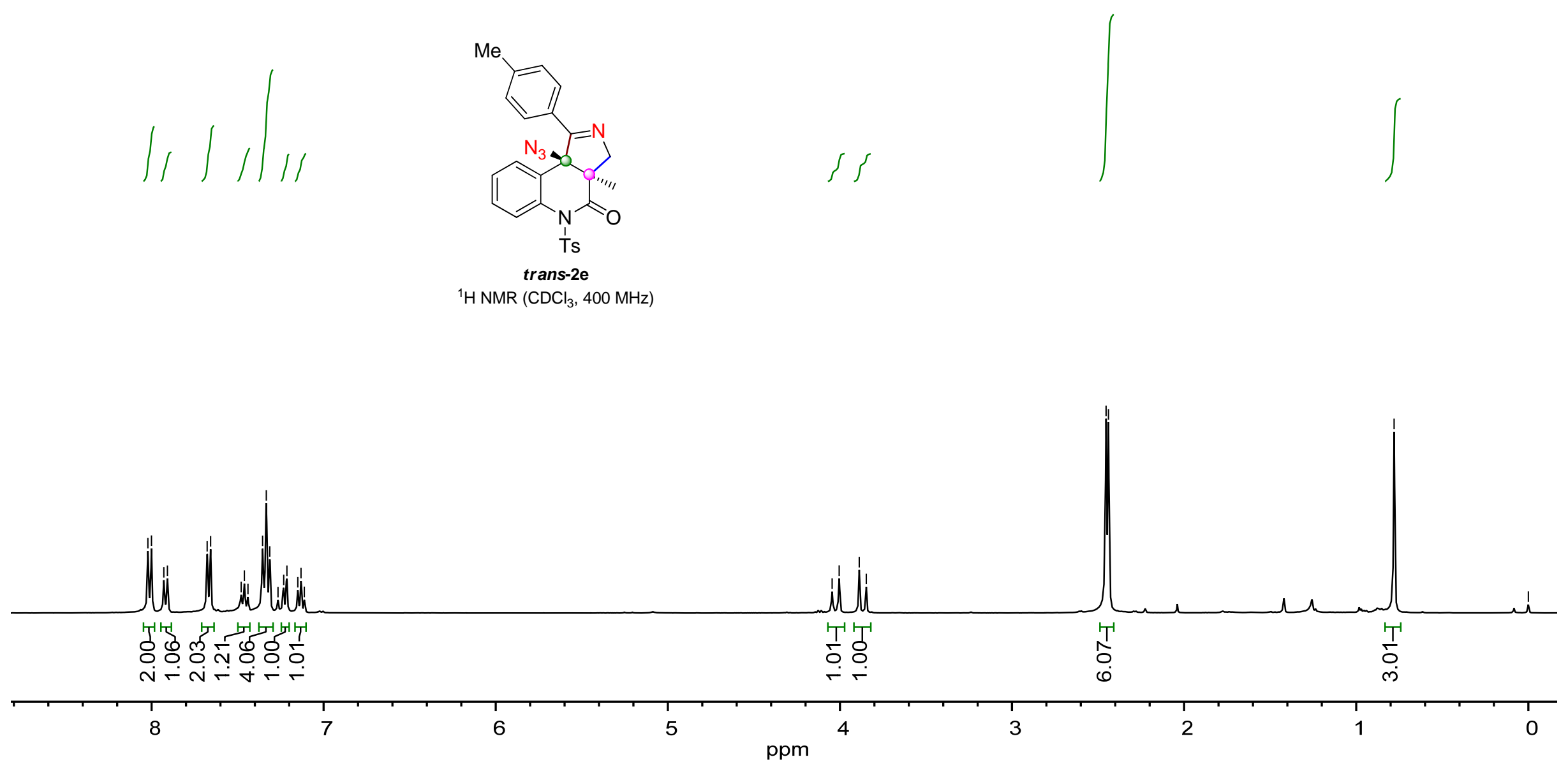
৫ํำ

䟻是

W

กิ

กำ

$\underbrace{2515}$

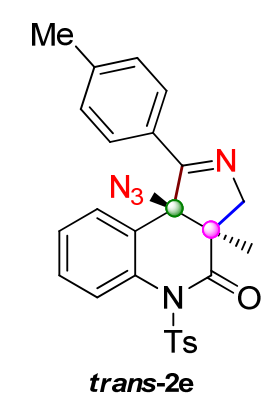

${ }^{13} \mathrm{C} \mathrm{NMR}\left(\mathrm{CDCl}_{3}, 100 \mathrm{MHz}\right)$
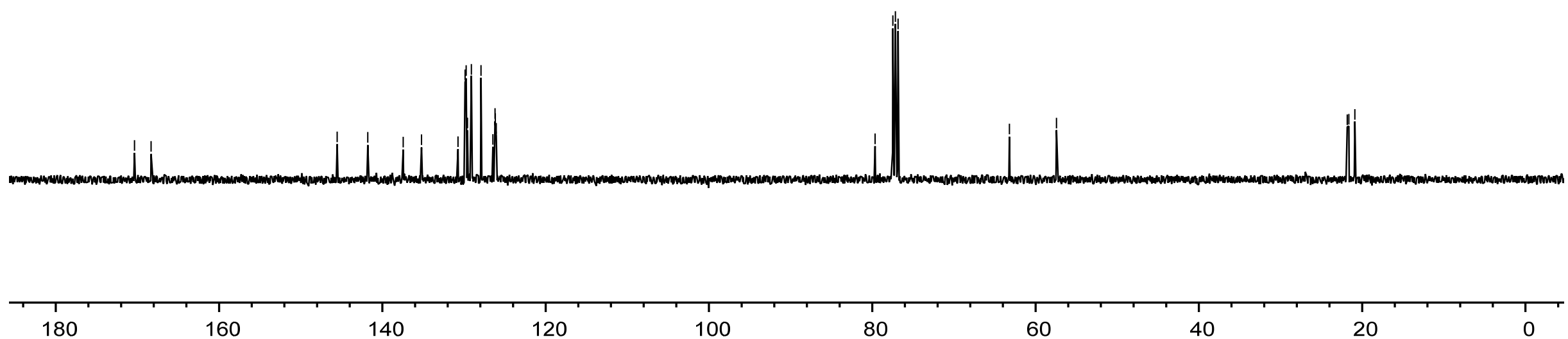

140

120

100

80

60

40 

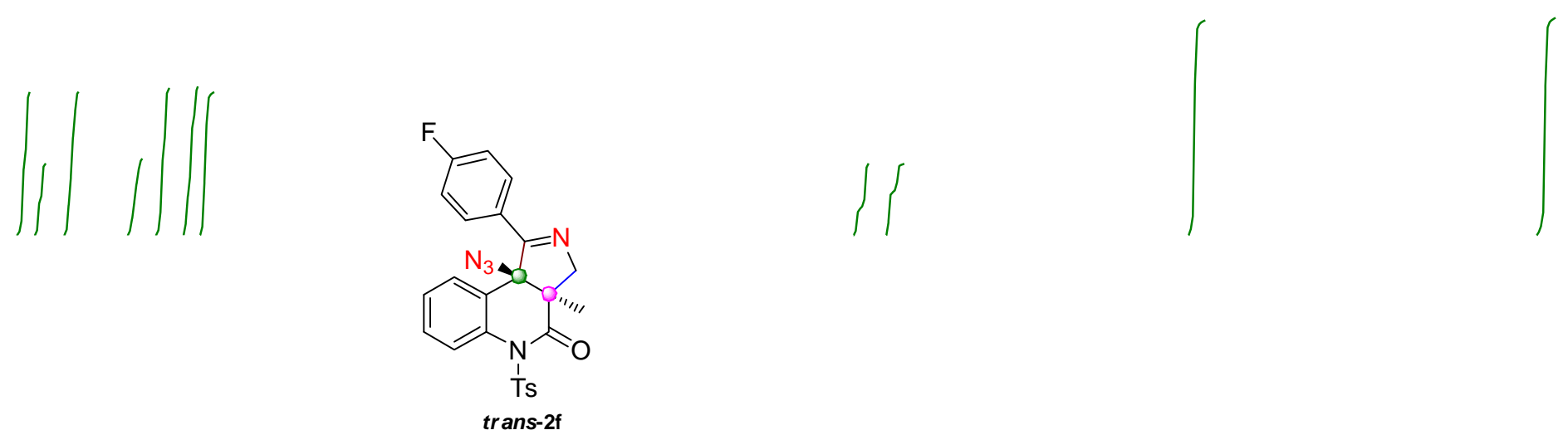

${ }^{1} \mathrm{H} \mathrm{NMR}\left(\mathrm{CDCl}_{3}, 400 \mathrm{MHz}\right)$

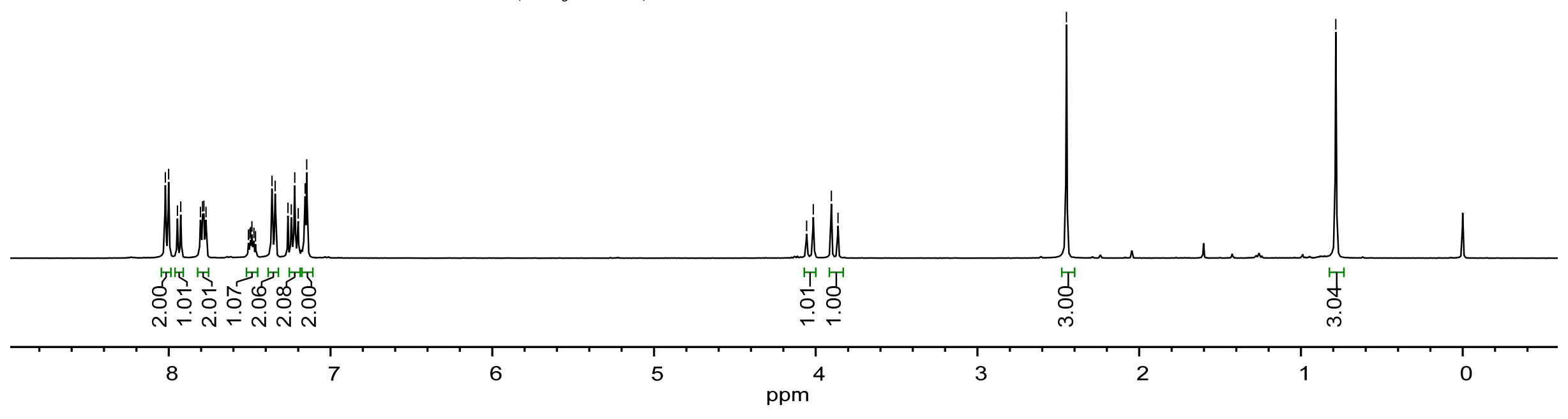




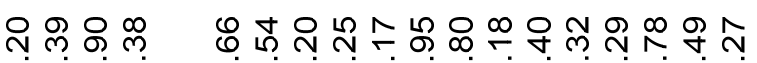

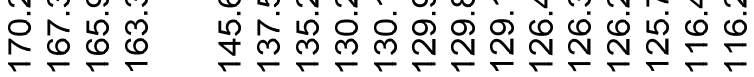

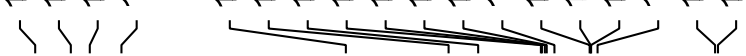

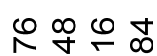

走卡员

\I

m.

ֻे

웜

$\bar{N} \bar{N}$

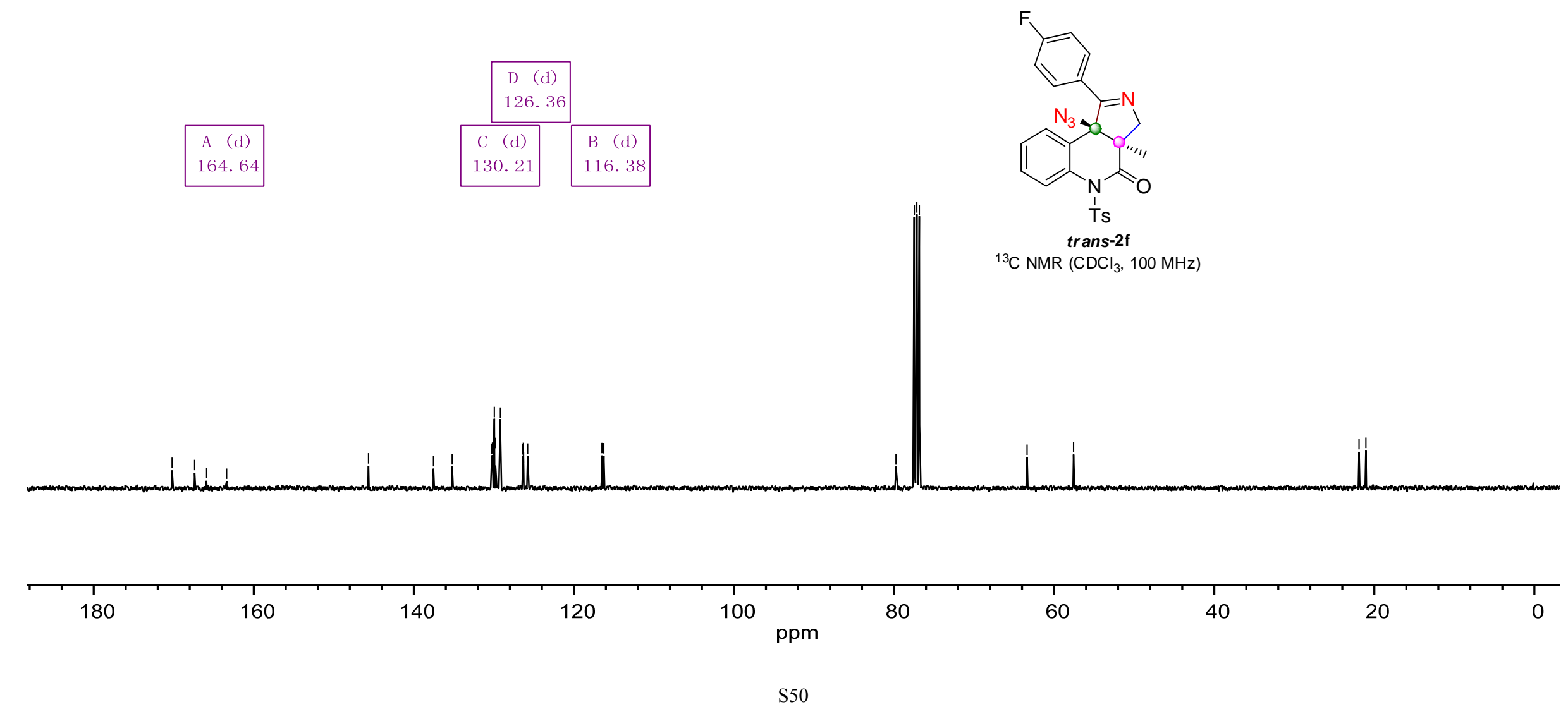




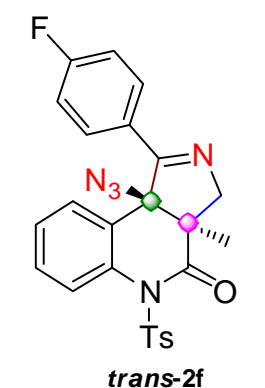

${ }^{19} \mathrm{~F} \mathrm{NMR}\left(\mathrm{CDCl}_{3}, 376 \mathrm{MHz}\right)$

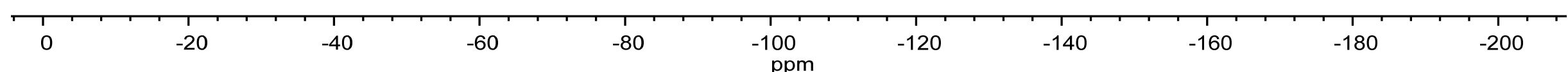




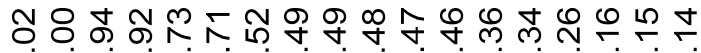

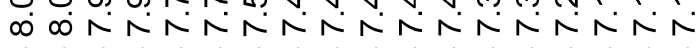

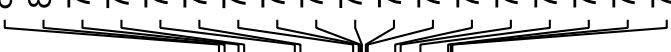

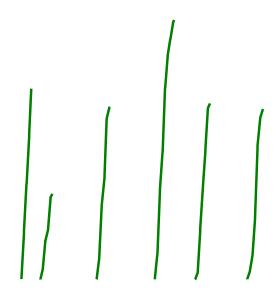

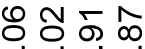

षं山ें

$+1$

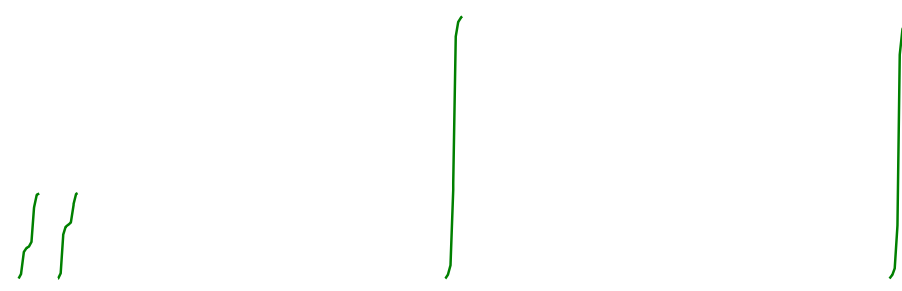

$\stackrel{8}{\stackrel{8}{~}}$

م

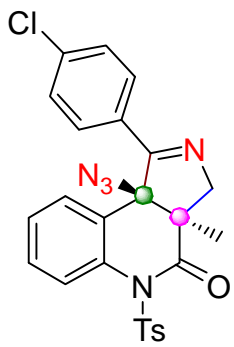

ᄋำ

i

trans-2g

${ }^{1} \mathrm{H} \mathrm{NMR}\left(\mathrm{CDCl}_{3}, 400 \mathrm{MHz}\right)$

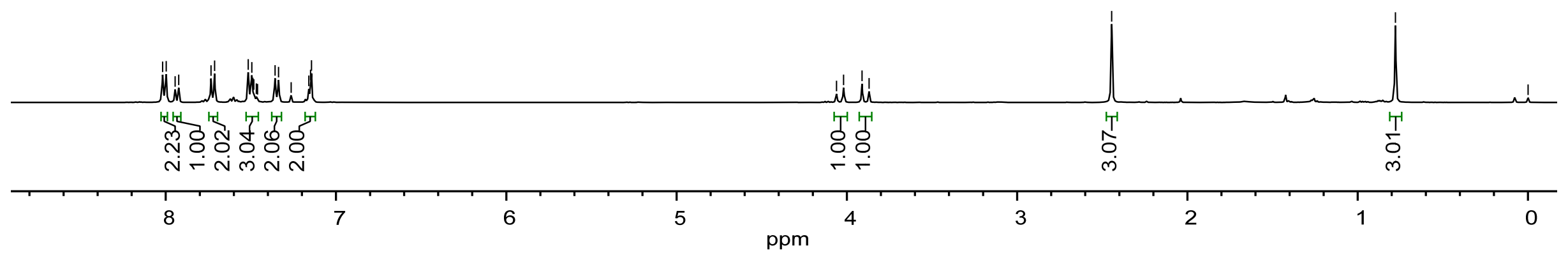




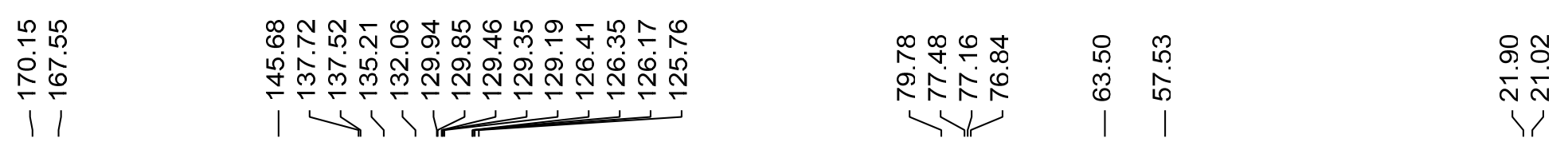

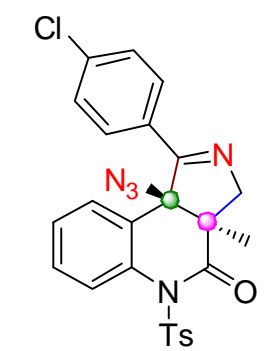

trans-2g

${ }^{13} \mathrm{C}$ NMR $\left(\mathrm{CDCl}_{3}, 100 \mathrm{MHz}\right)$

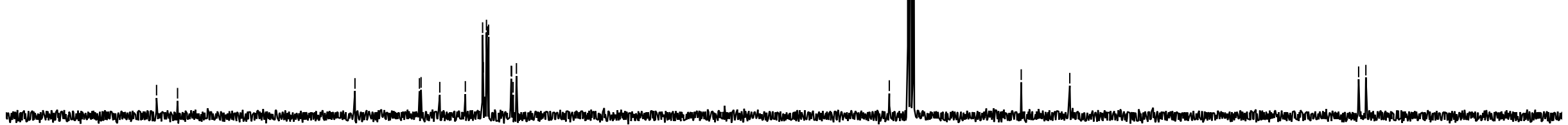

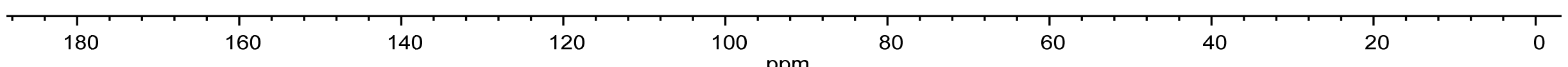



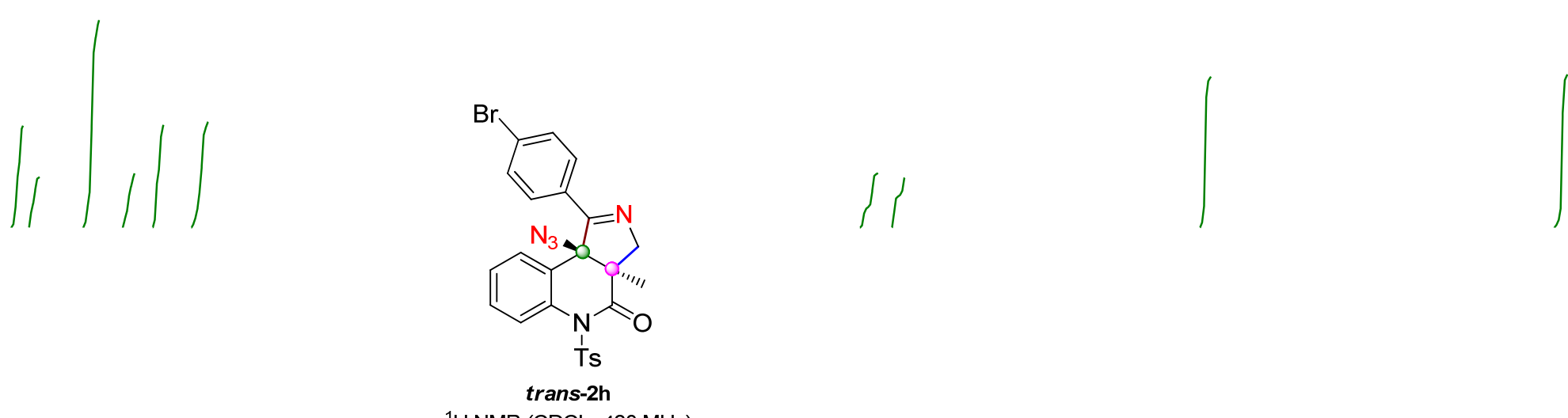

${ }^{1} \mathrm{HNMR}\left(\mathrm{CDCl}_{3}, 400 \mathrm{MHz}\right)$

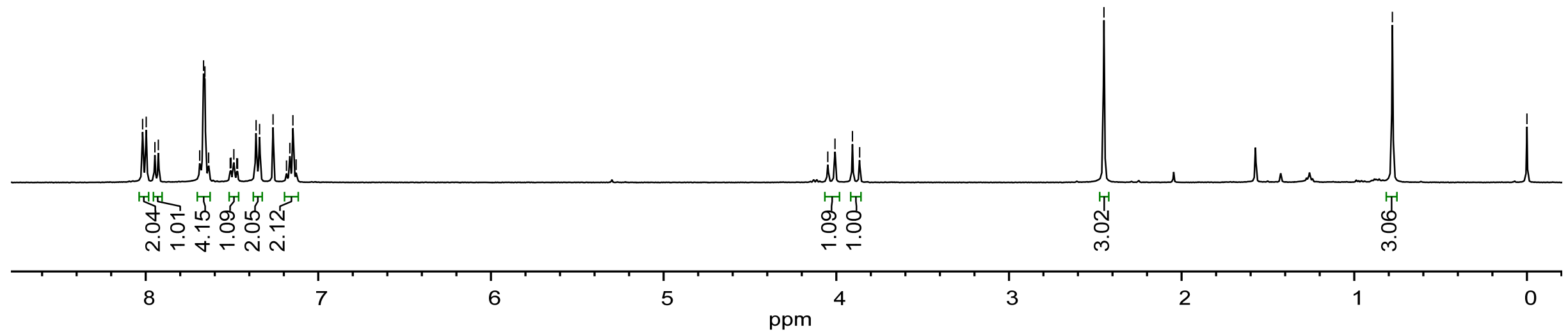




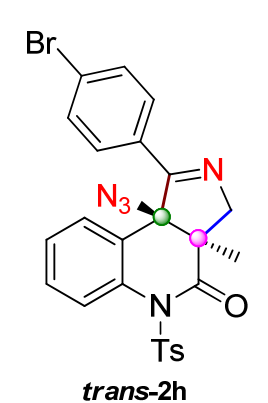

$\left.\left.{ }^{13} \mathrm{C} \mathrm{NMR} \mathrm{(CDCl}\right)_{3}, 100 \mathrm{MHz}\right)$

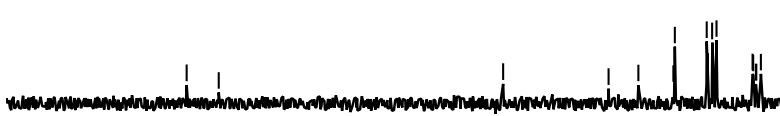


ช

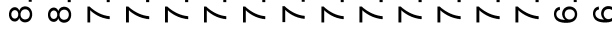

(1)

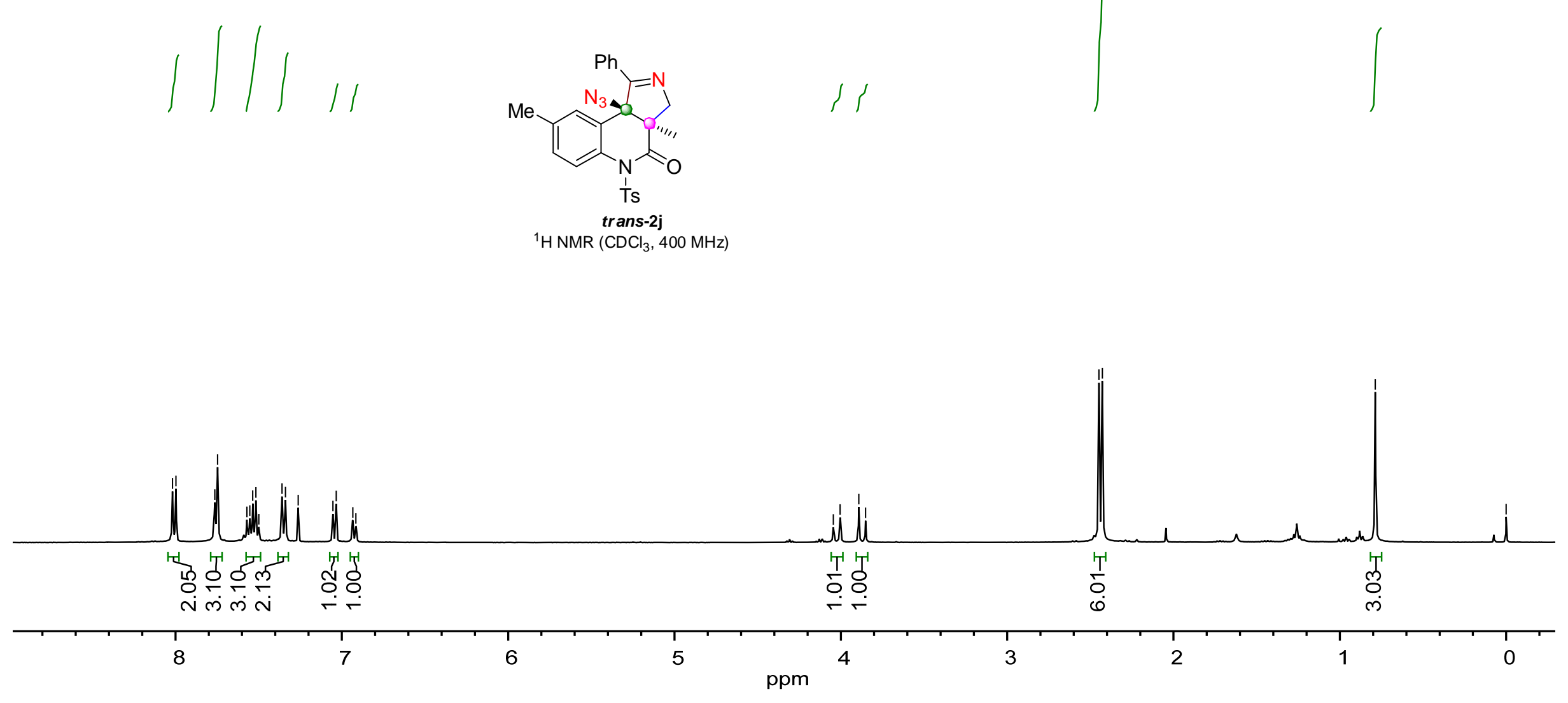

ப요

$+\dot{+} \dot{0}$

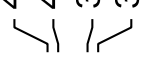

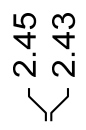

0
0
0

웅

1 


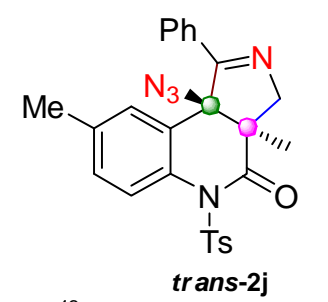

${ }^{13} \mathrm{C}$ NMR $\left(\mathrm{CDCl}_{3}, 100 \mathrm{MHz}\right)$
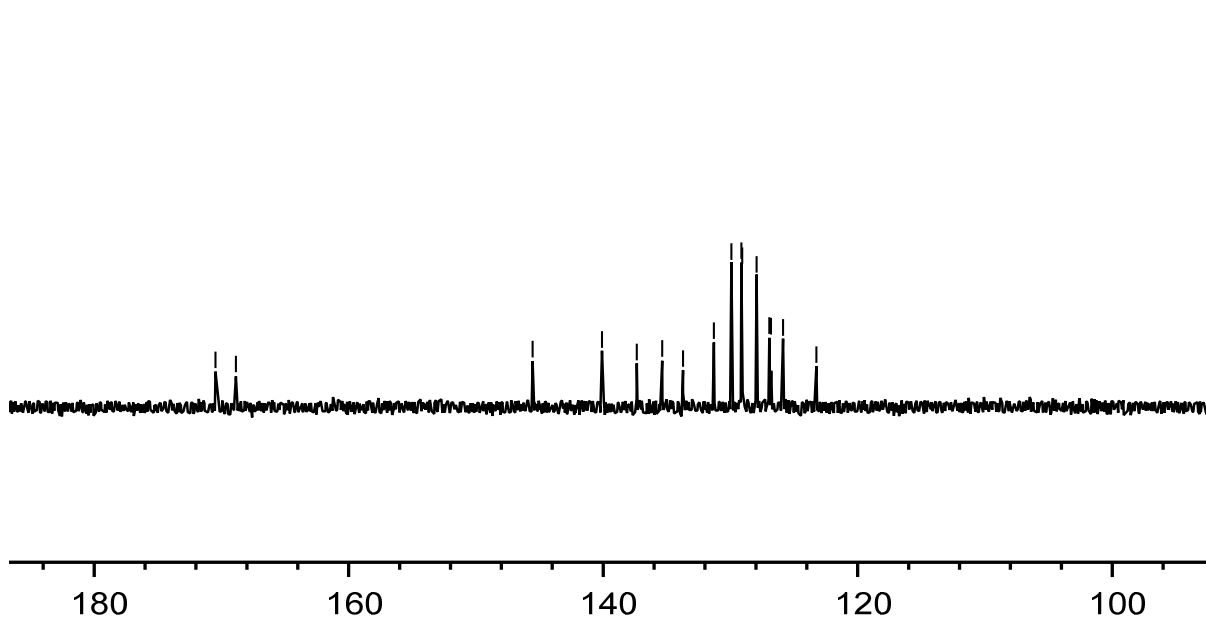

120

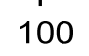

80

60

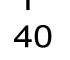




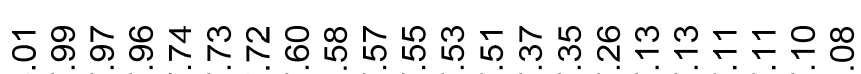

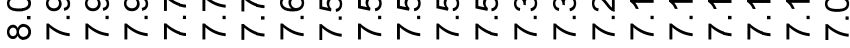

$\longrightarrow$

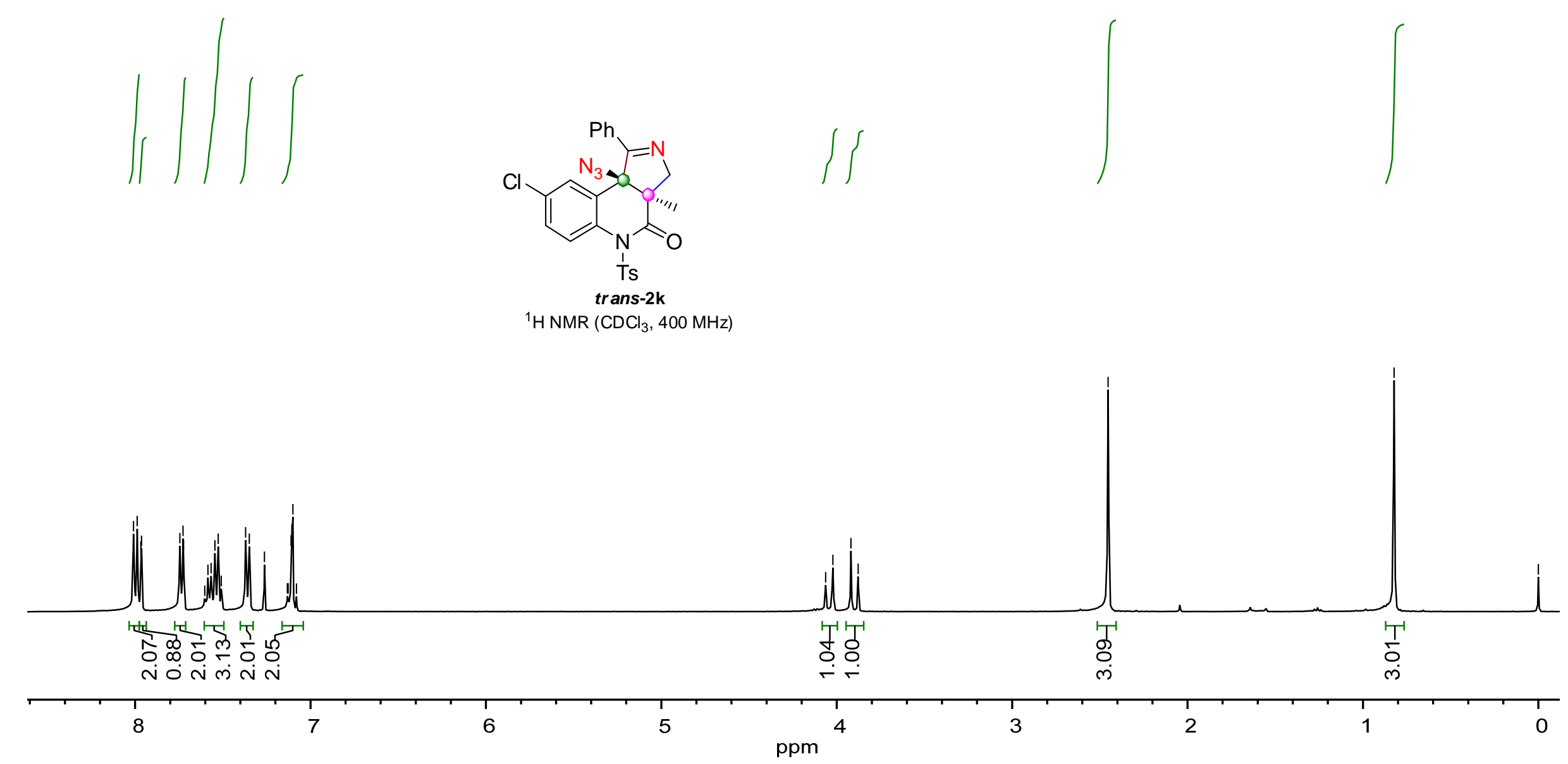

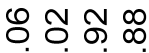

मेंल

प1
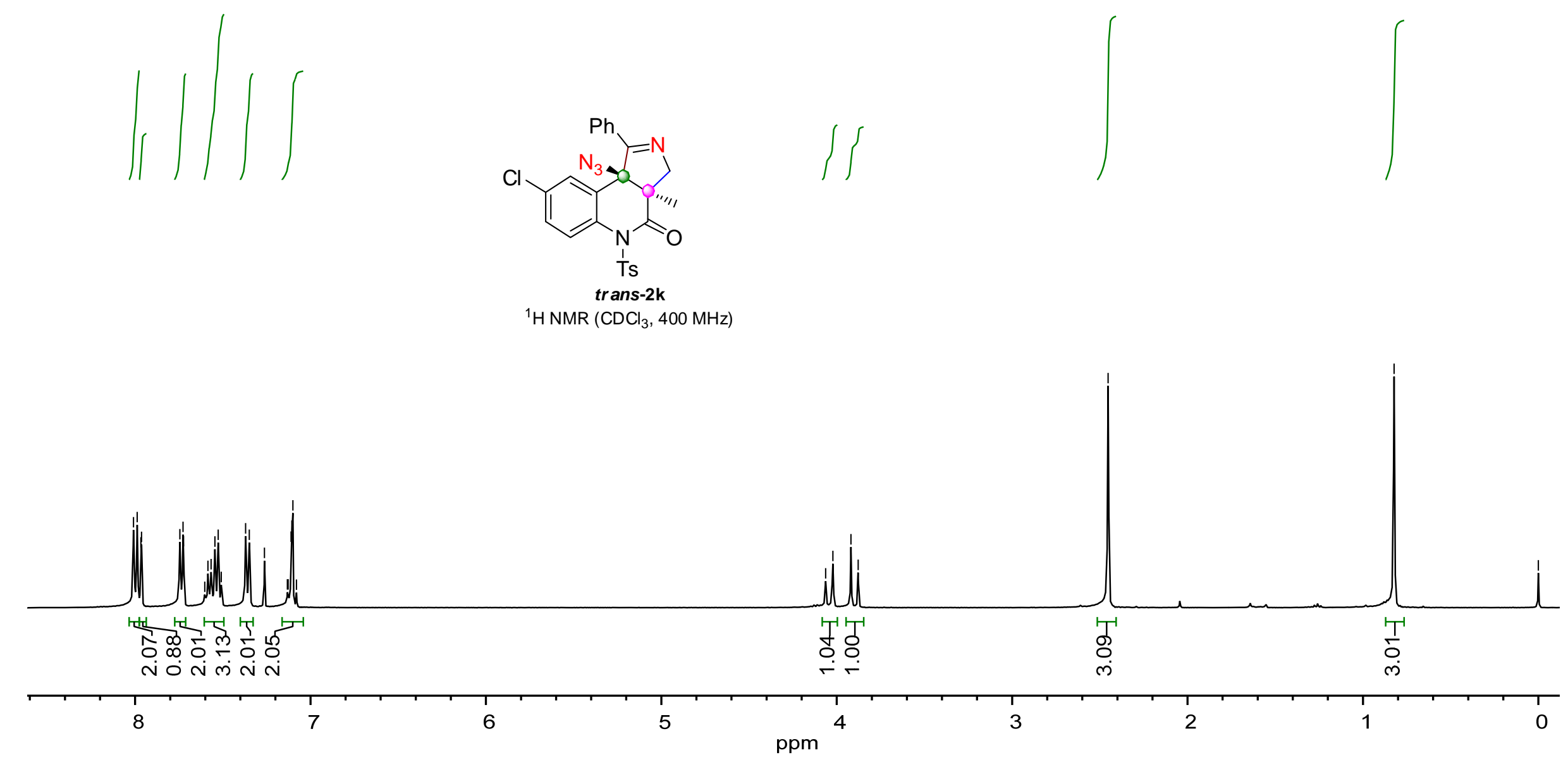

${ }^{1} \mathrm{H} \mathrm{NMR}\left(\mathrm{CDCl}_{3}, 400 \mathrm{MHz}\right)$ 


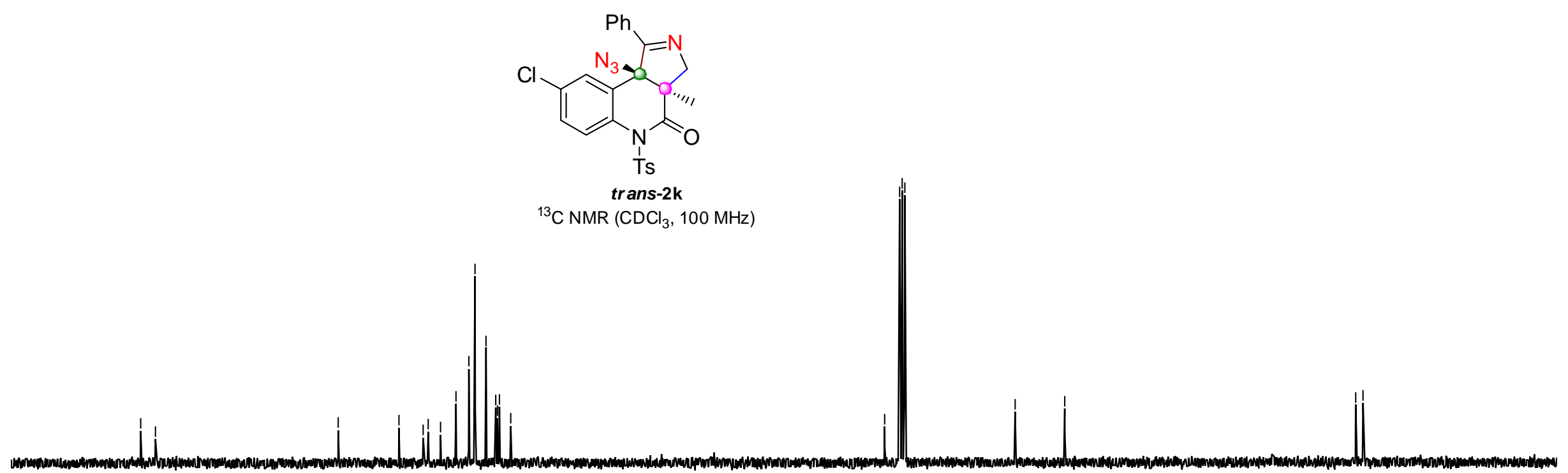



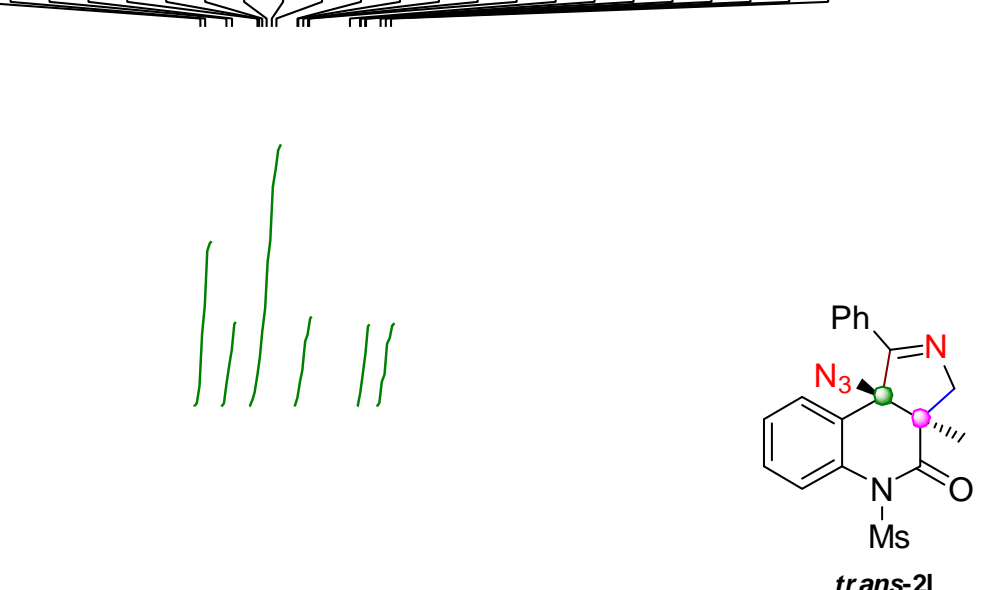

${ }^{1} \mathrm{H} \mathrm{NMR}\left(\mathrm{CDCl}_{3}, 400 \mathrm{MHz}\right)$
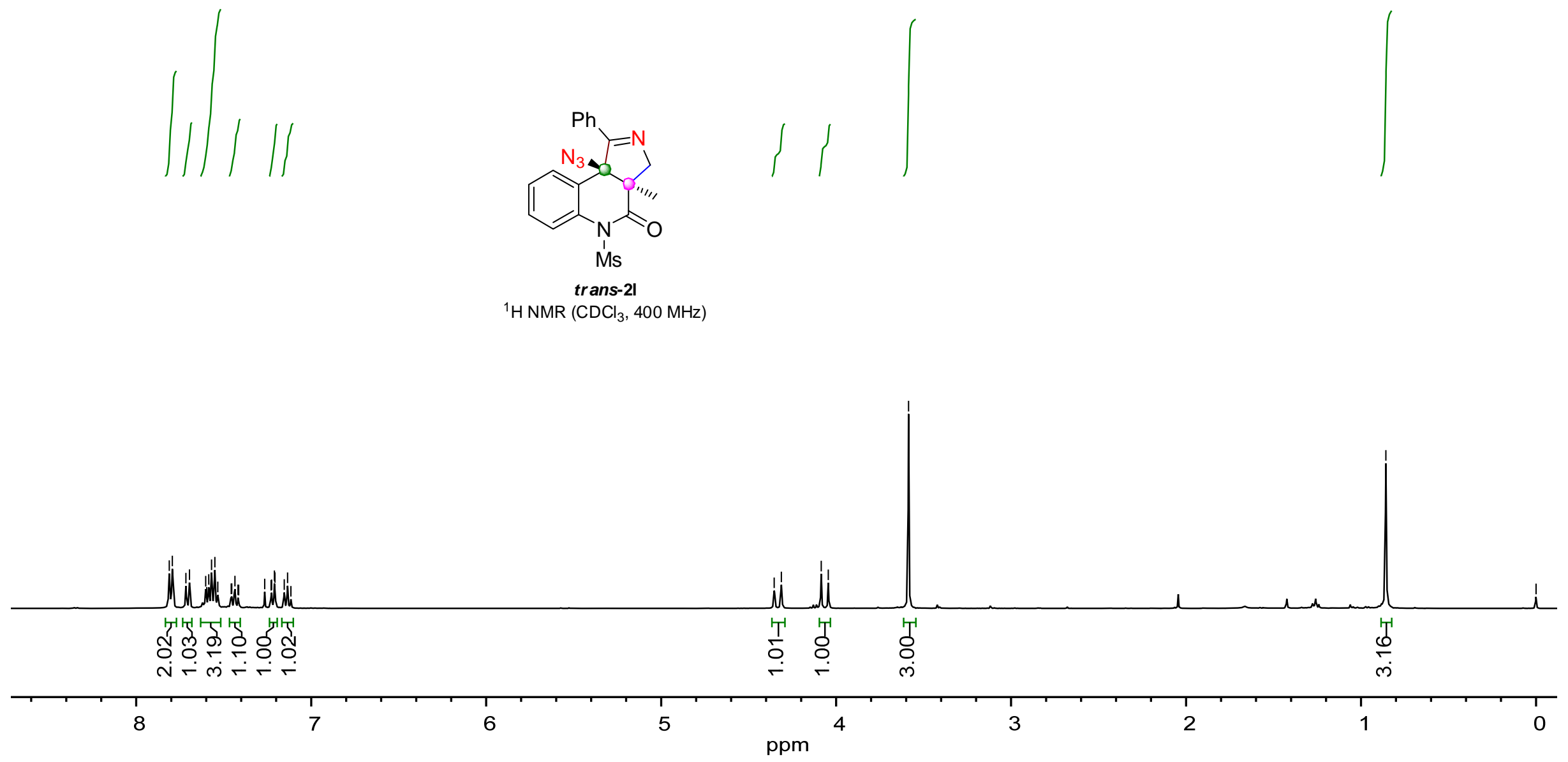
$\infty$

동

|

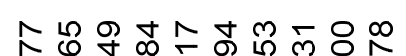

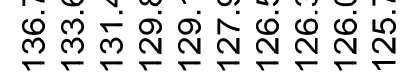

=

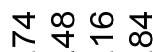

於走通

\ل1

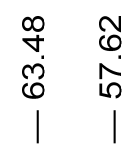

$\stackrel{\infty}{\stackrel{\infty}{N}}$

@:
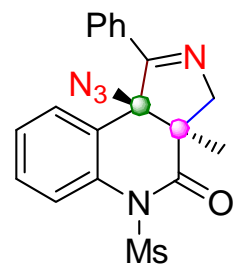

trans-2

${ }^{13} \mathrm{C} \mathrm{NMR}\left(\mathrm{CDCl}_{3}, 100 \mathrm{MHz}\right)$

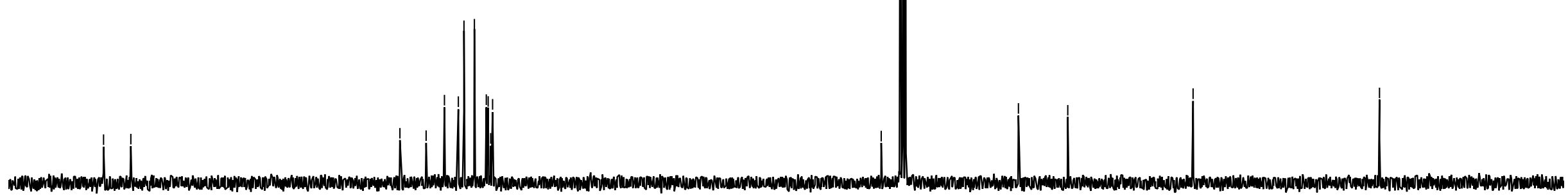

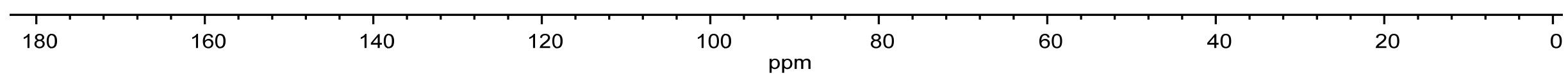



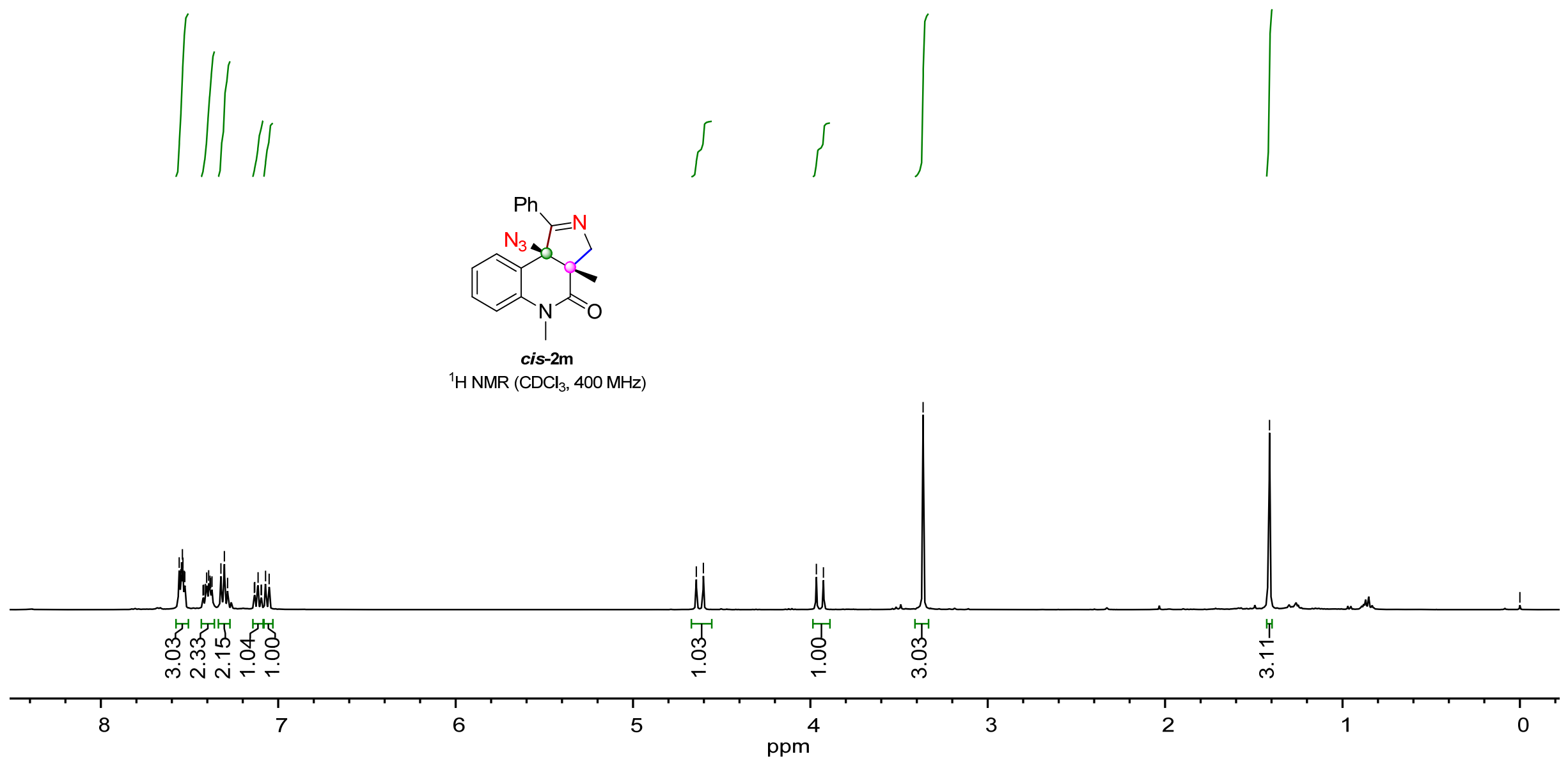


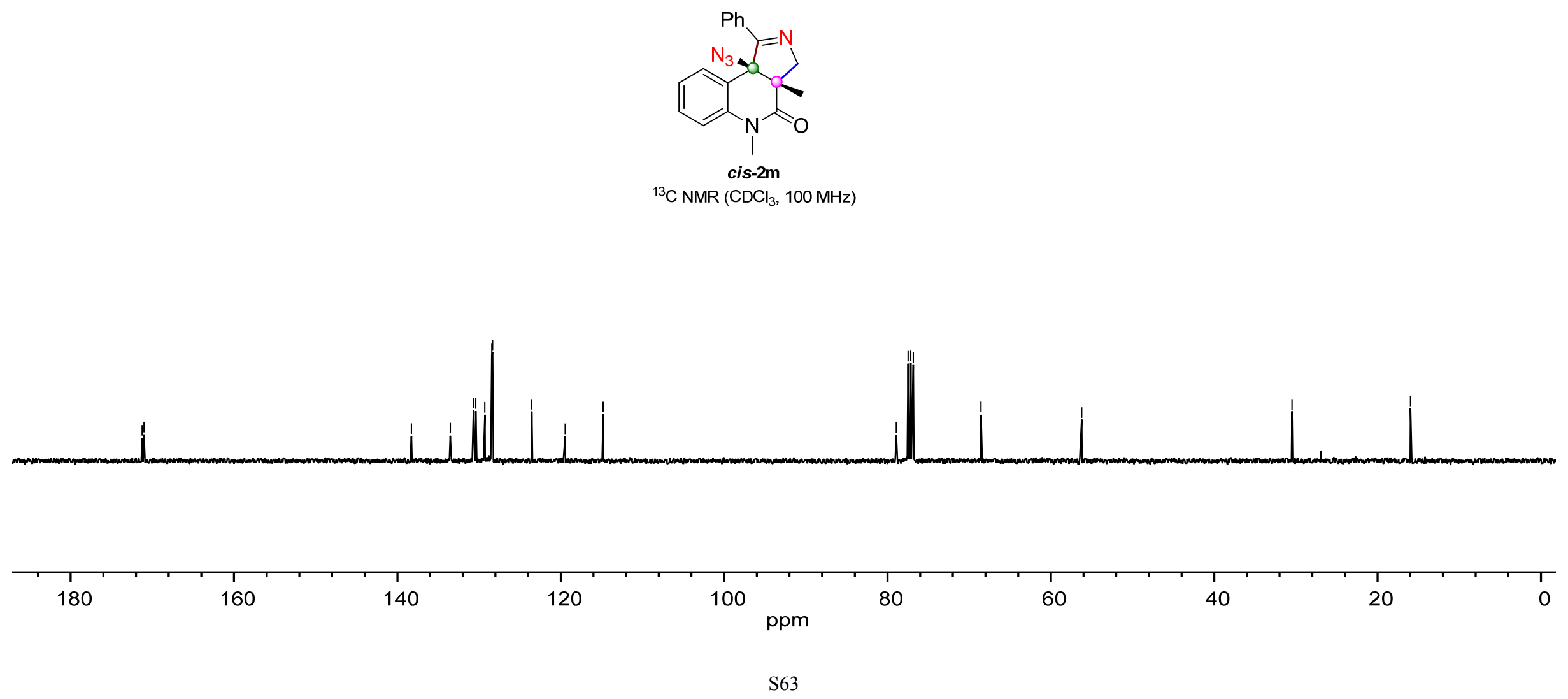



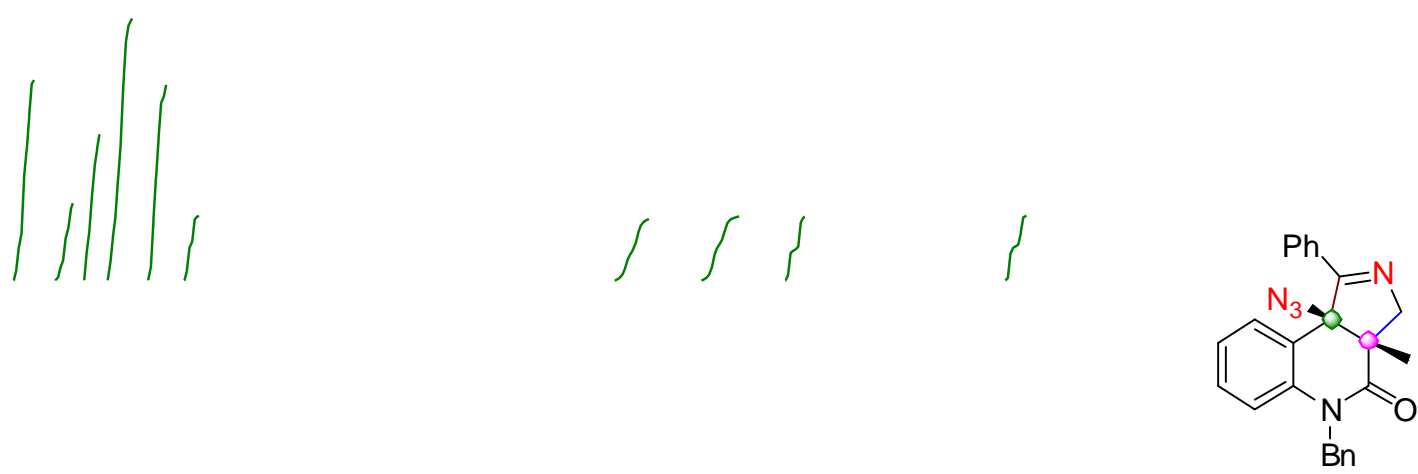

${ }^{1} \mathrm{H} \mathrm{NMR}\left(\mathrm{CDCl}_{3}, 400 \mathrm{MHz}\right)$

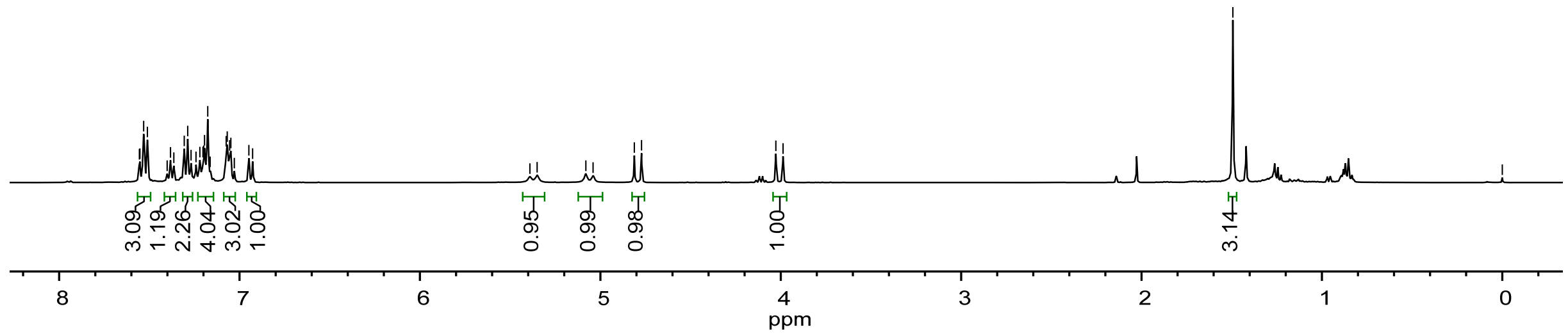




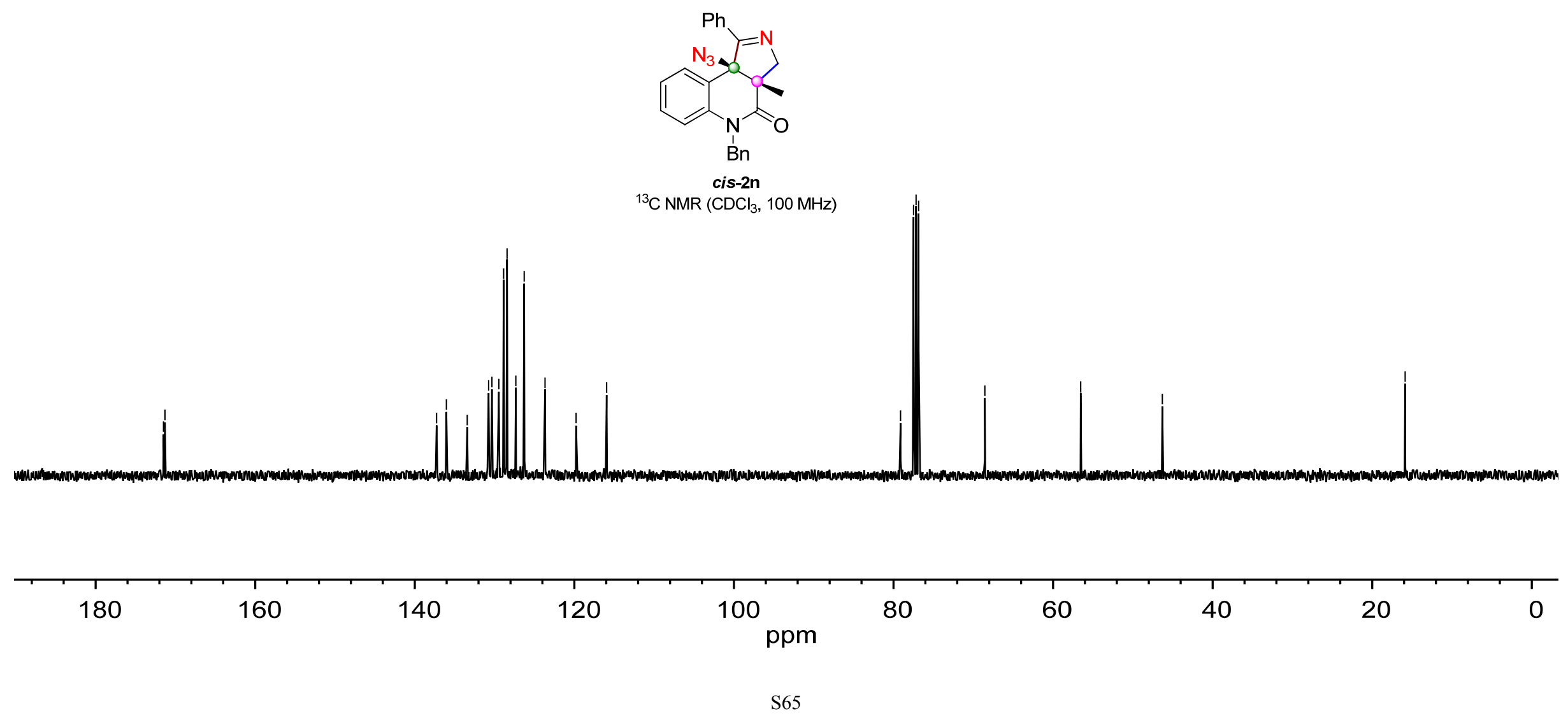


(n)
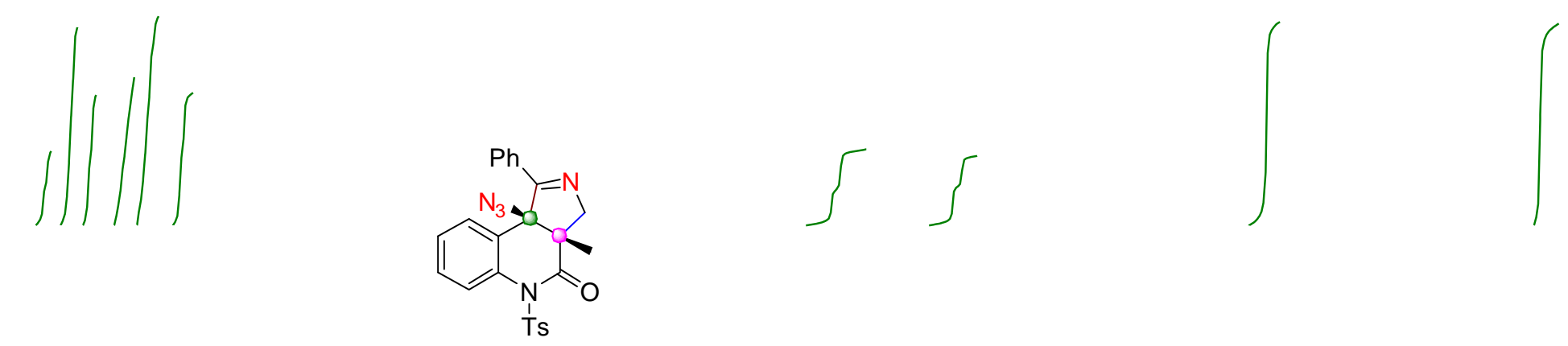

${ }^{1} \mathrm{H} \mathrm{NMR}\left(\mathrm{CDCl}_{3}, 400 \mathrm{MHz}\right)$

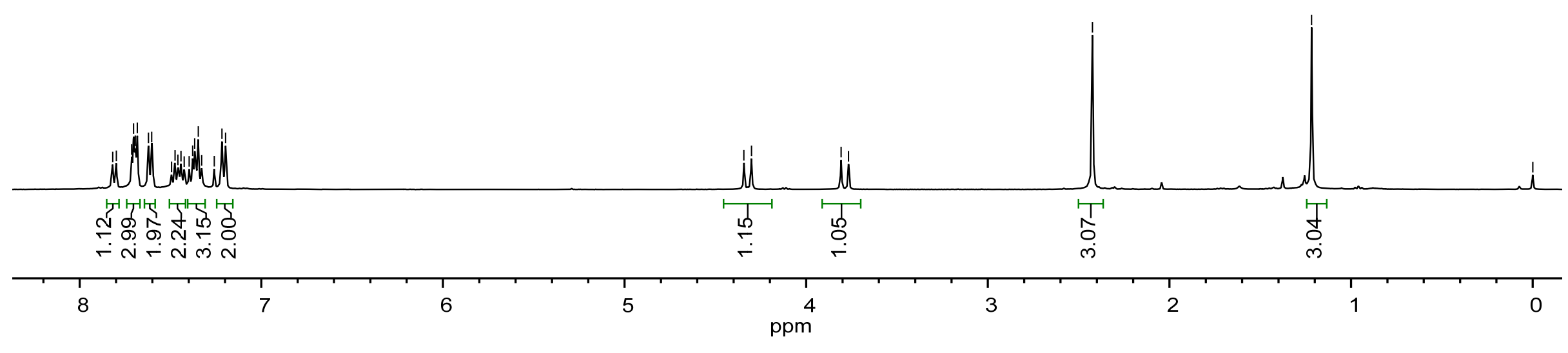



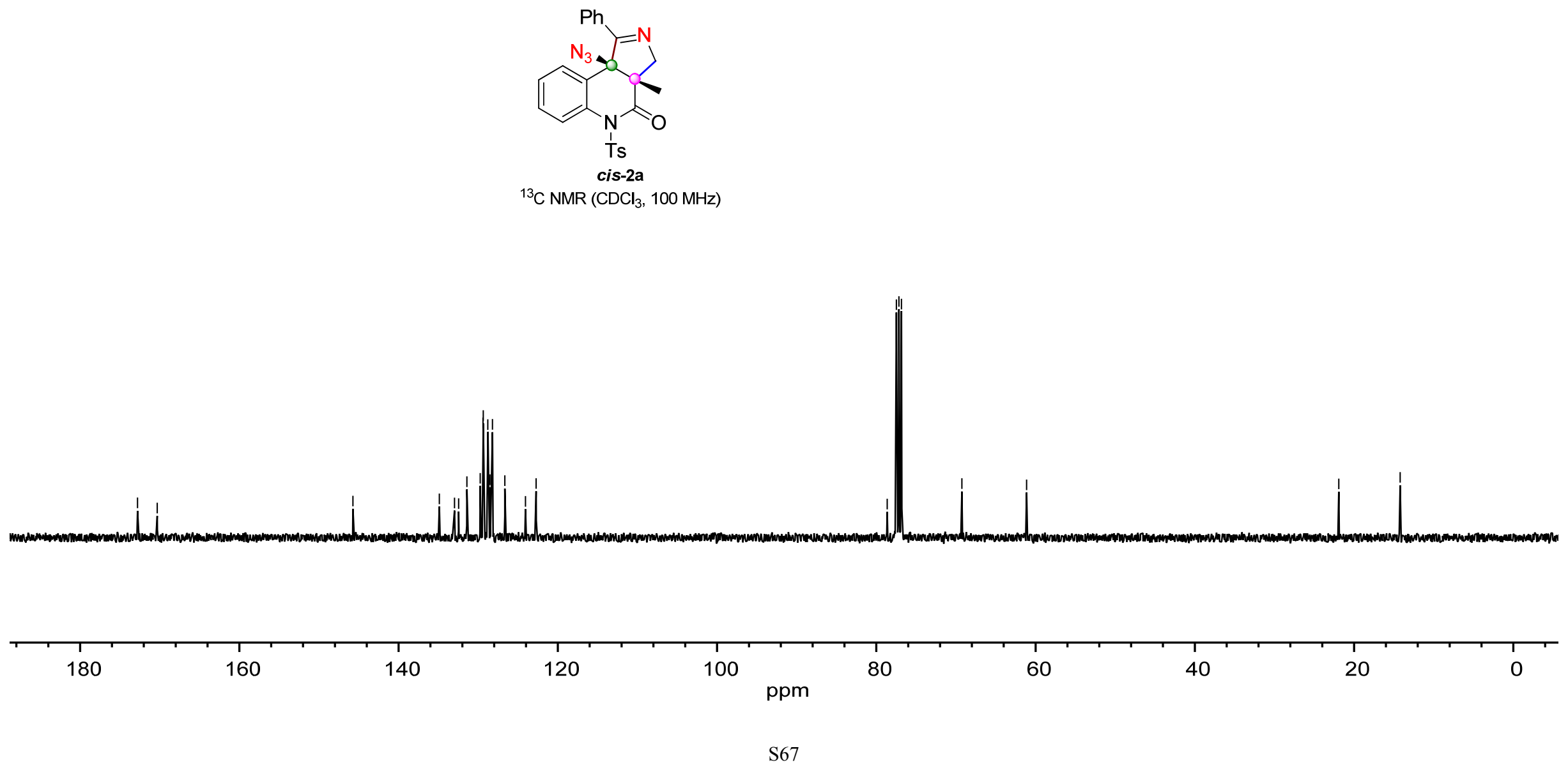


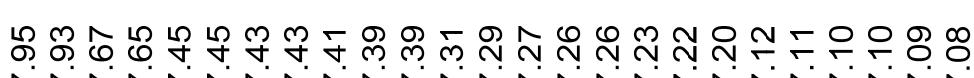

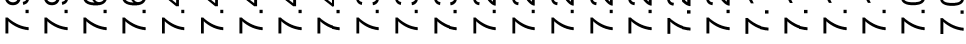
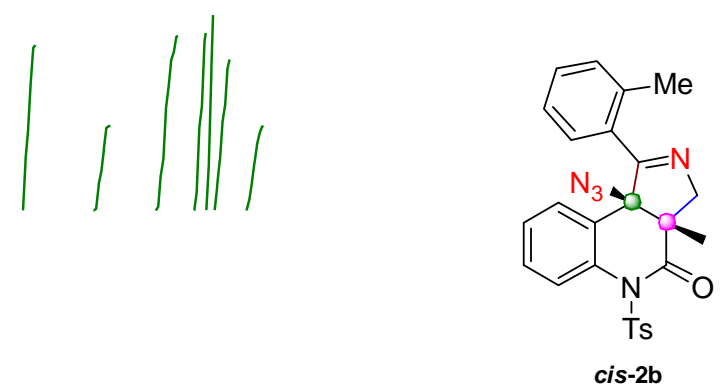

${ }^{1} \mathrm{H} \mathrm{NMR}\left(\mathrm{CDCl}_{3}, 400 \mathrm{MHz}\right)$

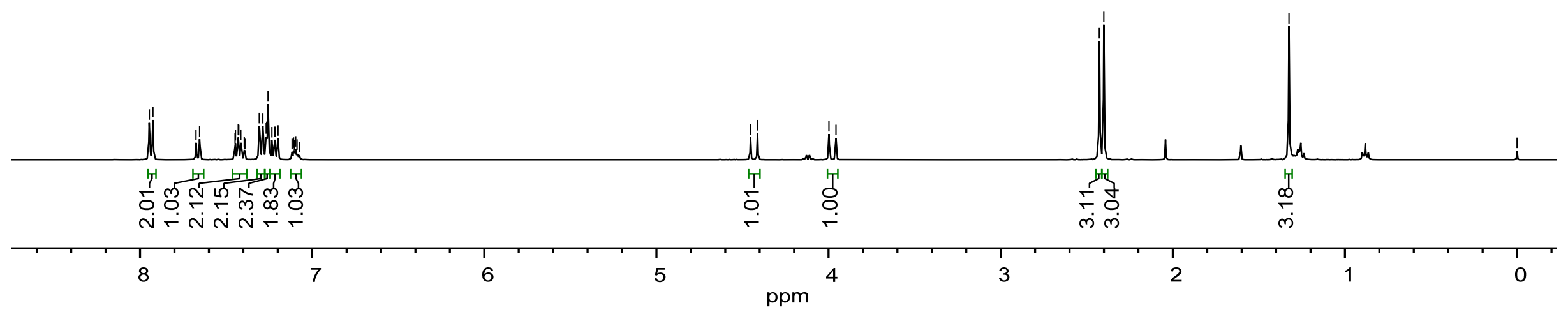

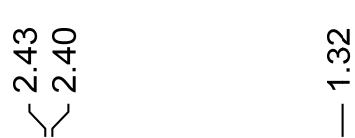

8

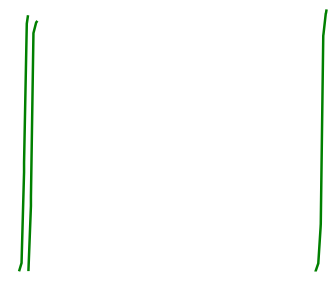

$$
\text { . }
$$




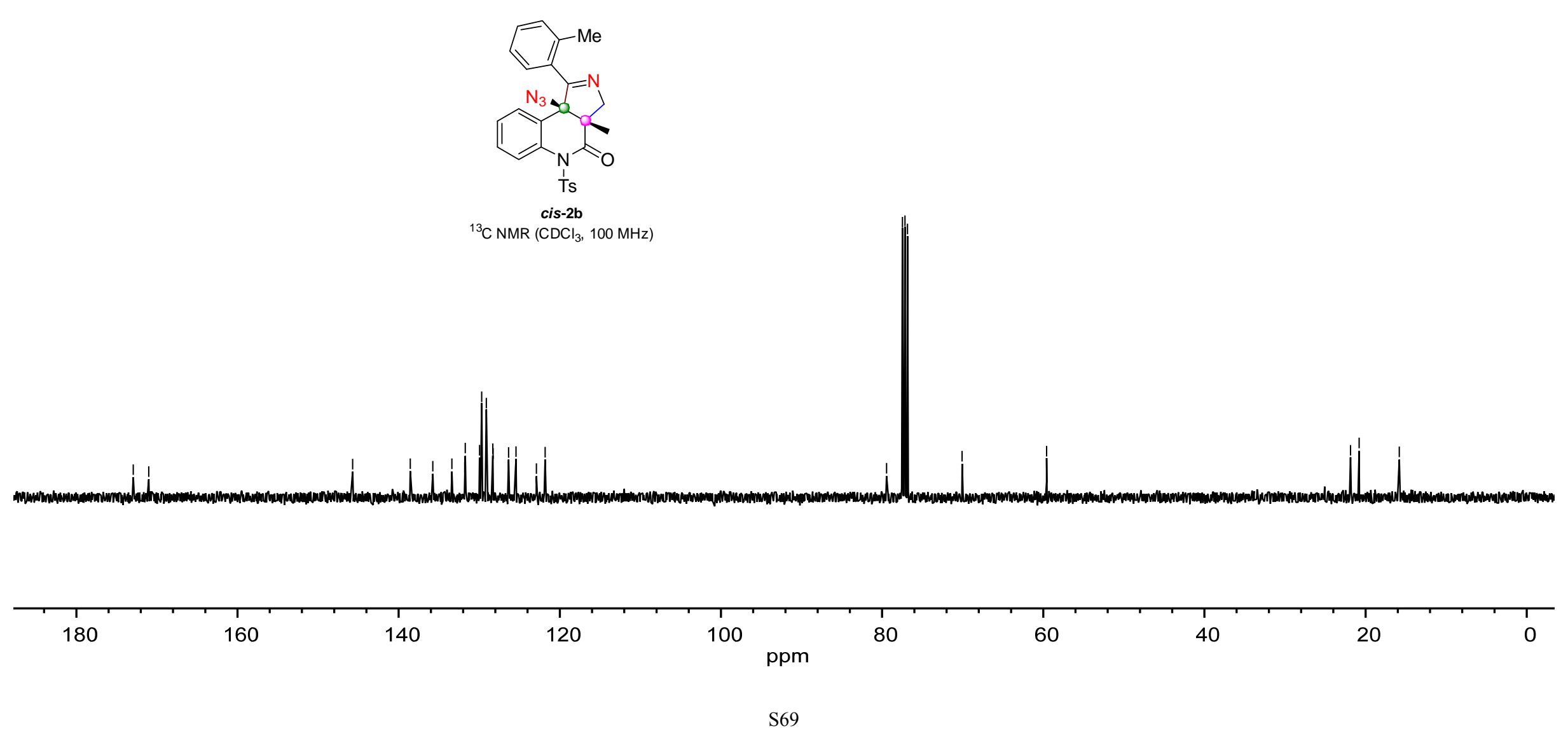




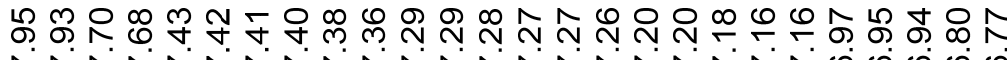

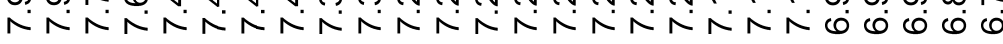

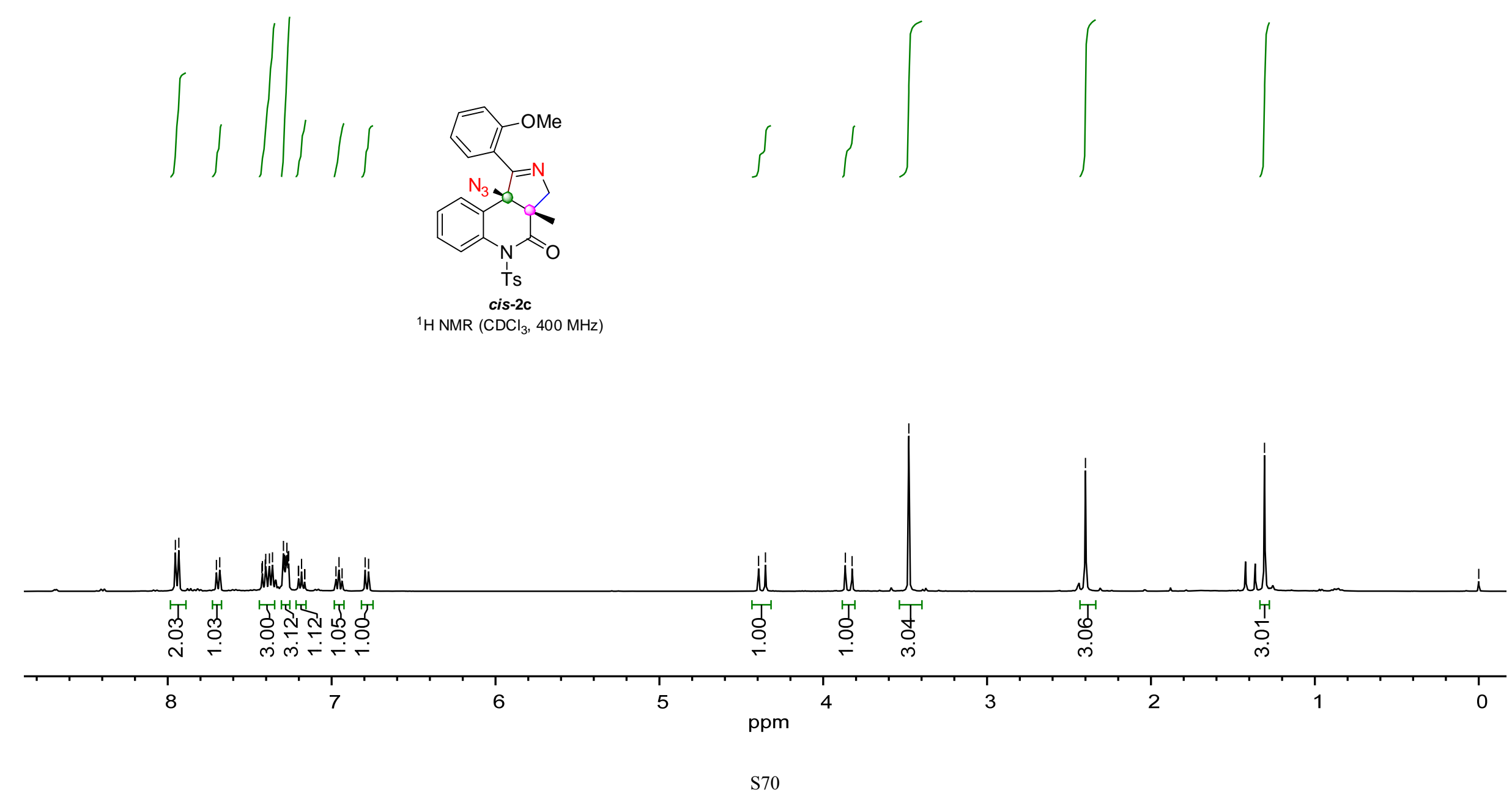

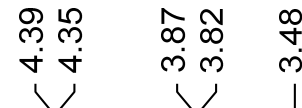

운

๓े

i

8

i 


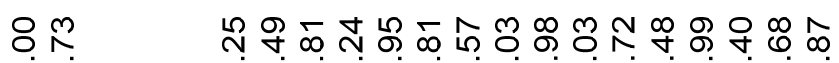

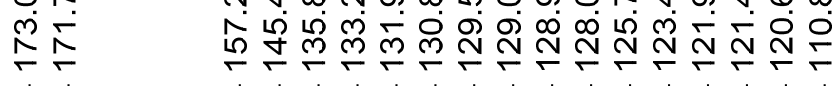

|

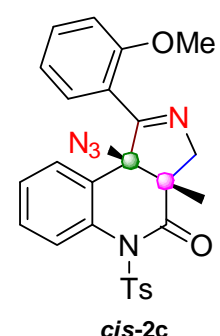

${ }^{13} \mathrm{C} \mathrm{NMR}\left(\mathrm{CDCl}_{3}, 100 \mathrm{MHz}\right)$

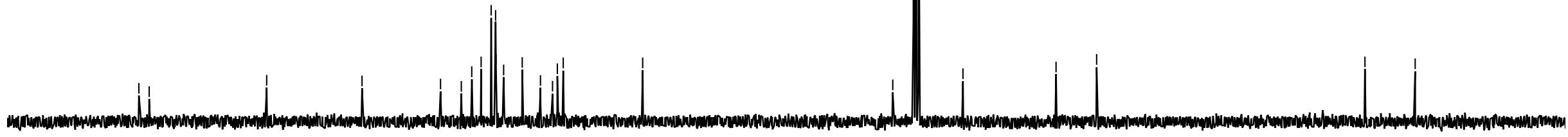

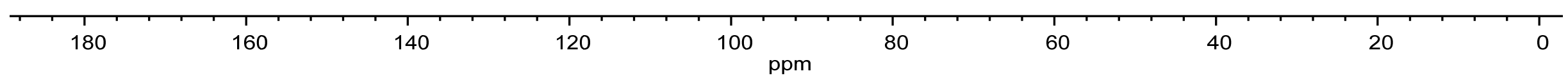



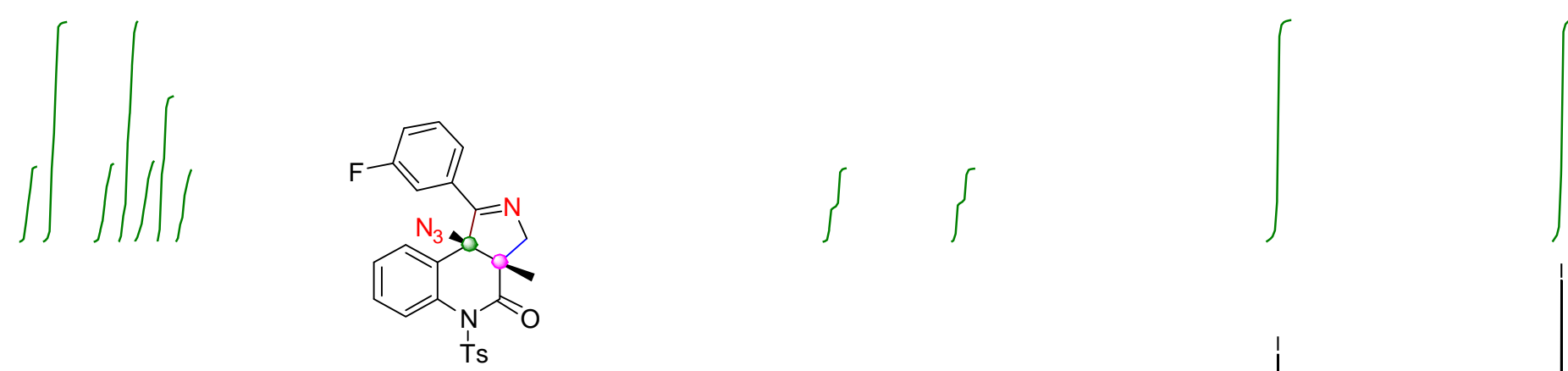

${ }_{1} \mathrm{H} \mathrm{NMR}\left(\mathrm{CDCl}_{3}, 400 \mathrm{MHz}\right)$
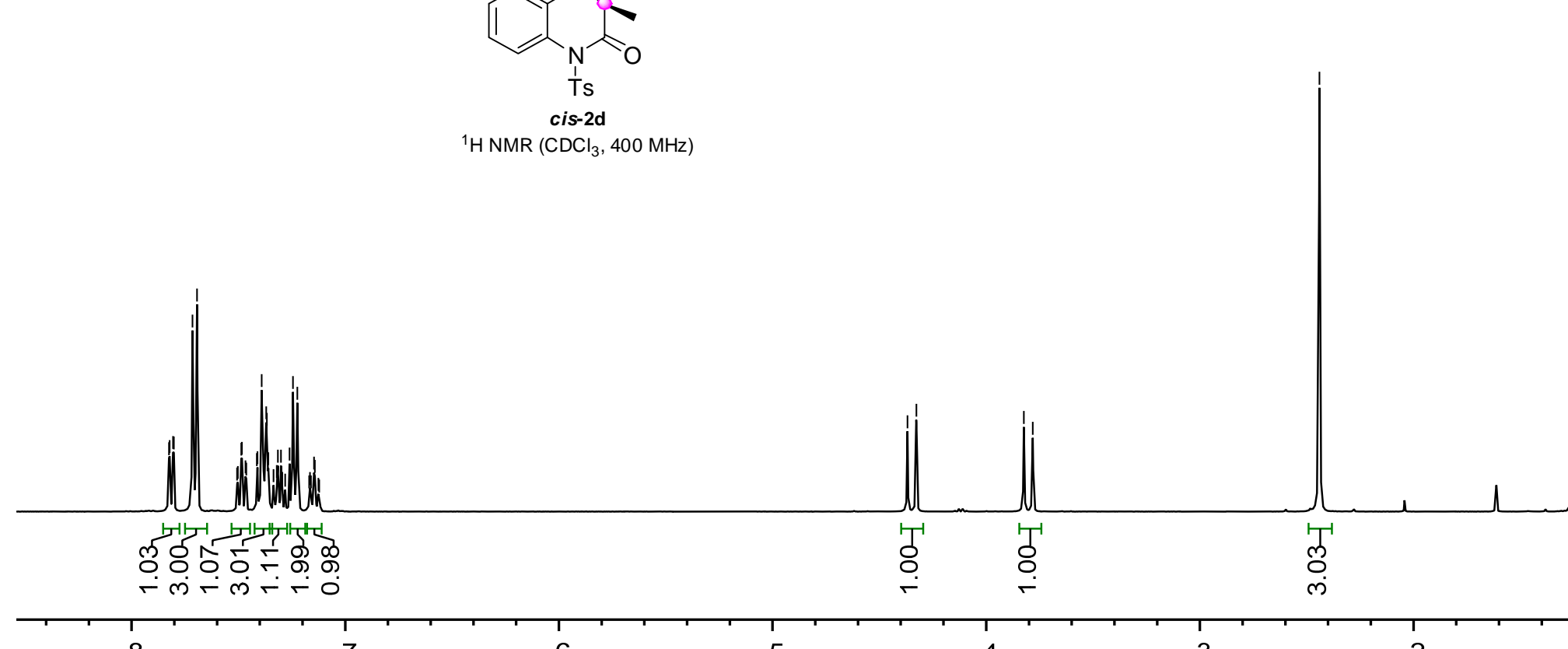

8

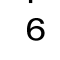

5

ppm

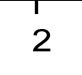


๒ิ

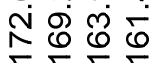

ij

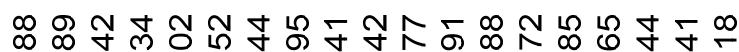

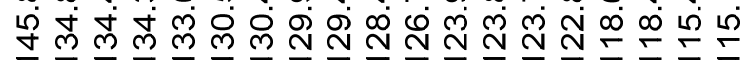

皮

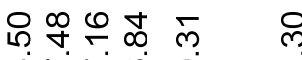

余卡官

।
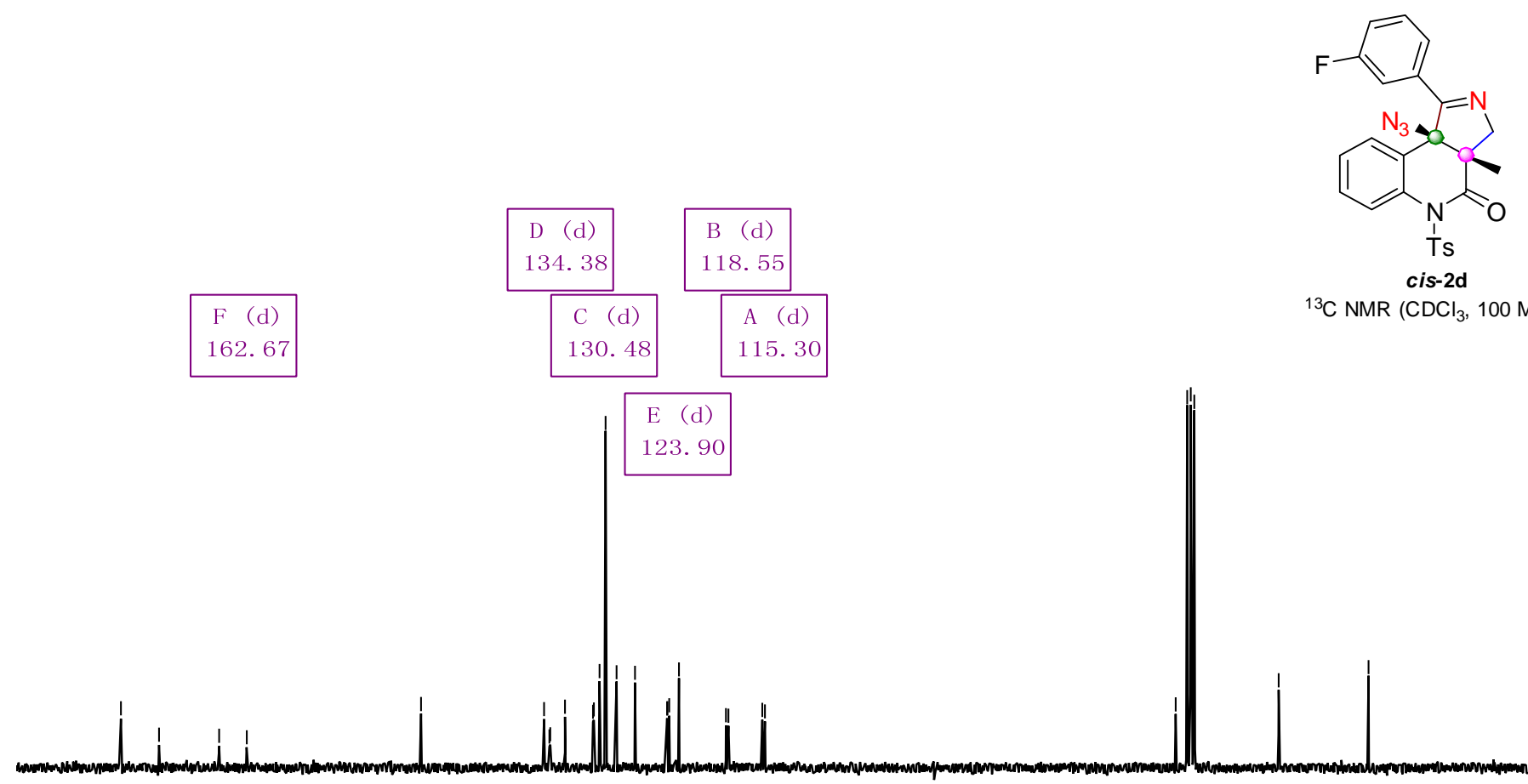

${ }^{13} \mathrm{C} \mathrm{NMR}\left(\mathrm{CDCl}_{3}, 100 \mathrm{MHz}\right)$
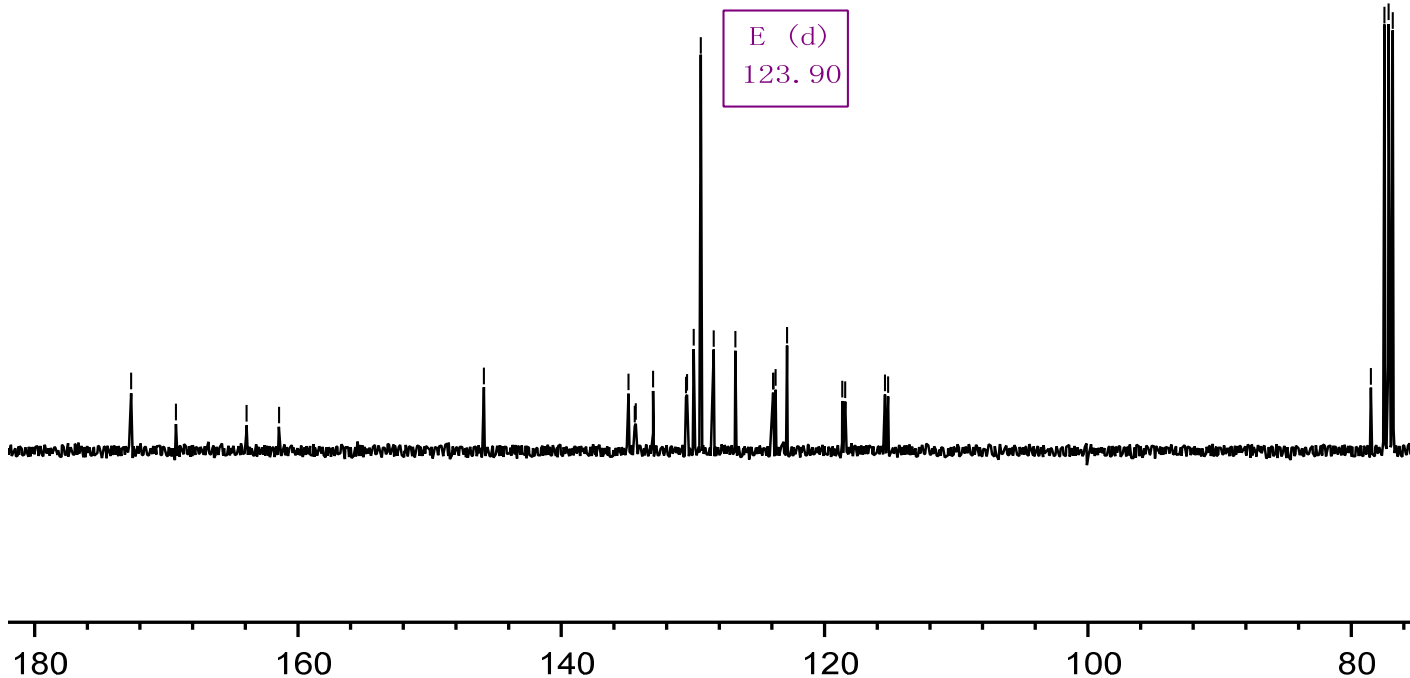

160

140

120

100

80

60

40

20 0 


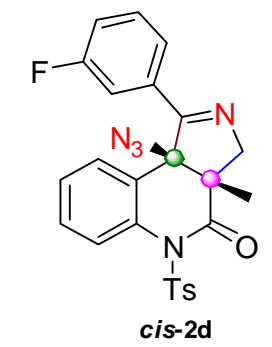

${ }^{19} \mathrm{~F} \mathrm{NMR}\left(\mathrm{CDCl}_{3}, 376 \mathrm{MHz}\right)$
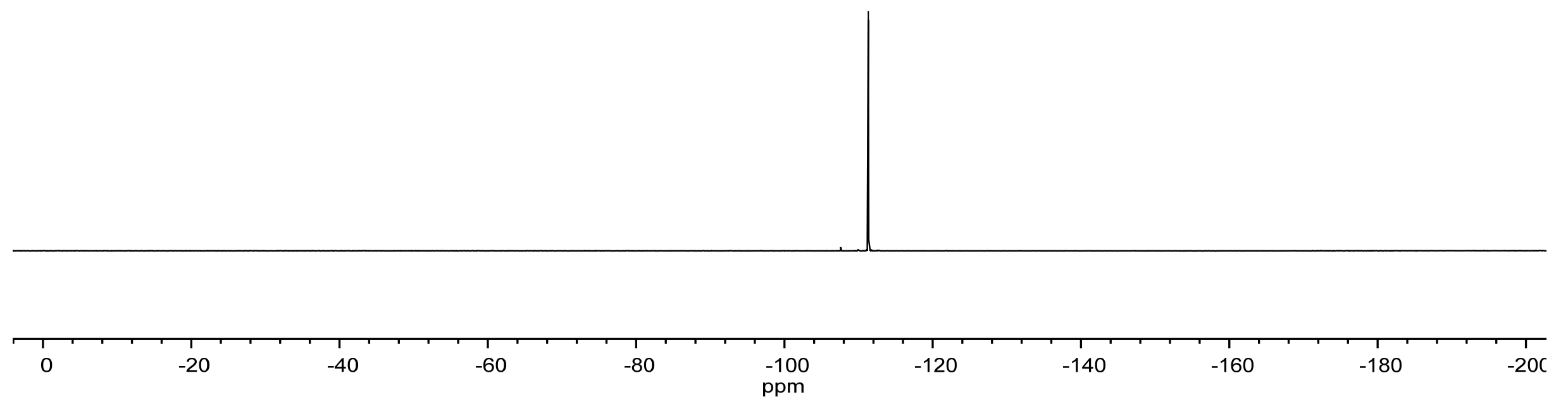
œ

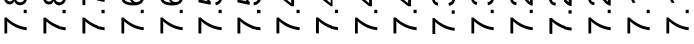

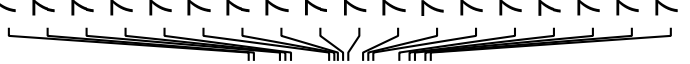
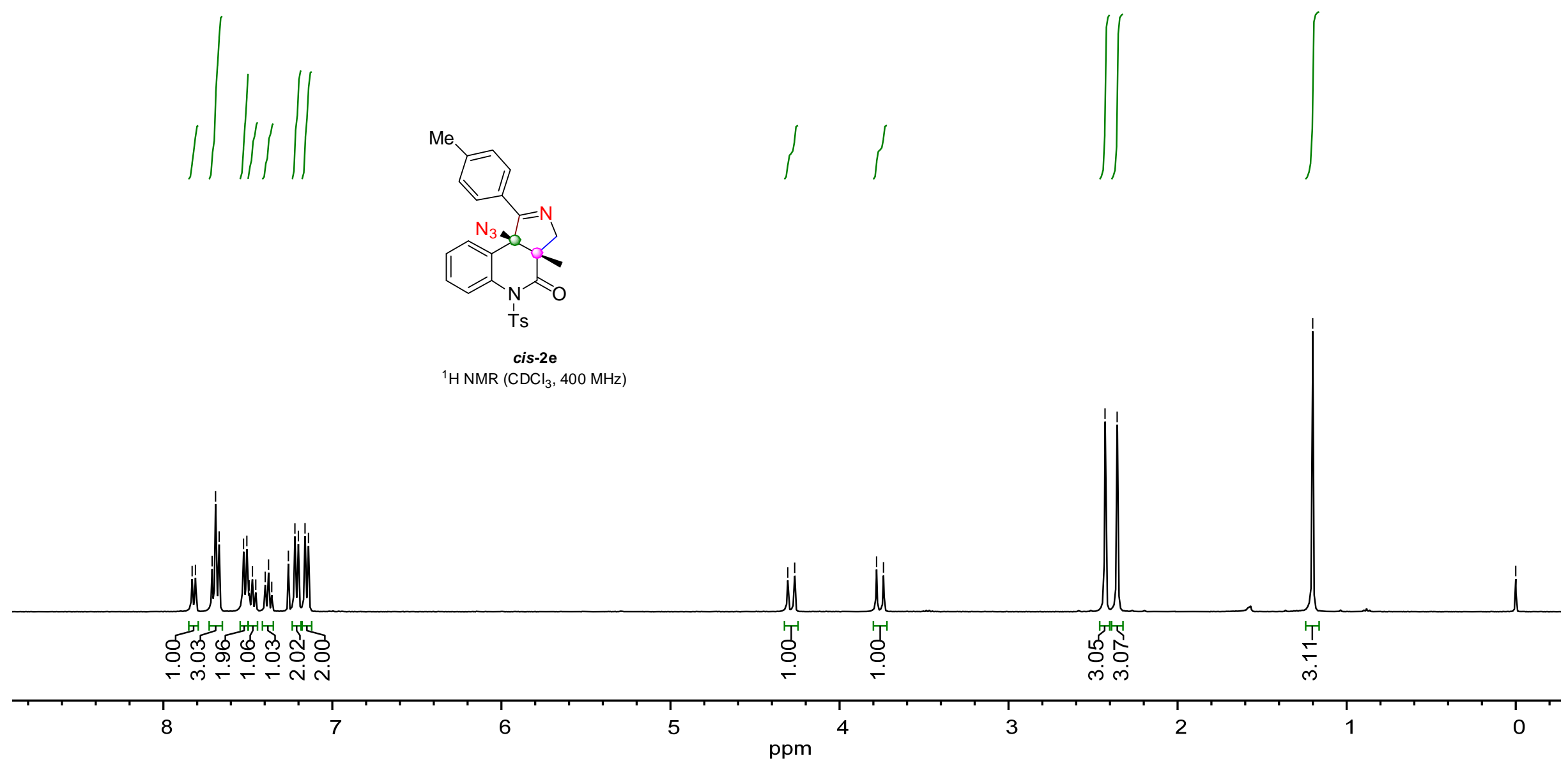

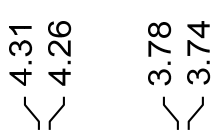

$\stackrel{m}{\forall} \underset{n}{n}$

i i

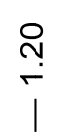

움 


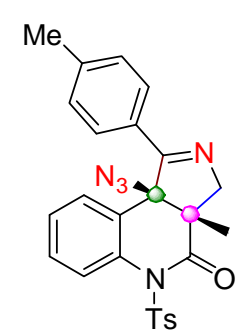

cis-2e

${ }^{13} \mathrm{C}$ NMR $\left(\mathrm{CDCl}_{3}, 100 \mathrm{MHz}\right)$
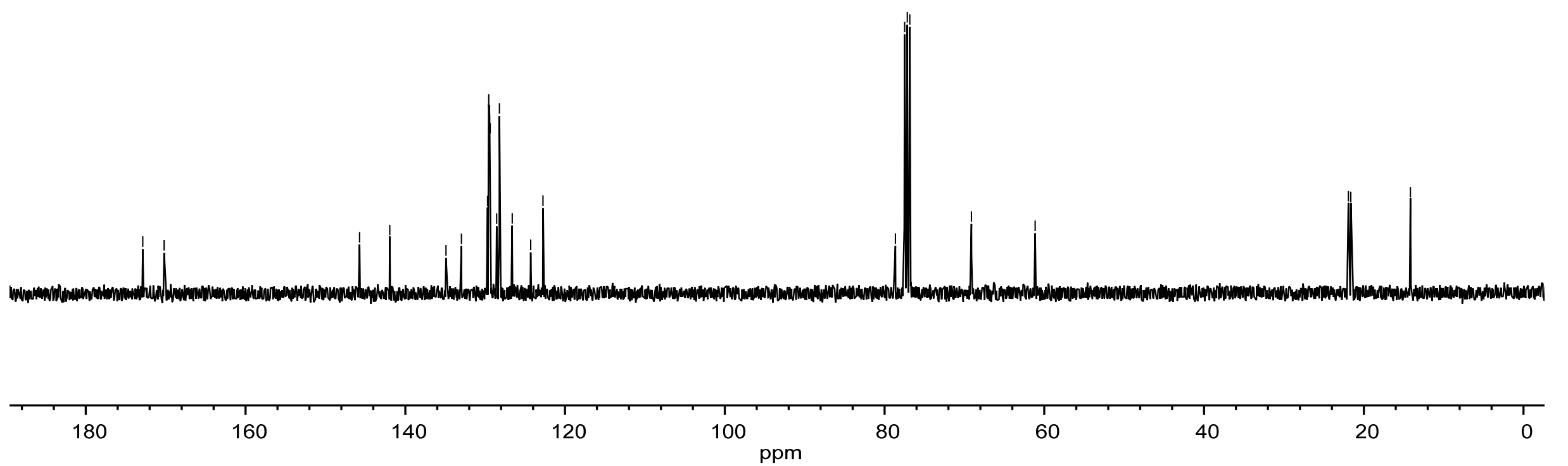

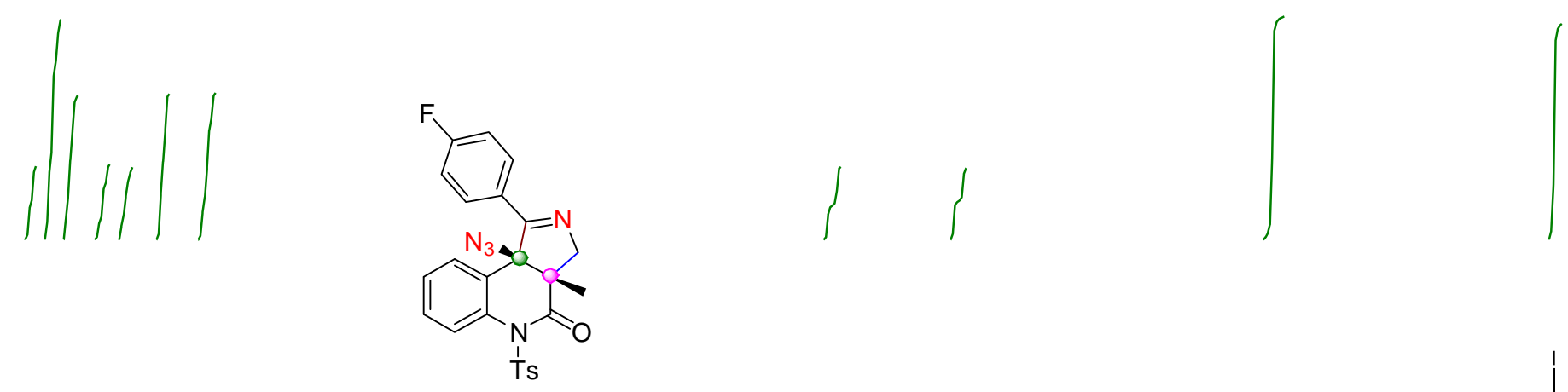

${ }^{1} \mathrm{H} \mathrm{NMR}\left(\mathrm{CDCl}_{3}, 400 \mathrm{MHz}\right)$

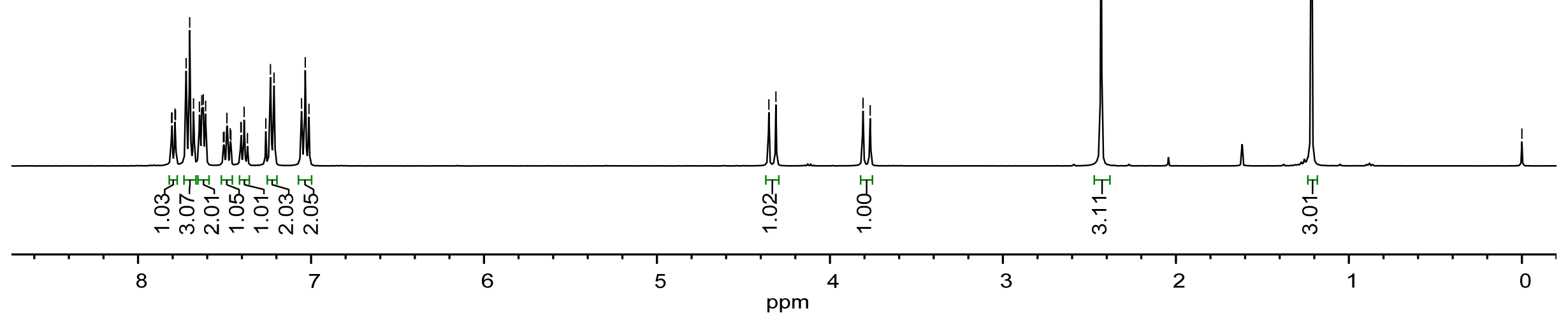




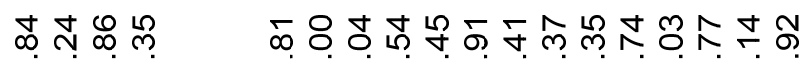

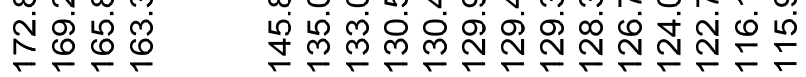

1111 近

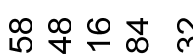

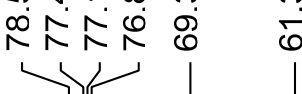

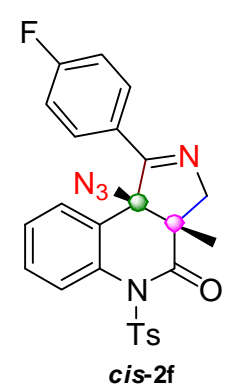

$\left.{ }^{13} \mathrm{C} \mathrm{NMR} \mathrm{(CDCl} 3,100 \mathrm{MHz}\right)$

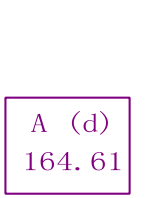

\begin{tabular}{|c|}
\hline D (d) \\
129.39 \\
\hline C $(d)$ \\
130.50 \\
\hline
\end{tabular}

\begin{tabular}{|c|}
\hline B (d) \\
116.03 \\
\hline
\end{tabular}

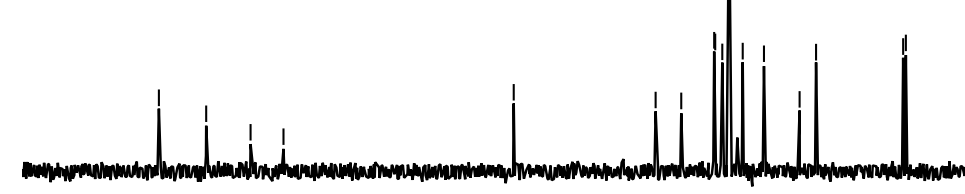

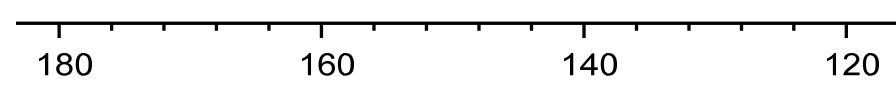

100

80

60

40

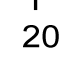



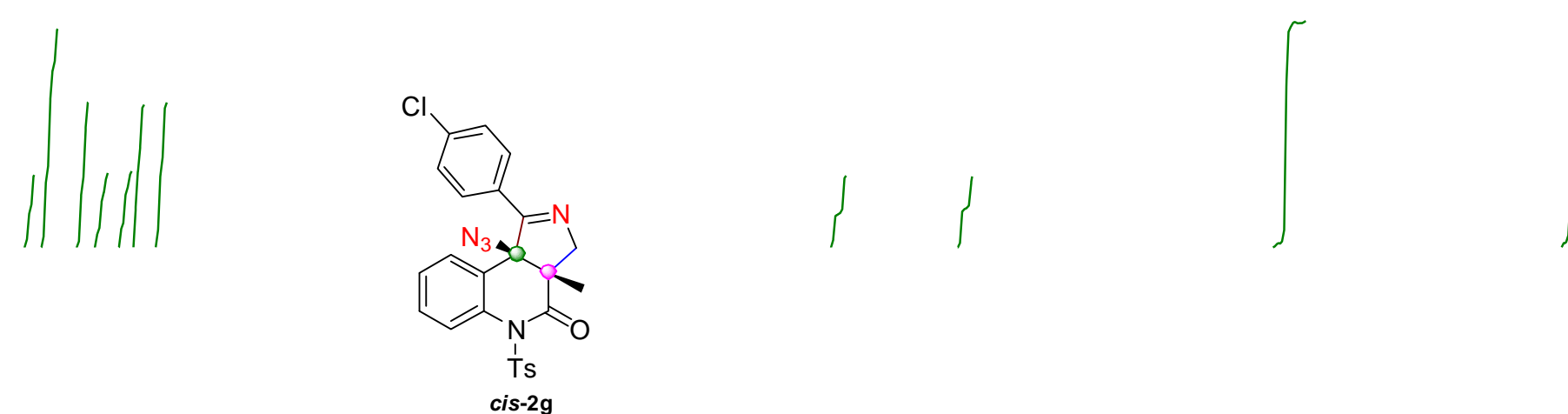

${ }^{1} \mathrm{H} \mathrm{NMR}\left(\mathrm{CDCl}_{3}, 400 \mathrm{MHz}\right)$

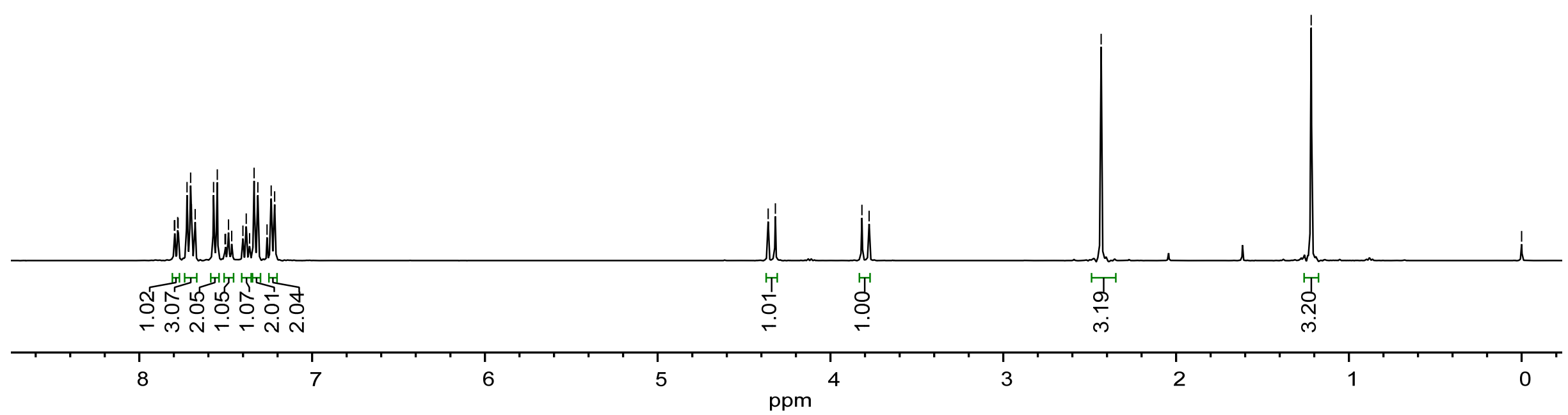




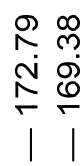

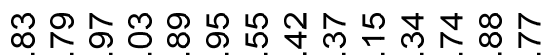

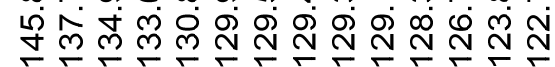

1

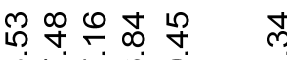

余余通

$\stackrel{\bar{\sigma}}{\stackrel{\infty}{\leftarrow}}$
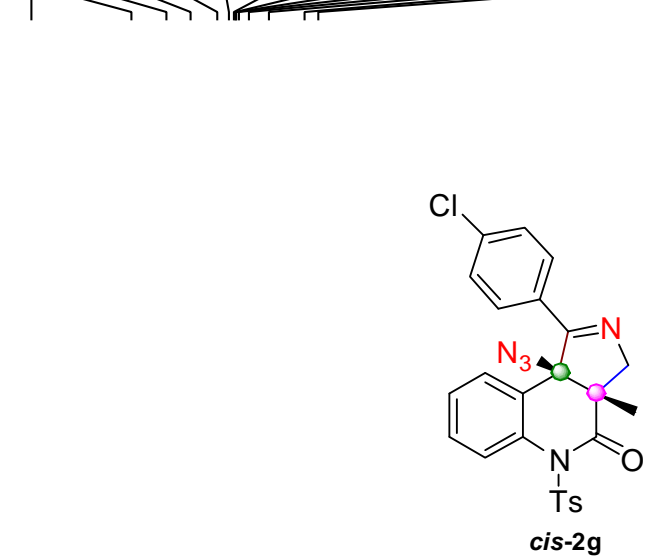

${ }^{13} \mathrm{C} \mathrm{NMR}\left(\mathrm{CDCl}_{3}, 100 \mathrm{MHz}\right)$
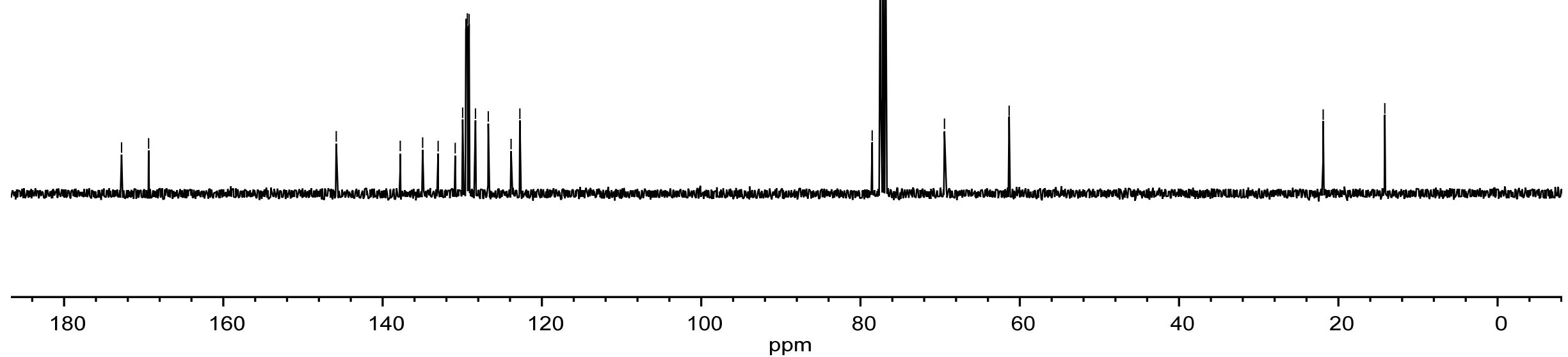


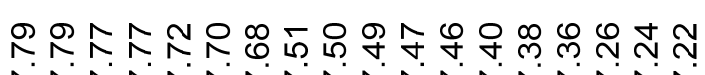

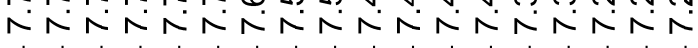

는

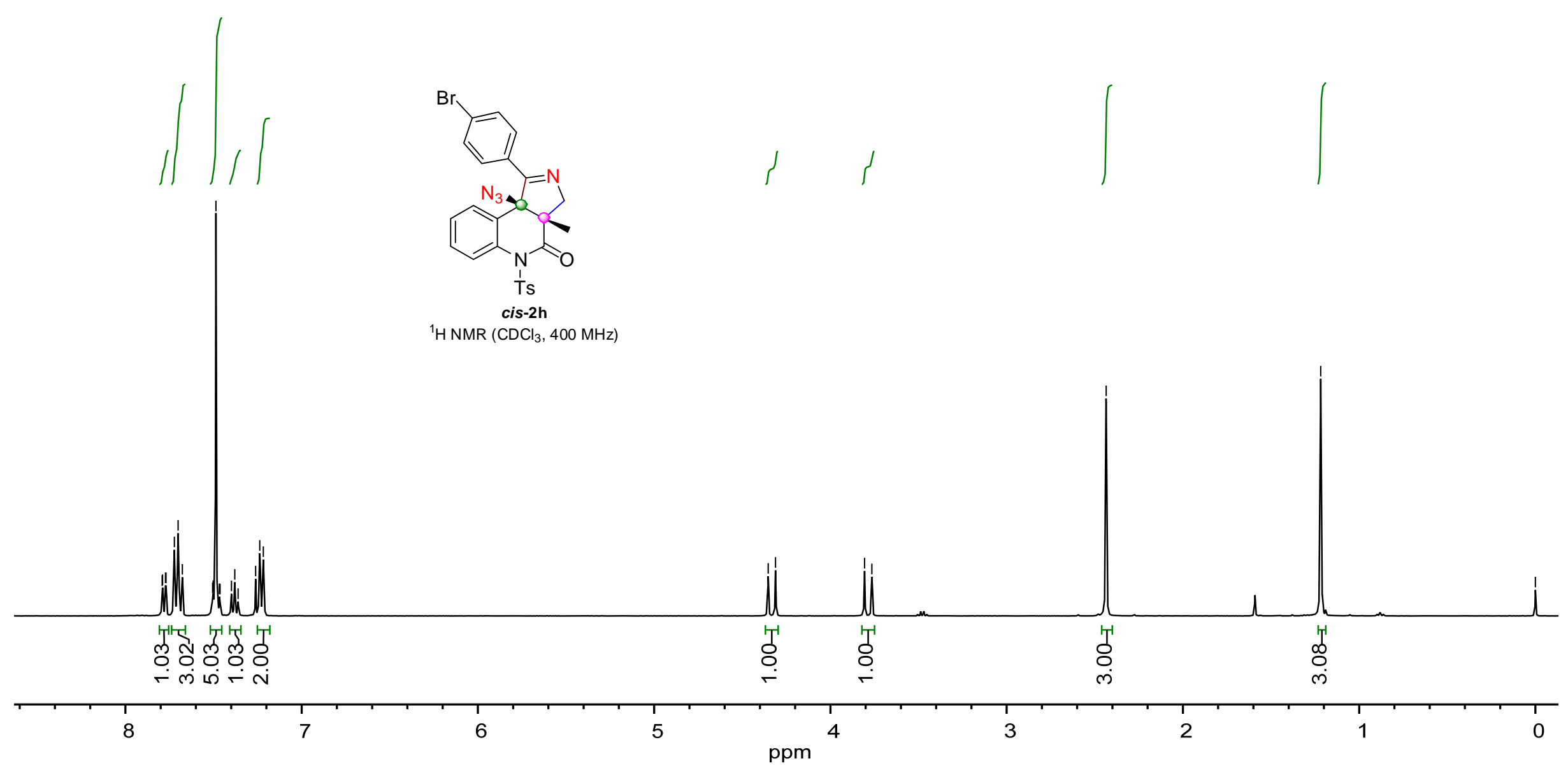

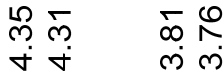

in

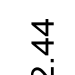

$\stackrel{N}{\stackrel{N}{+}}$

8

i 


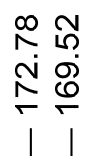

ஜ

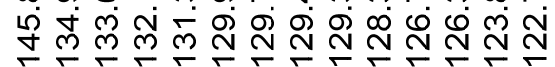

店

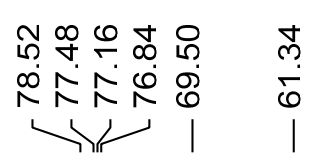

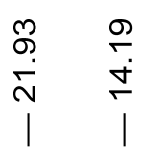

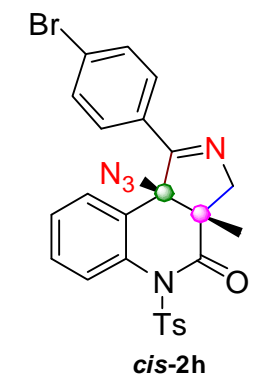

${ }^{13} \mathrm{C} \mathrm{NMR}\left(\mathrm{CDCl}_{3}, 100 \mathrm{MHz}\right)$
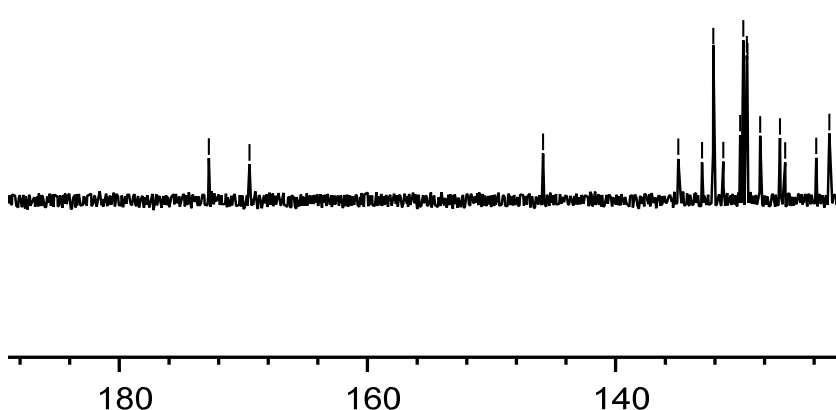

160

140

120

100

80

60

40

20 

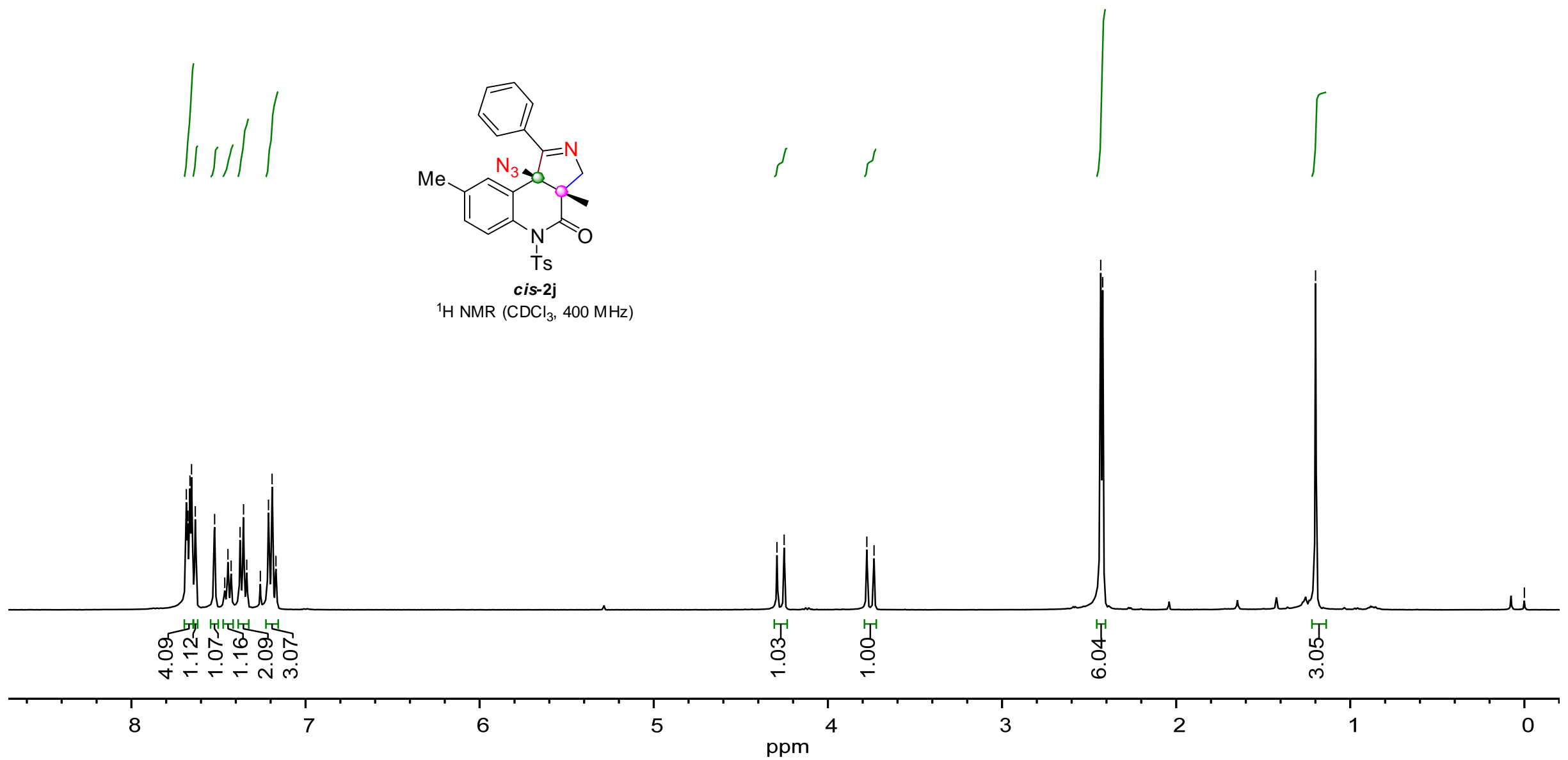


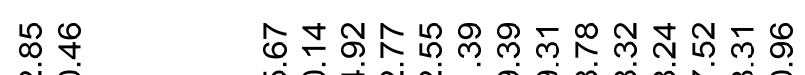

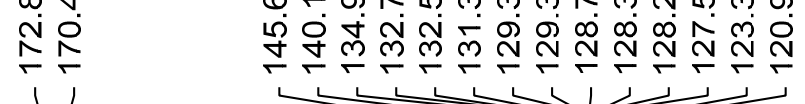

กิ่

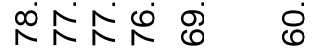

요ㅇㅛㅛ 웅

r

।

ते

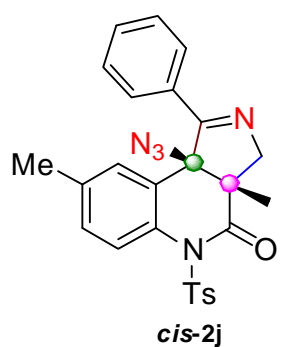

$\left.{ }^{13} \mathrm{C} \mathrm{NMR} \mathrm{(CDCl}, 100 \mathrm{MHz}\right)$
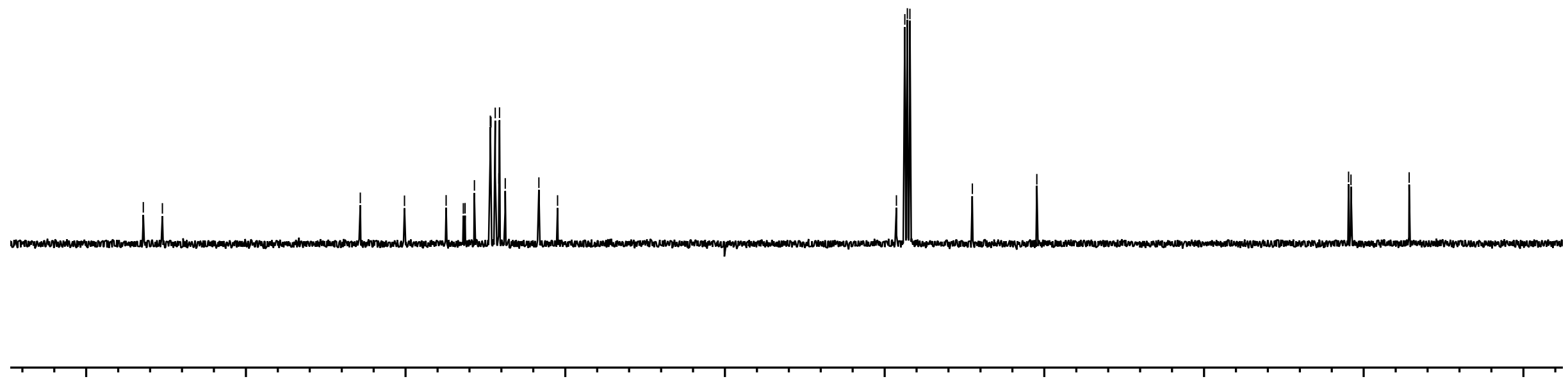

160

140

120

100

80

60 

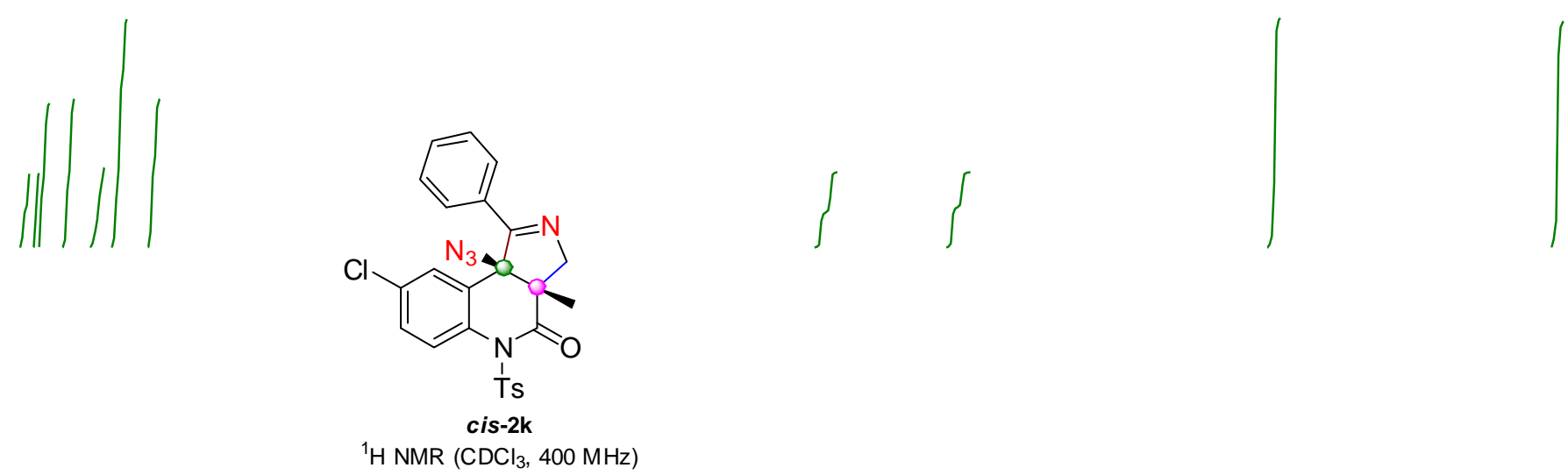

${ }_{1} \mathrm{H} \mathrm{NMR}\left(\mathrm{CDCl}_{3}, 400 \mathrm{MHz}\right)$

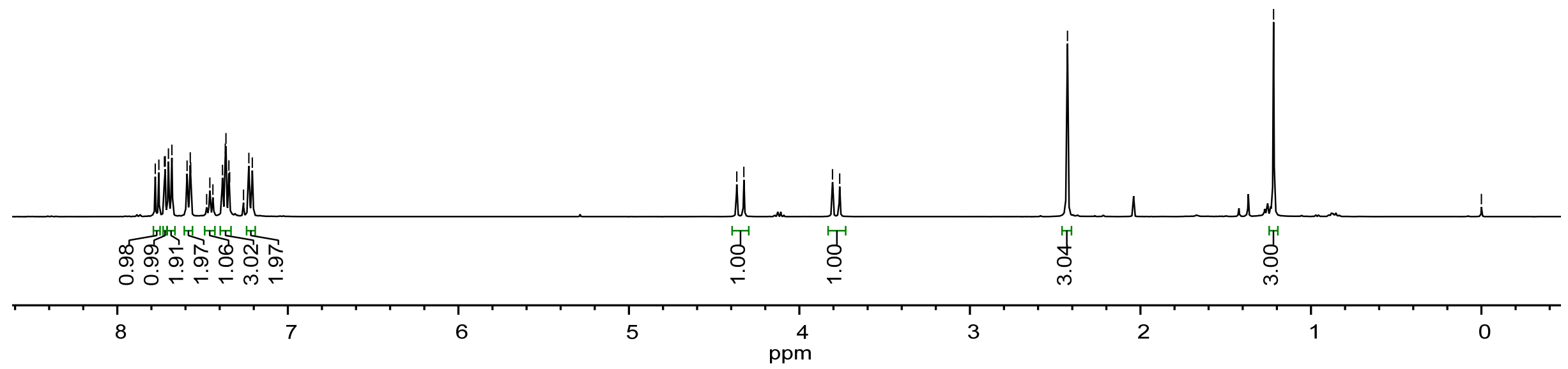




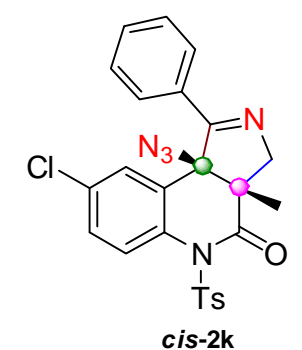

${ }^{13} \mathrm{C} \mathrm{NMR}\left(\mathrm{CDCl}_{3}, 100 \mathrm{MHz}\right)$
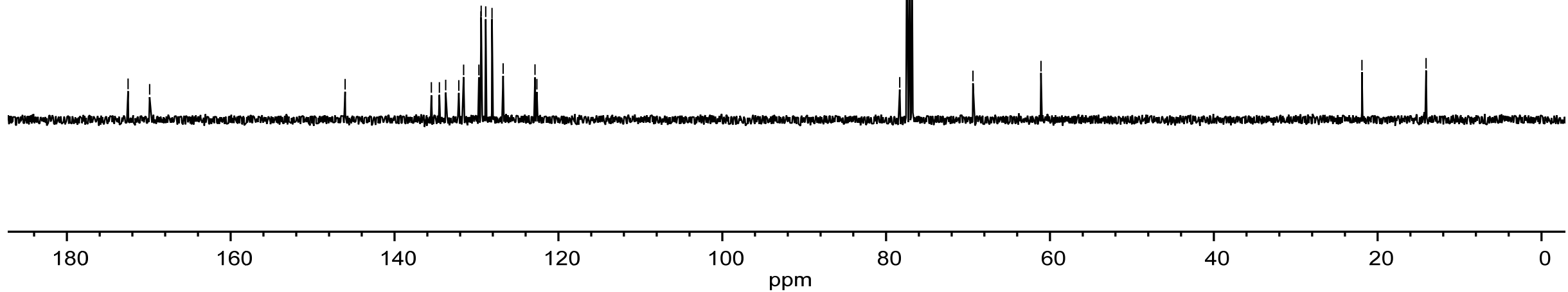
○

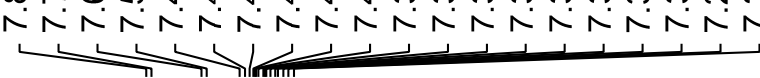

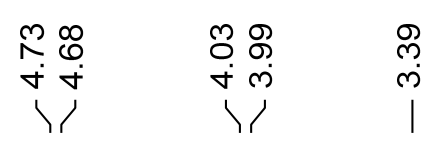
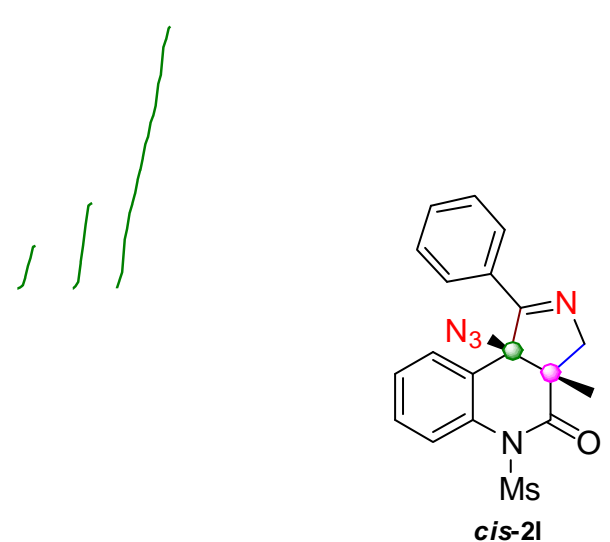

${ }^{1} \mathrm{H} \mathrm{NMR}\left(\mathrm{CDCl}_{3}, 400 \mathrm{MHz}\right)$

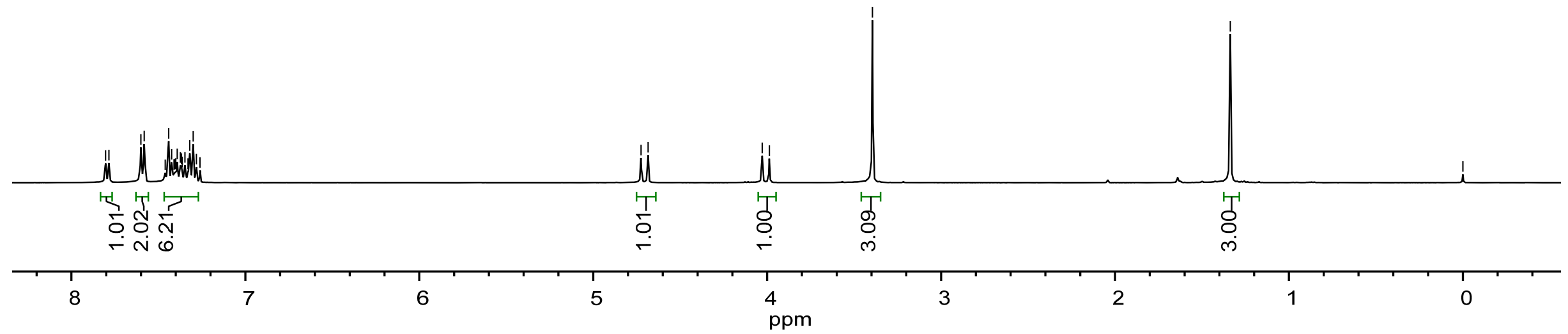




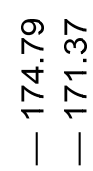

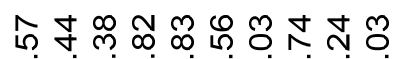
岱

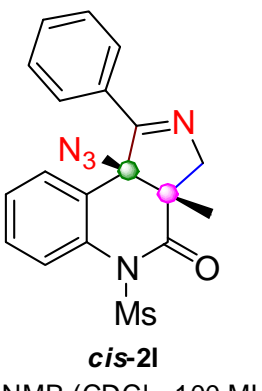

$\left.{ }^{13} \mathrm{C} \mathrm{NMR} \mathrm{(CDCl} 3,100 \mathrm{MHz}\right)$

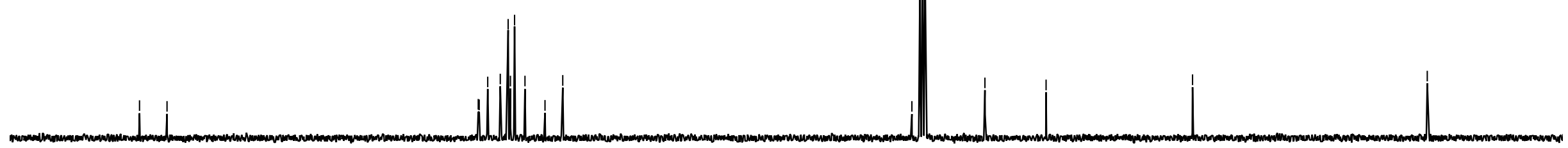

